\title{
Convex Programs for Minimal-Area Problems
}

Cite this article as: Matthew Headrick and Barton Zwiebach, Convex Programs for Minimal-Area Problems, Communications in Mathematical Physics https://doi.org/10.1007/s00220-020-03732-1

This Author Accepted Manuscript is a PDF file of an unedited peer-reviewed manuscript that has been accepted for publication but has not been copyedited or corrected. The official version of record that is published in the journal is kept up to date and so may therefore differ from this version.

Terms of use and reuse: academic research for non-commercial purposes, see here for full terms. https://www.springer.com/aam-terms-v1 


\section{Communications in Mathematical Physics Convex programs for minimal-area problems --Manuscript Draft--}

\section{Manuscript Number:}

Full Title:

Article Type:

Funding Information:
CIMP-D-19-00690R1

Convex programs for minimal-area problems

Original research

Office of Science

(DE-SC0009987)

Office of Science

(DE-SC0012567)

Dr. Matthew Headrick

Directorate for Mathematical and Physical

Sciences

(PHY-1053842)

Simons Foundation

(Simons Fellowship in Theoretical

Physics)

\begin{tabular}{|c|c|}
\hline Abstract: & $\begin{array}{l}\text { The minimal-area problem that defines string diagrams in closed string field } \\
\text { theory asks for the metric of least area on a Riemann surface with the condition that all } \\
\text { non-contractible closed curves have length at least } \$ 2 / p i \$ \text {. This is an extremal length } \\
\text { problem in conformal geometry as well as a problem in systolic geometry. We consider } \\
\text { the analogous minimal-area problem for homology classes of curves and, with the aid } \\
\text { of calibrations and the max flow-min cut theorem, formulate it as a local convex } \\
\text { program. We derive an equivalent dual program involving maximization of a concave } \\
\text { functional. These two programs give new insights into the form of the minimal-area } \\
\text { metric and are amenable to numerical solution. We explain how the homology } \\
\text { problem can be modified to provide the solution to the original homotopy problem. }\end{array}$ \\
\hline Corresponding Author: & $\begin{array}{l}\text { Matthew Headrick, Ph.D. } \\
\text { Brandeis University } \\
\text { Waltham, MA UNITED STATES }\end{array}$ \\
\hline \multicolumn{2}{|l|}{$\begin{array}{l}\text { Corresponding Author Secondary } \\
\text { Information: }\end{array}$} \\
\hline Corresponding Author's Institution: & Brandeis University \\
\hline \multicolumn{2}{|l|}{$\begin{array}{l}\text { Corresponding Author's Secondary } \\
\text { Institution: }\end{array}$} \\
\hline First Author: & Matthew Headrick, Ph.D. Rece ist : $\mathrm{Al}$ \\
\hline \multicolumn{2}{|l|}{ First Author Secondary Information: } \\
\hline \multirow[t]{2}{*}{ Order of Authors: } & Matthew Headrick, Ph.D. \\
\hline & Barton Zwiebach \\
\hline \multicolumn{2}{|c|}{ Order of Authors Secondary Information: } \\
\hline Author Comments: & $\begin{array}{l}\text { We are submitting this paper together with a companion paper, entitled "Minimal-area } \\
\text { metrics on the Swiss cross and punctured torus". These two papers are closely related; } \\
\text { in fact, to understand and appreciate the second one it is very useful to have read the } \\
\text { first one (and conversely reading the second one will help one understand and } \\
\text { appreciate the first one). We therefore suggest sending them to the same referee(s). }\end{array}$ \\
\hline Response to Reviewers: & $\begin{array}{l}\text { We thank the referees for their careful reading of our paper and thoughtful comments. } \\
\text { We are resubmitting the paper without revisions. }\end{array}$ \\
\hline
\end{tabular}


lick here to view linked References

\title{
Convex programs for minimal-area problems
}

\author{
Matthew Headrick \\ Martin Fisher School of Physics \\ Brandeis University \\ Waltham MA 02143, USA \\ headrick@brandeis.edu \\ and \\ Barton Zwiebach \\ Center for Theoretical Physics \\ Massachusetts Institute of Technology \\ Cambridge MA 02139, USA \\ zwiebach@mit.edu
}

\begin{abstract}
The minimal-area problem that defines string diagrams in closed string field theory asks for the metric of least area on a Riemann surface with the condition that all non-contractible closed curves have length at least $2 \pi$. This is an extremal length problem in conformal geometry as well as a problem in systolic geometry. We consider the analogous minimal-area problem for homology classes of curves and, with the aid of calibrations and the max flow-min cut theorem, formulate it as a local convex program. We derive an equivalent dual program involving maximization of a concave functional. These two programs give new insights into the form of the minimal-area metric and are amenable to numerical solution. We explain how the homology problem can be modified to provide the solution to the original homotopy problem.
\end{abstract}




\section{Contents}

1 Introduction and summary $\quad 2$

2 Review of closed string field theory and minimal-area metrics $\quad 6$

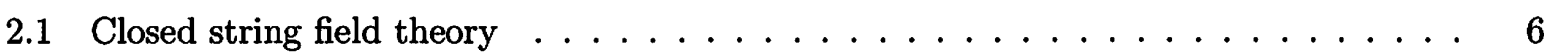

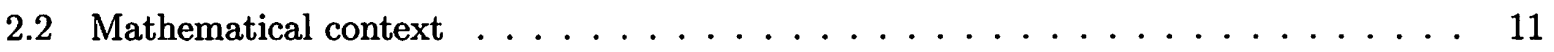

3 Review of convex programming and max flow-min cut $\quad 12$

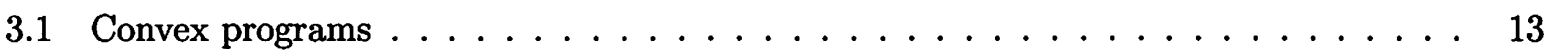

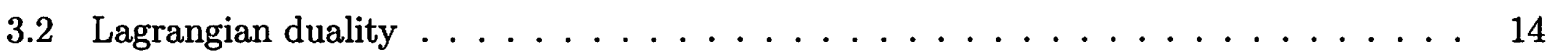

3.3 Calibrations and the $\max$ flow-min cut theorem $\ldots \ldots \ldots \ldots \ldots$

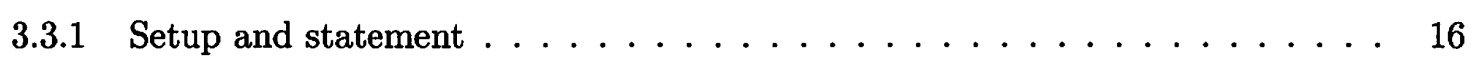

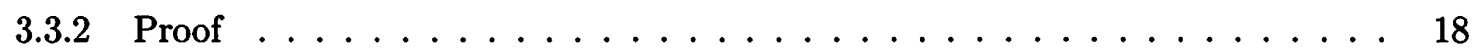

4 The homotopy and homology problems $\quad 22$

4.1 Notation . . . . . . . . . . . . . . . . . . . . . . . . . 23

4.2 Homotopic minimal-area programs $\ldots \ldots \ldots \ldots \ldots \ldots \ldots$

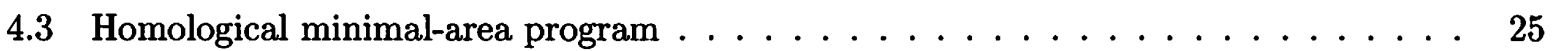

4.4 Reformulation as a local problem using calibrations . . . . . . . . . . . . 27

5 An equivalent dual problem $\quad 30$

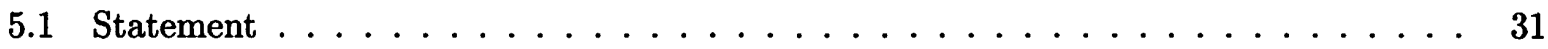

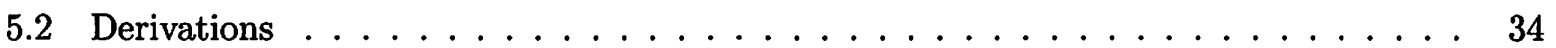

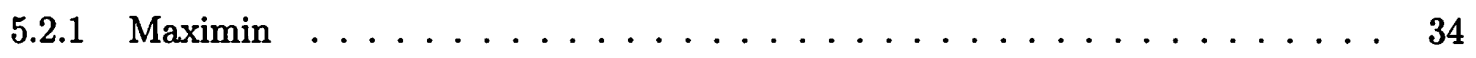

5.2 .2 From homology MAP $\ldots \ldots \ldots \ldots \ldots \ldots \ldots \ldots \ldots \ldots$

5.2 .3 From primal MAP $\ldots \ldots \ldots \ldots \ldots \ldots \ldots \ldots$

6 Simple examples $\quad 41$

6.1 Cylinder . . . . . . . . . . . . . . . . . . . . . 41

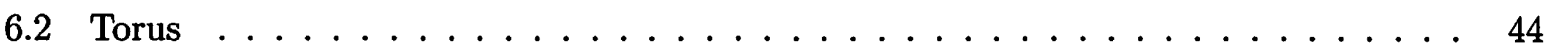

7 Properties of the minimal-area metric 46

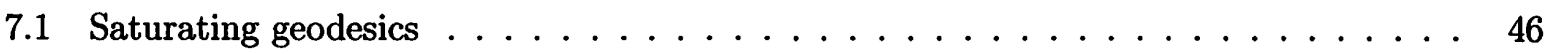

7.2 The metric and the primal and dual solutions $\ldots \ldots \ldots \ldots \ldots \ldots$

7.3 Normal coordinates for bands of geodesics . . . . . . . . . . . . . . 49

7.4 Properties of the solution from the dual $\ldots \ldots \ldots \ldots \ldots$

7.4 .1 Segregation of level sets $\ldots \ldots \ldots \ldots \ldots \ldots$

7.4 .2 Cylinder versus pants $\ldots \ldots \ldots \ldots \ldots \ldots \ldots$

7.5 Quadratic differentials and the dual objective $\ldots \ldots \ldots \ldots \ldots \ldots$

8 Back to the homotopy problem $\quad 59$

8.1 The homotopy problem reduced to homology . . . . . . . . . . . . . . . 59

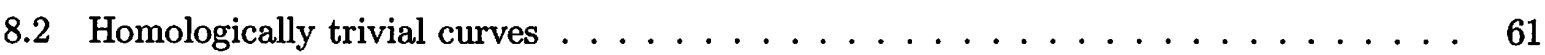

8.2 .1 Defining a suitable covering space $\ldots \ldots \ldots \ldots \ldots \ldots \ldots$ 
8.2 .2 Defining the calibration on $\tilde{M} \ldots \ldots \ldots \ldots \ldots \ldots$

8.2 .3 Analysis of curves on $\tilde{M} \ldots \ldots \ldots \ldots \ldots \ldots$

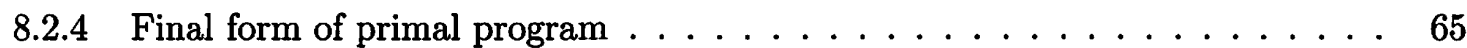

A Notation and some useful formulae

\section{Introduction and summary}

Progress in our mastery of string theory often requires finding answers to certain mathematical questions. This was the situation with string field theory, whose formulation needs concrete ways of constructing the moduli spaces of Riemann surfaces with marked points, and finding a way to assign local complex coordinates at the marked points for each surface in each moduli space [1-3].

While the formulation of open string field theory required some results on the moduli spaces of Riemann surfaces with boundaries, most of the needed facts were known in the literature through work of Strebel [4]. The case of closed string field theory proved much more challenging. After some work elucidating the construction of the moduli spaces of spheres with marked points $[5,6]-\mathrm{a}$ construction relevant to classical closed string field theory-the general construction of all moduli spaces required for the full quantum action was claimed to arise via the following minimal-area problem $[7,8]$ :

Minimal-Area Problem: Given a genus $g$ Riemann surface with $n \geq 0$ marked points ( $n \geq 2$ for $g=0$ ), find the metric of minimal (reduced) area under the condition that the length of any noncontractible closed curve be greater than or equal to $2 \pi$.

The marked points are the locations where string vertex operators are inserted. The metric's Weyl class is fixed by the complex structure: the length element is $d s=\rho|d z|$, where $z=x+i y$ is a complex coordinate, and the area element is $d A=\rho^{2} d x \wedge d y$. The homotopy of curves is relative to the punctures, and therefore curves surrounding each puncture are considered noncontractible. All noncontractible closed curves are required to have length at least $2 \pi$. That number, while convenient for closed string theory, is just conventional; it could be set equal to 1 or to any other number. In the extremal metric, that number happens to be the systole of the surface, that is, the length of the shortest noncontractible closed geodesic. For $g \geq 1$ and $n=0$, the area of the extremal metric is finite. When $n \geq 1$, however, one finds that in some open neighborhood of each of the punctures the extremal metric is a semi-infinite cylinder of circumference $2 \pi$. As a result, the area is infinite and one must consider a regularized "reduced area" for minimization [8]. Briefly stated, the above minimal-area problem gives a string field theory because the plumbing of extremal metrics gives extremal metrics.

For a minimal-area problem where the length condition is applied to a set of homotopy classes of curves that have non-intersecting representatives, the extremal metric is known and arises as the norm of a Jenkins-Strebel quadratic differential [4]. In our problem, we constrain all homotopy classes of curves. It turns out that for genus-zero surfaces with marked points, the extremal metric nonetheless arises from quadratic differentials. This is not the case, however, for large classes of surfaces in each of the moduli spaces of $g \geq 1$ Riemann surfaces. Generically, the extremal metric does not arise from a quadratic differential, there is no general method to find it and, in fact, the 
solution is unknown. The length constraint, which applies to an infinite set of curves, makes even a numerical analysis of the problem very challenging.

The minimal-area problem is a particularly hard case of the problem of finding the extremal length conformal invariant [9]. Moreover, the problem can also be considered as one in systolic geometry [10-12]. Gromov [13] studied in detail the problem of finding the minimal-volume Riemannian metric on an $n$-dimensional manifold $M$ with a given systole. The specialization of this question to two-dimensional manifolds with fixed complex structure $[13,14]$ is the same problem posed above. For two-dimensional manifolds but without fixing the Weyl class of the metric, a set of results was derived by Calabi [15]. The case of metrics that are, additionally, of non-positive curvature is also of interest [16].

The extremal metric for our minimal-area problem is expected to have systole-length geodesics that cover the surface. For brevity we will use the term systolic geodesic for a systole-length geodesic. Near the marked points these geodesics are circles foliating a flat semi-infinite cylinder, with the marked point at infinite distance. Through each point on that cylinder there is just one systolic geodesic. On the rest of the surface there can exist regions with one and only one systolic geodesic going through each point, but also regions where more than one such geodesic goes through each point. In general the surface is covered by multiple bands of intersecting and non-intersecting systolic geodesics. The metric is not known as soon as systolic bands intersect. This will happen for surfaces over finite subsets of each moduli space $\mathcal{M}_{g, n}$ of Riemann surfaces of genus $g \geq 1$ with $n$ marked points.

This paper reports on progress on this minimal-area problem. The advances rely, to a large degree, on the geometrical application of tools from the subject of convex optimization [18]. A basic example of such an application, which we will use extensively in this paper, is the max flow-min cut theorem on manifolds, which is proved with the help of strong duality of convex programs [19]. A review of this material can be found in the recent paper [20] of Hubeny and Headrick. (See also [21] for a different physical application of the max flow-min cut theorem on manifolds, in the context of holographic entanglement entropy.)

To make progress on the minimal-area problem, we begin by modifying it. As stated, the length constraints apply to homotopy classes of curves. Instead, we consider length constraints applied to homology classes of curves. Any closed curve that is homologically nontrivial is also homotopically nontrivial, but there are homotopically nontrivial curves that are homologically trivial. The length of those curves are not constrained in the homology problem. These curves, however, can be dealt with by passing to a suitable covering space where they become homologically nontrivial. We discuss in detail how a suitably extended homology problem provides a solution of the original minimal-area problem.

In the general homology minimal-area problem, we fix a set $\left\{C_{\alpha}\right\}$ of homology cycles and numbers $\left\{\ell_{\alpha}\right\}$ and require the length of every curve in the class $C_{\alpha}$ to have length at least $\ell_{\alpha}$. In this paper we present two main advances on this problem:

1. The first advance is to replace the length constraints by the existence of calibrations: closed one-forms with norm everywhere less than or equal to one. A calibration with period $\ell_{\alpha}$ on a homology cycle $C_{\alpha}$ constrains the lengths of all curves in the corresponding homology class to be greater than or equal to $\ell_{\alpha}$. Requiring the existence of a calibration places no additional constraints because the max flow-min cut theorem guarantees the converse: if all curves in the 
homology class have length at least $\ell_{\alpha}$ then there is a calibration with period $\ell_{\alpha}$. The length constraint, a nonlocal condition on the metric on the surface, is implemented as a local condition on the one-form calibration, a significant simplification. The calibrations can be written in terms of abelian differentials consistent with the requisite periods plus exact one-forms. Minimizing the area subject to the calibration constraint is a convex optimization problem, or convex program, henceforth called the primal program. This immediately guarantees that any local minimum is a global minimum (i.e. there are no false minima). Furthermore, it allows the application of powerful numerical methods for solving convex programs.

2. The second advance follows from applying Lagrangian duality to the primal. This means adding Lagrange multipliers to impose the constraints and then solving for the original variables of the primal. The result is a dual program in which we maximize a functional of the Lagrange multipliers. The dual program variables are a collection of functions $\varphi^{\alpha}$ and numbers $\nu^{\alpha}$ associated with the homology classes $C_{\alpha}$. The value of $\nu^{\alpha}$ is the discontinuity of $\varphi^{\alpha}$ across an arbitrarily chosen representative of the class $C_{\alpha}$. At the optimum, the curves of constant $\varphi^{\alpha}$ are the systolic geodesics in the class $C_{\alpha}$ and $\nu^{\alpha}$ is, roughly, the height of the annular domain covered by those geodesics. Because of convexity of the primal and an easily satisfied technical condition, the property of strong duality holds: the minimum in the primal is guaranteed to coincide with the maximum of the dual.

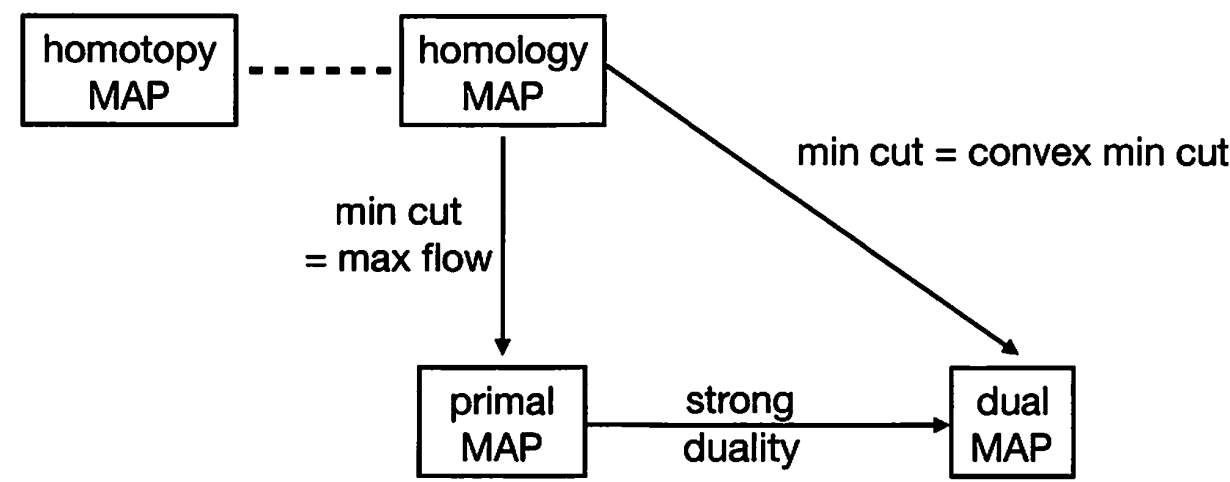

Figure 1: Relations among the minimal-area programs (MAPs) discussed in this paper. The homotopy MAP is given in (4.14), the homology MAP in (4.15), the primal MAP in (4.32), and the dual program in (5.7). The dotted line indicates that the programs are related but not equivalent. The arrows relate equivalent programs, and the text on the arrows indicate the main tool used to prove the equivalence.

The relations between the various programs are illustrated in Figure 1. The homotopy minimal area problem (MAP) suggests the analogous homology problem, the two corresponding boxes joined by a dotted line. Our work with covering spaces in section 8 shows how to use the homology version of the problem to solve the closed string field theory (homotopy) problem. The passage from homology MAP to primal MAP was explained in item 1 above, and the dualization in item 2. An alternative derivation, shown by the diagonal arrow in the diagram, provides additional insight into the dual and the max flow-min cut theorem.

In an accompanying paper [22], we illustrate the programs described here by numerically solving for the unknown extremal metric of a relatively simple Riemann surface, the square torus with one 
boundary. The extremal metric on this surface is also the extremal metric on the so-called Swiss cross. The extremal metric displays crossing bands of geodesics and non-zero Gaussian curvature. We also find the previously unknown metric for the once-punctured square torus. This metric is needed for the definition of closed string field theory to one loop. We will demonstrate that the programs are easily solved numerically. Having a minimization program and a maximization program with the same solution proves very helpful. The methods of the present paper can also be applied to the study of conformal metrics of non-positive curvature on Riemann surfaces. These metrics are also of interest for string field theory and exhibit surprising features revealed by the numerical study: not all of the Riemann surface is necessarily covered by saturating geodesics $[23]^{1}$.

In addition to being readily amenable to numerical solution, the programs above offer novel theoretical insights into the minimal-area metrics. Indeed, strong duality implies complementary slackness, which relates the two programs, giving information about the solution. In particular:

(i) We find a remarkably simple relation, (7.8), between the extremal area $A$ of the surface, the heights $\nu^{\alpha}$ of geodesic bands, and the minimum lengths $\ell_{\alpha}$ :

$$
A=\sum_{\alpha} \nu^{\alpha} \ell_{\alpha}
$$

Thus, even when bands cross and there may be curvature, the total area of the surface is the sum of that of flat cylinders of height $\nu^{\alpha}$ and circumference $\ell_{\alpha}$.

(ii) The dual program allows the definition of the local density $\rho_{\alpha}$ of geodesics in the $\alpha$-band at any point on the surface. We find that the pointwise sum of the densities of all the bands is constant over the entire surface, (7.23). Equivalently, a sum rule constrains the metric at every point of the surface $((7.11)$ and (7.21)). The sum rule implies that a region foliated by a single band of systole-length geodesics must be flat, a fact previously established by a more involved argument $[25,26]$.

We have tried to understand in what way the minimal-area metrics generalize those that arise as the norm of Jenkins-Strebel quadratic differentials. We have also tried to develop intuition about the somewhat mysterious dual program. Here are a few remarks:

(a) The systolic geodesics in the extremal metrics are the generalization of the horizontal trajectories of these quadratic differentials. The heights $\nu^{\alpha}$ of the dual program generalize the heights of the ring domains in the quadratic differentials. It is striking that in the systolic problem the area formula (1.1) takes exactly the same form as for quadratic differentials, where the metric is indeed that defining flat cylinders of prescribed circumference.

(b) When constraining homotopy classes of curves with representatives that do not intersect, quadratic differentials arise as the problem of maximizing the weighted sum $\sum \ell_{\alpha}^{2} M_{\alpha}$ where $M_{\alpha}$ are the moduli of non-overlapping ring domains $R_{\alpha}$ in the homotopy classes constrained with $\ell_{\alpha}$. We argue that the dual functional is a generalization of such a weighted sum. For this we see that the curves of constant $\varphi^{\alpha}$ (the precursors of systolic geodesics) segregate and form ring domains (subsection 7.4). Moreover, the dual program applied to a ring domain gives precisely $\ell_{\alpha}^{2} M_{\alpha}$ as the optimum.

\footnotetext{
${ }^{1}$ We have learned of work by Katz and Sabourau [24], dealing with systolic geometry of surfaces with Riemannian metrics of non-positive curvature. Regions without systolic geodesics play an important role in this work.
} 
This paper is organized as follows. In the section 2 we go over the basics of the minimal-area problem and its relevance to closed string field theory. In section 3 we begin by giving a brief review of the relevant aspects of convex optimization. We then examine and discuss in detail the max flow-min cut theorem, giving a proof based on duality that brings to the fore a "convex min cut" program that arises by convex relaxation. In section 4 , we introduce the homology minimalarea problem and reformulate it in terms of calibrations. The dual minimal-area program, given in various equivalent forms, is the subject of section 5 . We derive it by several routes, including directly from the convex min-cut program and by direct dualization of the primal. In section 6 we work through the solvable examples of the cylinder and torus to give some intuition for the primal and dual programs. In section 7 we use both programs to learn about the extremal metrics, and then turn to the dual, focusing on its properties and its relation to quadratic differentials. Finally, in section 8 we return to the homotopy problem that motivated this paper and show how the use of certain covering spaces makes it possible to solve the homotopy problem in terms of the homology problem.

\section{Review of closed string field theory and minimal-area metrics}

String perturbation theory constructs scattering amplitudes by integration of conformal field theory (CFT) correlators of vertex operators over moduli spaces of Riemann surfaces. Any construction of a closed string field theory must find a way to generate the moduli spaces of Riemann surfaces. More precisely, it must generate the moduli spaces $\mathcal{M}_{g, n}$ of Riemann surfaces of genus $g$ with $n$ marked points. The marked points, or punctures, are the places where vertex operators are inserted.

In this section we review the basic ideas that demonstrate that the minimal area problem stated in the introduction allows for the formulation of a closed string field theory. We also discuss how the minimal area problem fits in the context of conformal geometry and systolic geometry.

\subsection{Closed string field theory}

The classical closed string field theory must generate the genus zero moduli spaces $\mathcal{M}_{0, n}$ with $n \geq 3$. The full quantum theory must also generate the $g \geq 1$ moduli spaces with $n \geq 1$, although the case $n=0$ is also of interest. Similar remarks apply for open string field theory and open-closed string field theory in which the Riemann surfaces can also have boundary components and marked points on those boundaries. The string field theory generates the moduli spaces of Riemann surfaces via its Feynman diagrams, or string diagrams. The Feynman rules effectively become a prescription for building Riemann surfaces algorithmically and must construct each surface exactly once, thus generating the relevant moduli spaces. The Feynman rules use vertices and propagators.

As the string field theory builds surfaces it must also provide a local coordinate around each marked point. Such a coordinate is needed to insert vertex operators that are not dimension-zero primaries, as required for off-shell amplitudes. It turns out that the local coordinate need only be defined up to an overall phase.

In summary, the string field theory must provide:

1. A construction of the moduli spaces $\mathcal{M}_{g, n}$ of Riemann surfaces. 
2. For each surface, a choice of local coordinates (up to phases) at the marked points. The choice must be continuous over the moduli space.

3. The construction of the moduli spaces must be generated through Feynman rules, using vertices and propagators.

In order to satisfy such requirements canonically, an organizing principle is needed. In light-cone string field theory, for example, the string diagrams are the solution to the following problem [27]: Given a Riemann surface find the (unique) abelian differential with purely imaginary periods. This abelian differential provides a metric on the Riemann surface and allows one to visualize the surface concretely. The result is the familiar light-cone string diagrams.

For covariant closed string field theory the minimal-area problem stated in the introduction provides the organizing principle [8]. One could say closed string field theory raises that minimalarea problem. Since the minimal-area metric is unique, if known for all surfaces, it provides a concrete construction of the moduli spaces of Riemann surfaces. This is requirement (1) above.

In this minimal-area problem, the homotopy of closed curves is defined relative to the marked points, or punctures. Curves surrounding marked points are homotopically nontrivial and must satisfy the length condition. This, in fact, guarantees that (if the minimal-area metric exists) there is a neighborhood of the marked point that is isometric to a flat semi-infinite cylinder of circumference equal to the systole. ${ }^{2}$ This can be used to define a local coordinate $z$ around each marked point: one simply defines the last systolic geodesic on the cylinder to be the curve $|z|=1$ (see Figure 2). With $z=0$ the location of the marked point, the Riemann mapping theorem guarantees that there is a map from the coordinate disk $|z| \leq 1$ to the semi-infinite cylinder, defined uniquely up to a phase. This is requirement (2) above.
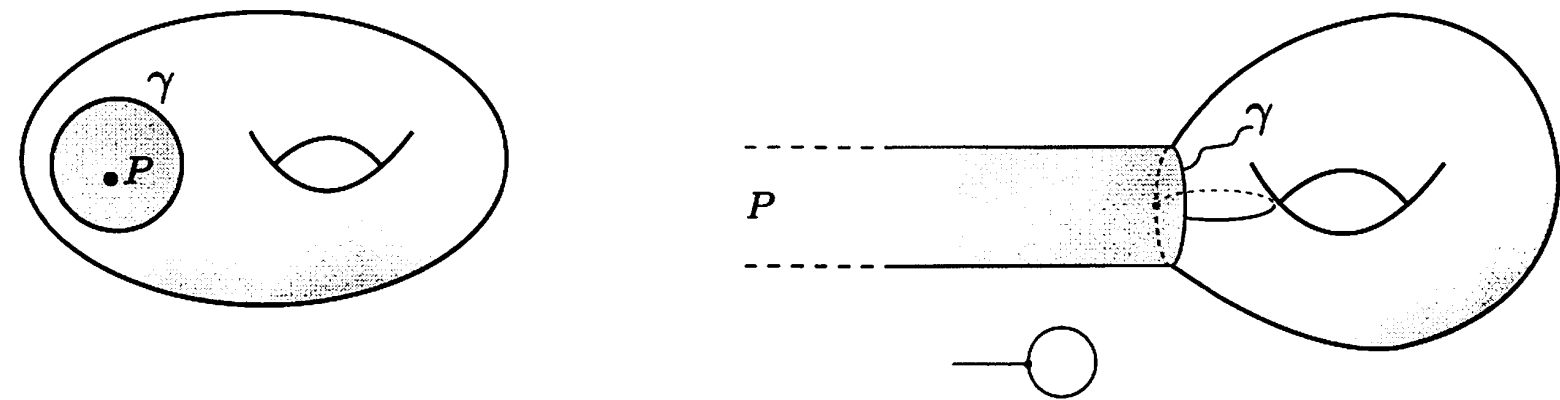

Figure 2: A torus with a marked point $P$ (left) and the same surface equipped with its minimal-area metric (right). The curve $\gamma$ is the last systolic geodesic on the semi-infinite cylinder. That curve is identified as the $|z|=1$ curve of a local coordinate that vanishes at $P$. In this way the minimal area metric fixes a local coordinate at the marked point. Once the surface is equipped with a minimal-area metric, the Feynman graph that would produce it is manifest: in this case the surface arises as a one-loop tadpole.

The most nontrivial constraint is in fact (3). Minimal area metrics, however, are naturally built with the use of vertices and propagators thus satisfying this requirement. Indeed, once a surface is equipped with its minimal area metric the associated Feynman graph that would produce it is

\footnotetext{
${ }^{2}$ The infinite area requires the definition of a finite regularized reduced area obtained with a subtraction that uses an arbitrary choice of local coordinates at the marked points.
} 
obvious (see Figure 2, right). The propagator can be viewed as a sewing prescription. Two local coordinates $z_{1}, z_{2}$ defined around two marked points (on two surfaces or on the same surface) allow sewing via $z_{1} z_{2}=t$ with $t$ a complex number $|t| \leq 1$. As indicated in Figure 3 the effect of sewing minimal area metrics with some fixed $t$ is that of partially amputating the semi-infinite cylinders associated with the $z_{1}$ and $z_{2}$ coordinates and gluing their boundaries, leaving a finite intermediate cylinder of length $-\frac{\ell_{s}}{2 \pi} \ln |t|$ and circumference equal to the systole $\ell_{s}$. The new surface can be shown to inherit a minimal-area metric through this sewing operation, guaranteeing consistency. The basic intuition proves correct: the new metric satisfies all length conditions ${ }^{3}$ and its area cannot be lowered because it would imply that the constituent surfaces would admit metrics with lower area than that of their minimal-area metrics. The surface built in Figure 3 is a four-punctured sphere with a minimal area metric. It corresponds to a Feynman graph with an internal propagator; this diagram, as the length and twist of the intermediate cylinder are varied, covers some region of the moduli space. Part of the moduli space is covered by surfaces that represent "vertex" contributions and are shown in Figure 4. Each surface in the vertex is an elementary four-point interaction and is constructed by gluing four semi-infinite cylinders on a tetrahedron graph. The perimeter of each of the faces of the tetrahedron must equal the systole length: $a+b+c=\ell_{s}$. By varying the length parameters $a, b$, and $c$, while guaranteeing that no curve becomes too short, one covers the vertex region $\mathcal{V}_{\mathbf{0 , 4}}$ of the moduli space $\mathcal{M}_{\mathbf{0 , 4}}$. Together, the two types of Feynman diagrams construct all surfaces in the moduli space of four punctured spheres.

For further illustration consider the moduli space $\mathcal{M}_{1,1}$ of once-punctured tori, relevant to oneloop tadpoles mentioned in Figure 2. This tadpole amplitude computes one-point functions of vertex operators and is composed of a Feynman diagram and a vertex $\mathcal{V}_{1,1}$. The Feynman diagram uses the three-string vertex - that is, the three-punctured sphere with the minimal area metric-and glues two of the punctures together via the identification $z_{1} z_{2}=t$ of the local coordinates with $|t|<1$, which as explained above introduces a cylinder of length $-\frac{\ell_{s}}{2 \pi} \ln |t|$ and circumference equal to the systole $\ell_{s}$ (see Figure 5). For small enough $|t|$ this operation produces metrics of minimal area with systole $\ell_{s}$. It is clear, however, that as $|t| \rightarrow 1$ gluing will fail to produce surfaces with an allowed minimal area metric: as the length of the cylinder goes to zero, noncontractible closed curves shorter than $\ell_{s}$ will appear. It is simple to figure out the subset of $|t|<1$ that generates minimal area metrics, but quite a bit harder [28] to determine the part of the moduli space $\mathcal{M}_{1,1}$ that these surfaces cover. They certainly fail to cover it all: not all minimal area metrics on $\mathcal{M}_{1,1}$ arise from a Jenkins-Strebel (JS) quadratic differential. The stubs that must be included in the three-string vertex (see footnote 3) imply that effectively the Feynman region $\mathcal{F}_{1,1}$ is generated by a cutoff region $|t|<e^{-2 \pi}$. All the surfaces in $\mathcal{F}_{1,1}$ have minimal area metrics arising from a JS quadratic differential. But, unavoidably, not all of the fundamental domain is covered. The missing region corresponds to the string vertex $\mathcal{V}_{1,1}$, shown with darker shade in Figure 5(c). The minimal area metrics on these conformal structures are not known, but they can be constructed with the methods of this paper. In particular, in the companion paper [22] we construct the minimal area metric on the simplest surface in $\mathcal{V}_{1,1}$, the square torus $\tau=i$.

\footnotetext{
${ }^{3}$ In order to construct higher genus diagrams without violating the length conditions one must redefine all the vertices by introducing stubs. This effectively means that the local coordinates are defined using the geodesic on the cylinder a distance $\ell_{s} / 2$ away from the last geodesic. Thus any gluing operation will automatically introduce a cylinder $\ell_{s}$ long, preventing the appearance of new short geodesics. A Riemann surface whose minimal-area metric does not contain an internal cylinder of length at least $\ell_{s}$ is considered a vertex, while one that does contain such a cylinder is built as a Feynman diagram out of vertices and propagators. For more details see [3].
} 

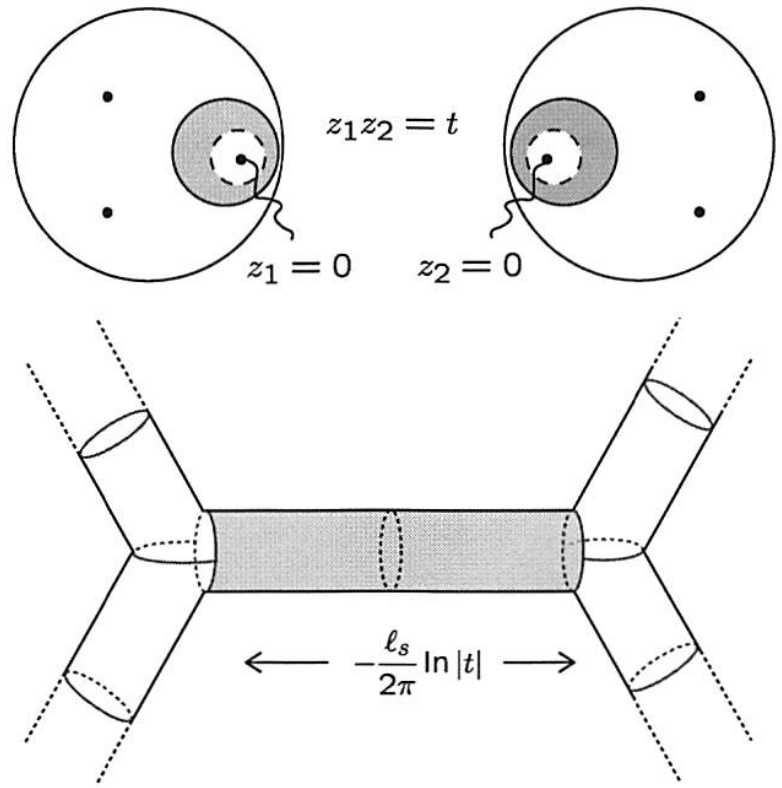

Figure 3: Two three-punctured spheres are glued by the identification $z_{1} z_{2}=t$ where $|t| \leq 1$, and $z_{1}$ and $z_{2}$ are, respectively, coordinates around a marked point on the left and on the right sphere. If the spheres are equipped with minimal area metrics each one looks like three semi-infinite cylinders coming together. In this case the cylinders with $z_{1}$ and $z_{2}$ coordinates are partially amputated and glued so that a cylinder of length $-\frac{\ell_{s}}{2 \pi} \ln |t|$ remains (shown shaded). The resulting four-punctured sphere is equipped with a minimal area metric.
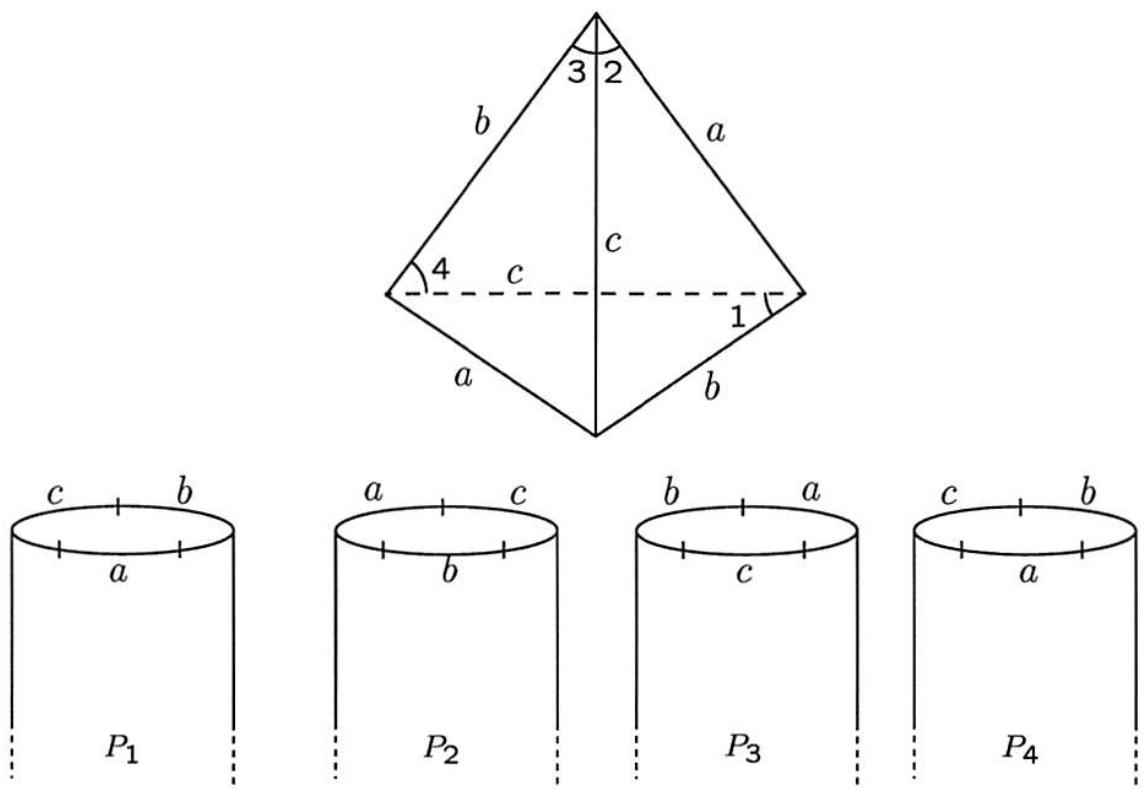

Figure 4: For some four-punctured spheres the minimal area metric arises by gluing the edges of four semiinfinite cylinders on the faces of a tetrahedron graph. Here $a, b, c$ are length parameters on the graph and $a+b+c=\ell_{s}$. In order not to have closed curves that violate the length conditions one must also have $a+b, b+c$, and $c+a$ all greater than or equal to $\ell_{s} / 2$. 


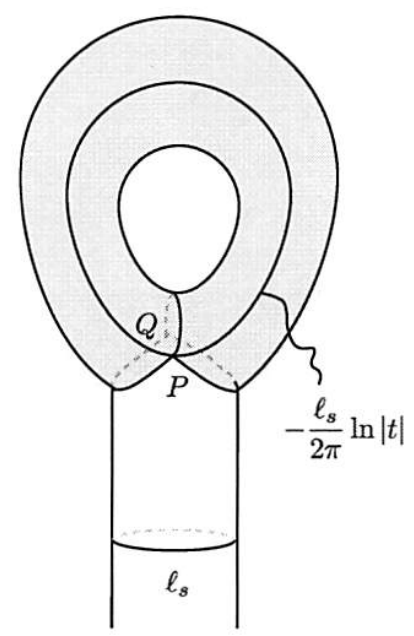

(a)

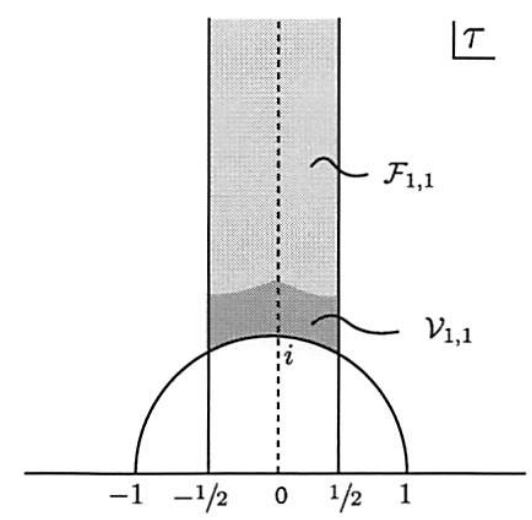

(c)

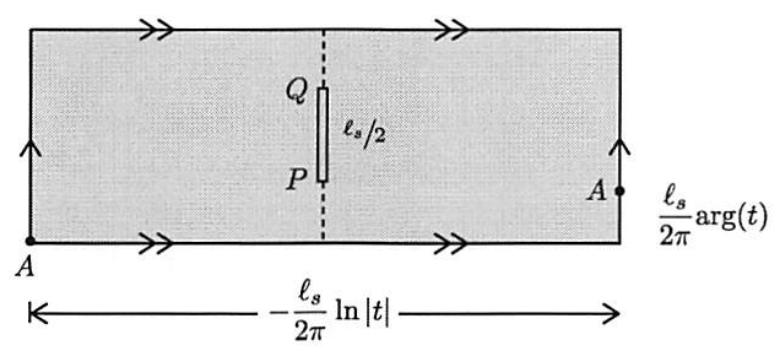

(b)

Figure 5: (a) The Feynman graph obtained by gluing a cylinder on the three-string vertex through the identification $z_{1} z_{2}=t$, with $|t|<e^{-2 \pi}$. This effectively introduces a cylinder of systolic circumference and length $-\frac{\ell_{s}}{2 \pi} \ln |t|$ greater than $\ell_{s}$. (b) The cylinder region shown in detail, including the effect of the twist angle $\arg (t)$. The semi-infinite cylinder representing the puncture is attached at the slit $P Q$. (c) The moduli space $\mathcal{M}_{1,1}$ of once-punctured tori (shaded) and a qualitative sketch of the Feynman region $\mathcal{F}_{1,1}$ and the (darker) vertex region $\mathcal{V}_{1,1}$.

The minimal-area metric is known for all genus-zero surfaces with $n \geq 3$ marked points [3]. In this case the metric is locally flat, has negative curvature conical singularities at some special points, and arises from a Jenkins-Strebel quadratic differential. Let regular points be the points on the surface that are not conical singularities nor marked points. The key property of these metrics is that going through every regular point there is a unique systolic geodesic. These are the socalled horizontal trajectories of the quadratic differential. At any conical singularity more than one saturating geodesic goes through. This knowledge of the genus-zero minimal-area metrics suffices to construct the classical closed string field theory and determines all the string vertices of the classical theory.

Equipped with the classical string vertices and the propagator, the Feynman rules allow us to build loop diagrams. These diagrams will build some but not all of the Riemann surfaces in the moduli spaces $\mathcal{M}_{g, n}$, with $g \geq 1$. All the built surfaces will share the properties of the genus-zero metrics: the metrics are flat except for conical singularities and there is a single systolic geodesic through each regular point. The missing surfaces, in general, will have a different kind of minimal- 
area metric: we expect them to have regions where through every point there is more than one systolic geodesic. These metrics are unknown and have not yet been proven to exist. The purpose of this paper is to develop methods to find these metrics and learn about their properties. We will not attempt here a proof of existence, but such a proof would be important progress as it would establish the existence of a closed string field theory! Indeed, even if we did not know the metrics, if they exist and satisfy some weak conditions, they would fulfill the three requirements above and thus ensure that a string field theory exists.

It is prudent to note that there are non-canonical proposals that possibly yield string field theories. One of them, based on symmetric, factorizable quadratic differentials was formulated by Sonoda and Zwiebach [29]. The construction is not without some (weak) mathematical assumptions and may require an adjustment of the propagator at each order of perturbation theory. Another option, recently investigated by Moosavian and Pius [30], is to use hyperbolic metrics. There is an obvious challenge here: hyperbolic metrics are not consistent with sewing. Nevertheless, it seems possible, by non-canonical deformations and a recursive procedure, to adjust the local coordinates to obtain consistency with sewing. In this paper we will not discuss these attempts.

Suppose we knew the minimal-area metrics exist, but their explicit form for each surface is complicated. It seems quite likely that the minimal-area metrics display some regularities or patterns regarding systolic geodesics. Let $U_{n}$ denote the subset of points in a Riemann surface where exactly $n$ systolic geodesics go through. We expect that each minimal-area surface will have regions $U_{1}, U_{2}, \ldots, U_{k}$ where $k$ depends on the genus and the number of marked points. Systole-length geodesics in the same homotopy class form bands that foliate the surface. The parameters associated with these bands include a suitably defined height, and perhaps angles formed by different intersecting bands. Such parameters could help construct a new decomposition of the moduli spaces of Riemann surfaces. It is also plausible that a number of questions in string field theory would only require partial information about the minimal-area metrics.

Of course, it would be ideal to know the explicit form of all minimal-area metrics. In principle, however, for any fixed surface the exact form of the metric is not needed on much of the surface. Arbitrary off-shell computations and the definition of the string field theory action only require the explicit form of the local coordinates at the marked points, and that requires finding the last systolic geodesic homotopic to each of the marked points on the surface. The value of the metric outside the coordinate disks does not affect anything.

\subsection{Mathematical context}

Minimal-area problems have a long history in the theory of Riemann surfaces. They led to the definition of the extremal length conformal invariant $\lambda$ associated to a collection of curves $\Gamma$ on a surface $M$ (see [9]). To compute $\lambda$ one minimizes the area of $M$ over the conformal metric while keeping all curves in $\Gamma$ longer than a constant $\ell_{s}$ identified as the systole. The conformal invariant $\lambda(\Gamma)$ is then given by the ratio of the squared systole and the minimal area. A useful test by Beurling [9] can be used to tell if a candidate metric is of minimal area, and a partial converse was given in [26]. The specification of $\Gamma$ is usually done by including some homotopy classes of curves on it.

The minimal-area metric can be shown to exist and arises from Jenkins-Strebel quadratic differentials whenever the chosen homotopy classes of curves in $\Gamma$ have representatives that do not 
intersect [4]. The extremal metric is known even when different length conditions are placed on the various homotopy classes in $\Gamma$. The homotopy classes can include those homotopic to the marked points and the required regularization of the area is inspired by Teichmüller's treatment of the modulus of a punctured disk [4]. The minimal-area problem for closed string field theory is much harder because $\Gamma$ includes all homotopy classes of curves, and therefore there are infinite instances of pairs of homotopy classes where representatives always intersect. As soon as such pairs exist, the minimal-area metric can easily fail to arise from a quadratic differential. In the minimal-area metric there are systole-length curves for some subset of the homotopy classes of curves. If these "realized" homotopy classes have representatives that do not intersect, then the minimal-area metric will arise from a quadratic differential. If realized homotopy classes intersect, the metric will almost always fail to arise from a quadratic differential. For genus zero, the minimal-area metric arises from a quadratic differential because realized homotopy classes cannot intersect. If two systole-length curves in different homotopy classes intersect at one point, they would have to intersect in a second point because each geodesic cuts a genus zero surface into two pieces. But one can prove that two non-homotopic systoles cannot generally intersect at more than one point [3].

Another perspective on the minimal-area problem arises from systolic geometry [10]. Gromov introduced the notion of essential $n$-dimensional manifolds $M$ as those for which the systolic volume

$$
\frac{\operatorname{Vol}(M, g)}{(\operatorname{sys}(M, g))^{n}}
$$

has a lower bound on the space of Riemannian metrics $g$ on $M$ and the bound only depends on the topology of $M$ [13]. When the manifold is two dimensional, the above ratio coincides with the quantity that one considers for the calculation of the extremal length. In the extremal length problem, the conformal structure of the surface is fixed and the metrics are restricted to be conformal. In minimizing the above ratio, the conformal structure is not kept fixed as one works with all Riemannian metrics and there is just one minimum ratio for each genus. Of course, in systolic geometry one can study "conformal systolic inequalities" [14] and this is exactly the same subject considered in the theory of Riemann surfaces. The highest lower bound for the systolic ratio is known for genus one, and it is attained for the torus $\tau=e^{i \pi / 3}$ equipped with a flat metric [31]. The highest lower bound for the systolic ratio is not known for genus two, or in fact for any $g \geq 2$. For surfaces, and allowing the complex structure to vary, Calabi [15] showed that there is no region $U_{1}$ with just one band of systole-length geodesics and, in $U_{2}$ the two bands of systolic geodesics are perpendicular everywhere and the metric is flat. He described the foliations using calibrations, as elaborated by Bryant [17]. Calabi's results, however, do not apply for the conformal case. There are many examples with regions $U_{1}$, and the proof of flatness for $U_{2}$ does not extend to the conformal case.

\section{Review of convex programming and max flow-min cut}

In this section we review basic facts about convex programs and their duals that will be used in the rest of the paper. We will state the relevant results mostly avoiding proofs. For a somewhat more detailed review of similar facts, the reader should consult [20]. For more details as well as proofs, the book by S. Boyd and L. Vandenberghe [18] is highly recommended. We also discuss the max flow-min cut theorem as applied to calibrations and curves in homology, both as an illustration of these ideas and because it is the starting point for our investigations. 


\subsection{Convex programs}

In discussing convex programs we need the terminology of convex and affine sets, and convex and affine functions. Consider a set $C \subseteq \mathbb{R}^{n}$. We will denote a point in $\mathbb{R}^{n}$ as $y$. The set $C$ is affine if the full line going through any two distinct points in $C$ lies in $C$. A set $C$ is convex if the line segment joining any two distinct points in $C$ lies in $C$. A function $f: C \rightarrow \mathbb{R}$ is convex if its domain $C$ is a convex set and for all $y_{0}, y_{1}$ in that domain

$$
f\left(t y_{0}+(1-t) y_{1}\right) \leq t f\left(y_{0}\right)+(1-t) f\left(y_{1}\right) \quad \forall t \in[0,1] .
$$

A function $f$ is said to be concave if $-f$ is convex. A function $h: \mathbb{R}^{n} \rightarrow \mathbb{R}$ is affine if it is the sum of a linear function and a constant function. Affine functions satisfy equation (3.1) with the inequality replaced by equality. Thus affine functions are convex and also concave. A function is strictly convex if the strict inequality holds for $t \in(0,1)$. It is natural to search for the minimum of a convex function and for the maximum of a concave function.

Some operations preserve convexity. The non-negative weighted sum of a set of convex functions is itself a convex function. If $f_{1}$ and $f_{2}$ are convex functions, their pointwise maximum $f$, defined for each $y$ by $f(y)=\max \left\{f_{1}(y), f_{2}(y)\right\}$, is a convex function whose domain is the intersection of the domains of $f_{1}$ and $f_{2}$. Similarly, the pointwise minimum of two concave functions is concave. These statements obviously generalize to the pointwise maximum (minimum) of any number of convex (concave) functions. A special case we will use below involves a continuous family of affine functions of $y$ indexed by a variable $w \in \mathcal{B}$ : If $f(w, y)$ is affine in $y$ for all $w \in \mathcal{B}$, then

$$
g(y):=\inf _{w \in \mathcal{B}} f(w, y)
$$

is a concave function of $y$ on the domain where the infimum is finite.

Now consider the following optimization problem presented in standard form:

$$
\begin{array}{cl}
\text { Minimize } & f_{0}(y) \quad \text { over } y \in \mathcal{D} \\
\text { subject to } & f_{i}(y) \leq 0 \quad i=1, \ldots, m \\
& h_{j}(y)=0 \quad j=1, \ldots, p
\end{array}
$$

In this program $y \in \mathbb{R}^{n}$ is the variable we minimize over. Here $f_{0}(y)$ is the objective function, the $f_{i}$ 's with $i \geq 1$ define the inequality constraints, and the $h_{j}$ 's define the equality constraints. The domain $\mathcal{D}$ of the problem is a subset of $\mathbb{R}^{n}$ defined as the common domain of the functions $f_{i}(i=0, \ldots, m)$ and $h_{j}(j=1, \ldots, p)$. The feasible set $\mathcal{F} \subseteq \mathcal{D}$ is defined to be the subset of the domain in which the constraints hold:

$$
\mathcal{F}=\left\{y \mid y \in \mathcal{D}, f_{i}(y) \leq 0 \forall i, h_{j}(y)=0 \forall j\right\}
$$

The optimal value $p^{*}$ is the minimum value of the objective over the feasible set $\mathcal{F}$ :

$$
p^{*}=\inf _{y \in \mathcal{F}} f_{0}(y) .
$$

A point $y^{*} \in \mathcal{F}$ is said to be an optimal point if $f_{0}\left(y^{*}\right)=p^{*}$. Since, in much of this paper, we will be working in function spaces rather than in $\mathbb{R}^{n}$, we will also use the terms optimal configuration and solution, to avoid confusion with the points of the underlying manifold. (In the optimization 
literature, "solution" generally refers to the optimal value $p^{*}$; however, it seems more natural in the physics context to use the word to refer to the optimal point or configuration.) Note that, in general, an optimal point need not exist, and if one exists it need not be unique.

The above problem is a convex program if the objective $f_{0}$ and inequality constraint functions $f_{i}$ are convex functions over a convex domain $\mathcal{D}$, and the $h_{i}$ are affine functions. It follows that the feasible set $\mathcal{F}$ is also convex. For a convex program, any local minimum is in fact a global minimum. If $f_{0}$ is strictly convex, then the optimal point $y^{*}$ (if it exists) is unique, otherwise it need not be. However, the set of optimal points $\left\{y^{*} \in \mathcal{F} \mid f\left(y^{*}\right)=p^{*}\right\}$ is necessarily convex.

We will also refer to the maximization problem

$$
\begin{array}{cl}
\text { Maximize } & g_{0}(y) \quad \text { over } y \in \mathcal{D} \\
\text { subject to } & g_{i}(y) \geq 0 \quad i=1, \ldots, m, \\
& h_{j}(y)=0 \quad j=1, \ldots, p,
\end{array}
$$

where the functions $g_{i}(i=0, \ldots, m)$ are concave, as a convex program, since it is obviously related to the convex program (3.3) just by taking $f_{i}(y)=-g_{i}(y)(i=0, \ldots, m)$.

Symmetries of a convex program can greatly aid their solution. Suppose that a convex program is invariant under a finite or compact group $G$ of affine transformations of $\mathbb{R}^{n}$; that is, the domain $\mathcal{D}$, the objective $f_{0}$, the set of inequality constraint functions $\left\{f_{i}\right\}$, and the set of equality constraint functions $\left\{h_{j}\right\}$ are all invariant under the action of $G$. Then, acting on any feasible point $y$ by any element $g$ of $G$ produces a point $g(y)$ that is also feasible and has the same value of the objective $f_{0}$. Furthermore, by the convexity of the feasible set, the average $\langle y\rangle_{G}$ over the $G$ action is also feasible, and by the convexity of $f_{0}$, the value of $f_{0}$ for the average is no larger than for $y$ :

$$
f_{0}\left(\langle y\rangle_{G}\right) \leq f_{0}(y) .
$$

This average $\langle y\rangle_{G}$ is clearly a fixed point of $G$. Therefore, when minimizing we can impose the symmetry at the outset without changing the value of $p^{*}$. In other words, we can reduce the domain $\mathcal{D}$ to the locus of $G$-fixed points within $\mathcal{D}$. Since $G$ acts affinely, this locus is a convex set. Such a reduction by a symmetry can make it much easier to solve the problem, as we will see at several points in this paper and the sequel [22].

\subsection{Lagrangian duality}

Lagrangian duality is a method for transforming a constrained optimization problem into another one, written in terms of a different set of variables, that gives information about the solution to the first one. In fact, under fairly general circumstances, the two problems are equivalent, in the sense that they have the same optimal value. The method involves introducing Lagrange multipliers for the constraints and then solving for the original variables, leaving a program expressed in terms of the Lagrange multipliers. In the process, a program involving minimizing a convex objective, such as (3.3), becomes a program involving maximizing a concave objective, and vice versa. In this context, the original program is referred to as the primal and the new one as the dual. We will now describe the method in more detail, taking (3.3) as our primal. 
The Lagrangian function $L(x, \lambda, \nu)$ is built by adding to the objective of the primal a sum of terms with Lagrange multipliers $\lambda_{i}$ and $\nu_{j}$ multiplying the constraint functions:

$$
L(y, \lambda, \nu):=f_{0}(y)+\sum_{i=1}^{m} \lambda_{i} f_{i}(y)+\sum_{j=1}^{p} \nu_{j} h_{j}(y) .
$$

Since $L(y, \lambda, \nu)$ is, for any fixed $y$, an affine function of $\lambda$ and $\nu$, minimizing over $y$ yields a concave function of $\lambda$ and $\nu$ called the Lagrange dual function or dual objective $g_{0}(\lambda, \nu)$ :

$$
g_{0}(\lambda, \nu):=\inf _{y \in \mathcal{D}} L(y, \lambda, \nu)
$$

Its domain $\mathcal{D}^{\prime}$ is the set of points $(\lambda, \nu)$ on which the infimum is finite. One can prove that for any $\nu$ and any $\lambda \geq 0$ (i.e. $\lambda_{i} \geq 0$ for all $i$ ) the dual objective provides a lower bound for the optimal value $p^{*}$ of the primal:

$$
g_{0}(\lambda \geq 0, \nu) \leq p^{*}
$$

Since $g_{0}(\lambda, \nu)$ is a concave function of $\lambda$ and $\nu$, it makes sense to look for its maximum. Indeed, one defines the dual program:

$$
\begin{array}{cl}
\text { Maximize } & g_{0}(\lambda, \nu) \quad \text { over }(\lambda, \nu) \in \mathcal{D}^{\prime}, \\
\text { subject to } & \lambda_{i} \geq 0, \quad i=1, \ldots, m .
\end{array}
$$

This is a convex optimization problem of the form (3.6) (regardless of whether the primal is convex). The dual feasible set is the set

$$
\mathcal{F}^{\prime}:=\left\{(\lambda, \nu) \in \mathcal{D}^{\prime} \mid \lambda \geq 0\right\}
$$

We have a dual optimal value $d^{*}$ and dual optimal point (or configuration) $\left(\lambda^{*}, \nu^{*}\right) \in \mathcal{F}^{\prime}$ if

$$
d^{*}:=\sup _{(\lambda, \nu) \in \mathcal{F}^{\prime}} g_{0}(\lambda, \nu)=g_{0}\left(\lambda^{*}, \nu^{*}\right)
$$

It follows from (3.10) that

$$
d^{*} \leq p^{*}
$$

this relation is called weak duality. We have strong duality if the primal and dual optimum values are the same: $d^{*}=p^{*}$. Strong duality holds in a variety of situations but can be guaranteed when the primal is convex and the Slater condition is satisfied: there exists a feasible point $x$ in the interior of $\mathcal{D}$ where the inequality constraints are strictly satisfied $\left(f_{i}(y)<0\right.$ for $i=1, \ldots, m, h_{j}(y)=0$ for $j=1, \ldots, p)$.

Suppose strong duality holds, and let $y^{*}$ and $\left(\lambda^{*}, \nu^{*}\right)$ be primal and dual optimal points respectively. The inequality constraints and their Lagrange multipliers then satisfy complementary slackness:

$$
\lambda_{i}^{*} f_{i}\left(y^{*}\right)=0 \text { for each } i .
$$

Note that the primal and dual optimal points may not be unique. However, since (3.15) holds for any primal and dual optimal points, we have the following:

1. If there exists a primal optimal point $y^{*}$ such that $f_{i}\left(y^{*}\right)<0$, then for all dual optimal points $\lambda_{i}^{*}=0$ (slack in the constraint implies no slack in the multiplier). 
2. If there exists a dual optimal point $\left(\lambda^{*}, \nu^{*}\right)$ such that $\lambda_{i}^{*}>0$, then for all primal optimal points $f_{i}\left(y^{*}\right)=0$ (slack in the multiplier implies no slack in the constraint).

In the case $f_{i}\left(y^{*}\right)=0$, one says that the constraint is active (or saturated). One can also show that, for any dual optimal $\left(\lambda^{*}, \nu^{*}\right)$, any primal optimal $y^{*}$ minimizes $L\left(y, \lambda^{*}, \nu^{*}\right)$ :

$$
\inf _{y \in \mathcal{D}} L\left(y, \lambda^{*}, \nu^{*}\right)=L\left(y^{*}, \lambda^{*}, \nu^{*}\right) .
$$

This follows from strong duality as follows:

$$
\inf _{y \in \mathcal{D}} L\left(y, \lambda^{*}, \nu^{*}\right)=g_{0}\left(\lambda^{*}, \nu^{*}\right)=d^{*}=p^{*}=f_{0}\left(y^{*}\right)=L\left(y^{*}, \lambda^{*}, \nu^{*}\right) .
$$

In the rest of this paper we will be working mostly not in $\mathbb{R}^{n}$ but in infinite-dimensional function spaces. In doing so, we will simply assume that the above results carry over, without worrying too much about the functional analysis that would be necessary to prove our claims rigorously.

\subsection{Calibrations and the max flow-min cut theorem}

In network theory one often considers a source node $s$ and a sink node $t$ connected by a graph with some number of nodes and edges. Each edge is assigned a capacity, the maximum flux it can handle. With this graph one can pose two different problems that are nontrivially related. The first is a max flow problem. A flow is an assignment of fluxes to each edge of the graph consistent with their capacity. The assignment must respect conservation (flux in $=$ flux out) at each node except $s$ and $t$. In the max flow problem one searches for the flow with maximum flux from the source $s$ to the sink $t$. The second problem is a min cut problem. A cut is a partition of the nodes into two sets $S$ and $T$, with $S$ containing $s$ and $T$ containing $t$. The capacity of the cut is the sum of capacities of the edges connecting $S$ and $T$. The min cut problem asks for the cut of minimum capacity. The max flow-min cut (MFMC) theorem states that for any network the flux of the max flow equals the capacity of the min cut. These two problems can be stated as linear programs related by strong duality.

In this paper we will make extensive use of a closely analogous theorem in the setting of Riemannian manifolds. In this theorem, the role of a flow is played by a norm-bounded divergenceless vector field and the role of a cut is played by a hypersurface in a specified homology class. The proof of the theorem, which hinges on strong duality of convex programs, can be found in the references [19]. Here we will explain the setup, state the theorem, and sketch its proof. Our sketch focuses on the aspects of the proof involving convex optimization and skips over those involving geometric measure theory (properly defining minimal surfaces, proving their existence, etc.). Several of the techniques and intermediate results in the proof will be used in the rest of the paper. In reference [20] one can find a detailed discussion of the MFMC theorem focused on the case when the homology class is defined by a region of the manifold's boundary, the situation relevant to the study of holographic entanglement entropy.

\subsubsection{Setup and statement}

Let $M$ be a connected Riemannian manifold, possibly with boundary. In what follows $M$ and all submanifolds are assumed compact and oriented. While in most of this paper $M$ is two-dimensional, 
in this subsection $M$ may have arbitrary dimension $d$. We define a flow as a vector field $v$ obeying

$$
\nabla_{\mu} v^{\mu}=0, \quad|v| \leq 1
$$

everywhere. Let $C$ be an integral $d-1$ homology class, $C \in H_{d-1}(M, \mathbb{Z}) \cdot{ }^{4}$ A cut is a hypersurface $m$ in the class $C$. The volume form $\omega=\sqrt{g} d^{d} x$ on $M$ can be pulled back onto $m$ to give its area form $\omega_{\|}$, whose integral is the total area of $m:{ }^{5}$

$$
\operatorname{area}(m):=\int_{m} \omega_{\|} .
$$

We now consider the flux of a flow $v$ through a cut $m$,

$$
\operatorname{flux}_{m}(v):=\int_{m} \omega_{\|} n_{\mu} v^{\mu},
$$

where $n_{\mu}$ is the unit normal to $m$. By virtue of the divergenceless condition, this flux is independent of $m$, so we can write instead flux $x_{C}(v)$. On the other hand, the norm bound $|v| \leq 1$ implies $n_{\mu} v^{\mu} \leq 1$; integrating this inequality over $m$ shows that the flux is bounded above by the area of $m$. All in all, for any flow $v$ and any cut $m$ we have

$$
\operatorname{flux}_{C}(v) \leq \operatorname{area}(m)
$$

The MFMC theorem asserts that this inequality is tight:

$$
\sup _{v} \operatorname{flux}_{C}(v)=\inf _{m \in C} \operatorname{area}(m) \text {. }
$$

A flow $v^{*}$ that achieves the supremum is called a max flow and a cut $m^{*}$ that achieves the infimum is called a min cut. Under suitable regularity conditions, $v^{*}$ and $m^{*}$ exist; however, they are not necessarily unique.

An equivalent, and in some cases more convenient, language for talking about flows is that of calibrations [36]. A $p$-calibration is a $p$-form $u$ that is closed, $d u=0$, and has norm $|u| \leq 1$. The norm bound implies that for any $p$-dimensional submanifold $m$ the period of $u$ along $m$ is bounded by the area of $m$ :

$$
\int_{m} u \leq \int_{m} \omega_{\|} \cdot
$$

If $u=\omega_{\|}$everywhere on $m$ we say that $u$ calibrates $m$. Clearly, if $u$ calibrates $m$ then the bound (3.23) is saturated. Conversely, if $u$ is a calibration and (3.23) is saturated, then $u$ calibrates $m$.

The relation

$$
u=*\left(g_{\mu \nu} v^{\mu} d x^{\nu}\right),
$$

with $*$ the Hodge star, gives a one-to-one map between flows $v$ and $(d-1)$-calibrations $u ; \nabla_{\mu} v^{\mu}=0$ is equivalent to $d u=0$, and $|v| \leq 1$ is equivalent to $|u| \leq 1$. While the condition $\nabla_{\mu} v^{\mu}=0$ involves the metric via the Christoffel symbol, the condition $d u=0$ does not. In the rest of the paper we will be varying the metric. It will therefore be more convenient for us to use the language of calibrations,

\footnotetext{
${ }^{4}$ With minor modifications, everything in this subsection can be generalized to the case where $C$ is a homology class relative to some subset of $M$.

${ }^{5}$ In the case $d=2$ relevant to the rest of the paper, a "hypersurface" is a curve, and its "area" in the sense of (3.19) is its length.
} 
where the metric only appears in the constraint $|u| \leq 1$. When the metric is fixed, however, the two notions are interchangeable.

Under the relation (3.24), we have

$$
\operatorname{flux}_{C}(v)=\int_{C} u
$$

where by the closedness of $u$ we needn't specify on which representative of $C$ we evaluate the period of $u$. Therefore, in the language of calibrations, the MFMC theorem reads

$$
\sup _{u} \int_{C} u=\inf _{m \in C} \operatorname{area}(m) .
$$

A calibration $u^{*}$ that achieves the supremum is called a max calibration. Like the max flow $v^{*}$, it is not necessarily unique. However, any max calibration must calibrate every min cut $m^{*}$, and therefore is fully determined on the locus of min cuts. Elsewhere, however, it is underdetermined. In particular, for any point $x$ not on any min cut, there exists a max calibration $u^{*}$ such that $\left|u^{*}(x)\right|<1$. We will take this fact as intuitively clear (although the reader can find an argument in subsection 3.4 of [20]). This will become important below when we use max calibrations to find min cuts.

\subsubsection{Proof}

We will now sketch the proof of (3.26). Here is the general outline. First, we note that the conditions defining a calibration, $d u=0,|u| \leq 1$, are convex, and the functional $\int_{C} u$ is linear in $u$. Therefore we can write a convex program, which we call the max flow program, whose optimal value is the lefthand side of (3.26). On the other hand, for the right-hand side, which we call the min cut program, the objects being minimized over-hypersurfaces in the homology class $C$-do not naturally form a convex set. We therefore rewrite the problem as a minimization over representatives of the Poincaré dual cohomology class, which are closed one-forms. These do form a convex set, on which we can define a convex objective that is a generalized "area" functional. We will call this the convex min cut program. A non-trivial step is to show that it is equivalent to the original min cut problem. Furthermore, it is related by strong duality to the max flow program. We will thus show

$$
\max \text { flow }=\text { convex } \min \text { cut }=\min \text { cut } .
$$

Here $=$ means the programs are equivalent, i.e. have the same optimal value. We will go from left to right in this equation: write down the max flow program, dualize it in order to get the convex min cut program, and finally show that the latter reduces to the original min cut program.

We first consider the case where $M$ is closed, in order to eliminate certain complications that would distract from the main story. At the end we will fill in the generalization to manifolds with boundary.

From max flow to convex min cut: To write the left-hand side of (3.26) in terms of a convex program, we let the domain be the set of $(d-1)$-forms on $M$, which is clearly a convex set, and we impose the definition of a calibration, $d u=0$ and $|u| \leq 1$, as explicit constraints. The first is a linear equality constraint, while the second is a convex inequality constraint. For the objective, we would like to write $\int_{C} u$. However, this is only well-defined if $u$ is closed-otherwise the integral depends 
on the choice of representative of $C$-and we need an objective that is well-defined on the entire domain, i.e. before imposing the constraints. We could resolve this problem by picking an arbitrary representative of $C$. However, it will turn out to be more convenient to appeal to Poincaré duality and instead choose a representative $\eta_{0}$ of the dual cohomology class $\tilde{C} \in H^{1}(M)$. This means that, for any closed $(d-1)$-form $u$,

$$
\int_{C} u=\int_{M} u \wedge \eta_{0}
$$

The right-hand side provides an objective that is well-defined for any $(d-1)$-form and equals the one we want for closed ones. The max flow program then reads

$$
\begin{aligned}
\text { Max flow: Maximize } \quad \int_{M} u & \wedge \eta_{0} \quad \text { over } u((d-1) \text {-form }) \\
\text { subject to } \quad 1-|u| & \geq 0, \\
d u & =0, \quad \forall x \in M .
\end{aligned}
$$

We will follow the convention of indicating the type of each variable in a program (constant, function, form, etc.) in parentheses after its name. The position $x$ in the manifold is playing the role of the indices $i$ and $j$ in the equality and inequality constraints; henceforth we will not write $\forall x \in M$ in each program.

We now dualize the max flow program. Since $d u$ is a top-form, it has only one independent component. As a result, a single Lagrange multiplier $\psi$, a scalar function on $M$, suffices for the constraint $d u=0$. We introduce a second scalar Lagrange multiplier $\phi \geq 0$ for the inequality constraint $1-|u| \geq 0$. The Lagrangian functional is

$$
L[u, \psi, \phi]=\int_{M} u \wedge \eta_{0}+\int_{M}\left((-1)^{d} \psi d u+\omega \phi(1-|u|)\right),
$$

where again $\omega$ is the volume form and the factor $(-1)^{d}$ is included to simplify future equations. We now wish to maximize $L$ with respect to $u$, where $u$ is now an arbitrary $(d-1)$-form. We can do the maximization pointwise on $M$ if we first integrate the $\psi d u$ term by parts to get the derivative off of $u$ :

$$
L[u, \psi, \phi]=\int_{M}\left(u \wedge\left(\eta_{0}+d \psi\right)-\omega \phi|u|+\omega \phi\right)=\int_{M} \omega\left(I_{u}+\phi\right),
$$

where

$$
I_{u}=(-1)^{d-1} v^{\mu} \eta_{\mu}-\phi|v|, \quad \eta:=\eta_{0}+d \psi,
$$

and the vector $v$ is defined in terms of $u$ by (3.24). Maximizing $L$ with respect to $u$ is equivalent to maximizing $I_{u}$ with respect to $v$ at each point. One can convince oneself that $I_{u}$ is bounded above if and only if $\phi \geq|\eta|$, and in this case its maximum is zero. This inequality on $\phi$ allows us to drop the earlier constraint $\phi \geq 0$. So the dual program is

$$
\begin{array}{ll}
\text { Minimize } & \int_{M} \omega \phi \text { over } \psi, \phi \text { (functions), } \\
\text { subject to } & \phi \geq\left|\eta_{0}+d \psi\right|
\end{array}
$$

Clearly the minimum with respect to $\phi$ is achieved by setting $\phi=\left|\eta_{0}+d \psi\right|$. This leaves the program

$$
\text { Minimize } \quad \int_{M} \omega\left|\eta_{0}+d \psi\right| \text { over } \psi \text { (function) }
$$


As expected, the objective is convex in $\psi$. Moreover, there are no explicit constraints.

For any function $\psi$ the one-form $\eta_{0}+d \psi$ is in the class $\tilde{C}$, and conversely any one-form $\eta$ in that class can be written as $\eta_{0}+d \psi$ for some function $\psi$; in other words the map $\psi \mapsto \eta_{0}+d \psi$ from functions to $\tilde{C}$ is surjective. It is also affine. So we can change variables to $\eta$ and write (3.34) as

$$
\text { Convex min cut: Minimize } \int_{M} \omega|\eta| \text { over } \eta \in \tilde{C} \text {. }
$$

(The reason for the name will be explained shortly.) Strong duality holds because Slater's condition, stated below (3.14), holds: the $d-1$ form $u=0$ is feasible and strictly satisfies the inequality constraint $|u| \leq 1$. Therefore, the convex min cut program is equivalent to the max flow program. This establishes the first equality in (3.27).

From convex min cut to min cut: The min cut, by definition, is the solution to the following program:

$$
\text { Min cut: Minimize area }(m) \text { over } m \in C \text {. }
$$

Since the homology class $C$ is not a convex set, this is not a convex program. However, it is related to the convex min cut program (3.35) by convex relaxation. Convex relaxation essentially means turning an optimization problem with a non-convex feasible set into a convex program by embedding the feasible set into a larger convex set and extending the definition of the objective to a convex function on the new feasible set. In this case, we will use a map $\eta_{m}$ defined below to embed the non-convex set $C$ into the convex set $\tilde{C}$, and replace the area functional with the convex functional $\int_{M} \omega|\eta|$. In general, relaxation can result in a program with a lower optimal value than the original one. We will check, however, that this is not the case here: the convex min cut and original min cut programs have the same optimal values.

To relate the one-forms appearing in the convex min cut program to the hypersurfaces appearing in the min cut program, we recall that there is a map taking an arbitrary hypersurface $m$ to a oneform $\eta_{m}$ such that, for any (not necessarily closed) $(d-1)$-form $u$,

$$
\int_{m} u=\int_{M} u \wedge \eta_{m}
$$

In a local coordinate system $x^{\mu}(\mu=1, \ldots, d)$ in which $m$ is at $x^{d}=0$ and has orientation form $d x^{1} \wedge \cdots \wedge d x^{d-1}$, the one-form $\eta_{m}$ is given by ${ }^{6}$

$$
\eta_{m}=\delta\left(x^{d}\right) d x^{d}
$$

$\eta_{m}$ is sometimes called a "bump form" (see [33], section 6 for a more detailed discussion). It is clear that if $m$ is closed then $\eta_{m}$ is closed, and furthermore if $m \in C$ then $\eta_{m} \in \tilde{C}$. Working in the above local coordinate system, one can also show that $\omega\left|\eta_{m}\right|=\omega_{\|} \wedge \eta_{m}$, so

$$
\int_{M} \omega\left|\eta_{m}\right|=\operatorname{area}(m)
$$

\footnotetext{
${ }^{6}$ In (3.38), we assume that $m$ has unit multiplicity at $x^{d}=0$. More generally, if it has multiplicity $n_{+}$with orientation $d x^{1} \wedge \cdots \wedge d x^{d-1}$ and $n_{-}$with opposite orientation, then $\eta_{m}=\left(n_{+}-n_{-}\right) \delta\left(x^{d}\right) d x^{d}$.
} 
Taking the infimum over $m$ we get

$$
\inf _{m \in C} \operatorname{area}(m)=\inf _{m \in C} \int_{M} \omega\left|\eta_{m}\right| \geq \inf _{\eta \in \tilde{C}} \int_{M} \omega|\eta|
$$

We would have gotten an equality in (3.40) if $m \mapsto \eta_{m}$ were a surjective map to $\tilde{C}$. It is not, however: most one-forms in $\tilde{C}$ are not the bump form of any hypersurface in $C$. However, as we will now show, any $\eta \in \tilde{C}$ corresponds in some sense to a convex combination of hypersurfaces in $C$. By this we mean the following. Since $\eta$ is closed, by the Frobenius theorem it is hypersurfaceorthogonal. We will construct those hypersurfaces, which we will call $m_{\eta}(t)$, and show that they are in the class $C$, are parametrized by points on the circle $\mathbb{R} / \mathbb{Z}$, and have average area equal to $\int \omega|\eta|$. This will establish equality between the left- and right-hand sides of (3.40).

Let $\eta$ be a continuous one-form in $\tilde{C}$; we will treat one-forms that are discontinuous or have delta functions as limits of continuous ones. Since $\tilde{C}$ is the Poincaré dual of $C$, which is an integral homology class, the integral of $\eta$ over any closed curve $c$ equals the intersection number $\#(c, C)$, which is an integer. Therefore, given two points $x_{1,2} \in M$, the integral of $\eta$ along a path from $x_{1}$ to $x_{2}$ is independent of the choice of path up to an integer. Fixing a base point $x_{0}$, we define the continuous function $\Psi_{\eta}: M \rightarrow \mathbb{R} / \mathbb{Z}$ by

$$
\Psi_{\eta}(x)=\int_{x_{0}}^{x} \eta
$$

It is clear that $d \Psi_{\eta}=\eta$. (In fact, we could equivalently define $\Psi_{\eta}$ as the solution to $d \Psi_{\eta}=\eta$ with $\Psi_{\eta}\left(x_{0}\right)=0$.) Changing the base point $x_{0}$ just shifts $\Psi_{\eta}$ by an unimportant constant, so we will not indicate $x_{0}$ explicitly. Given a closed curve $c: \mathbb{R} / \mathbb{Z} \rightarrow M$, the map

$$
\Psi_{\eta} \circ c: \mathbb{R} / \mathbb{Z} \rightarrow \mathbb{R} / \mathbb{Z}
$$

has winding number

$$
\int_{c} d \Psi_{\eta}=\int_{c} \eta=\#(c, C)
$$

We will use this fact below.

Any connected subset of $M$ on which $\eta$ vanishes maps under $\Psi$ to a single point of $\mathbb{R} / \mathbb{Z}$. Therefore the locus $\{x \in M \mid \eta(x)=0\}$ maps to a set of isolated points in $\mathbb{R} / \mathbb{Z}$. Let $S \subseteq \mathbb{R} / \mathbb{Z}$ be the complement of this set. Since the isolated points form a set of measure zero, in integrals we will ignore the difference between $\mathbb{R} / \mathbb{Z}$ and $S$. For a point $t \in S$, define the level set $m_{\eta}(t)$ as the inverse image of $\Psi$ :

$$
m_{\eta}(t):=\Psi_{\eta}^{-1}(t) .
$$

This level set is a closed hypersurface on which $\eta \neq 0$; we fix its orientation by pulling the $(d-1)$ form $* \eta$ back to $m_{\eta}(t)$. The intersection number of any closed curve $c$ with $m_{\eta}(t)$ is the number of times (counted with signs) that $\Psi_{\eta} \circ c$ intersects $t$, which is the winding number of $\Psi_{\eta} \circ c$, which in turn as shown above is \# $(c, C)$. The intersection numbers of a hypersurface with a complete set of closed curves (representing a basis for $H_{1}(M, \mathbb{Z})$ ) fully determine its homology class; since these intersection numbers are the same for $m_{\eta}(t)$ as for $C, m_{\eta}(t)$ must be in the class $C$.

Finally, by the coarea formula,

$$
\int_{M} \omega|\eta|=\int_{M} \omega\left|d \Psi_{\eta}\right|=\int_{0}^{1} d t \operatorname{area}\left(m_{\eta}(t)\right)
$$


which implies

$$
\int_{M} \omega|\eta| \geq \inf _{m \in C} \operatorname{area}(m)
$$

Taking the infimum of (3.46) over $\eta$,

$$
\inf _{\eta \in \tilde{C}} \int_{M} \omega|\eta| \geq \inf _{m \in C} \operatorname{area}(m)
$$

Together with (3.40), which is the above inequality in the opposite direction, this establishes the equivalence of the min cut and convex min cut programs:

$$
\inf _{m \in C} \operatorname{area}(m)=\inf _{\eta \in \tilde{C}} \int_{M} \omega|\eta|
$$

Manifolds with boundary: The generalization of the proof of MFMC to manifolds with boundary requires only a minor modifications of the above, essentially just imposing certain boundary conditions.

On a manifold with boundary we must replace Poincaré duality with Poincaré-Lefschetz duality, which establishes an isomorphism between the homology group $H_{d-1}(M)$ and the cohomology group relative to the boundary $H^{1}(M, \partial M)$. Relative cohomology means that all forms are subject to the boundary condition that their pullback onto $\partial M$ vanishes. In particular, for us it means that every element $\eta$ of the dual class $\tilde{C}$ is normal to $\partial M$, and any two elements differ by an exact one-form $d \phi$ where $\phi$ vanishes on $\partial M$.

The max flow program (3.29) and the Lagrangian (3.30) are unchanged. However, when we integrate by parts, there is a new surface term $(-1)^{d} \int_{\partial M} \psi u$ which must be added to the right-hand side of (3.31). When we then maximize with respect to $u$ (which, recall, is unconstrained at this stage), this term is bounded above if and only if $\psi$ vanishes on $\partial M$, in which case the term vanishes. So the only change to the programs (3.33) and (3.34) is the addition of a boundary condition:

$$
\left.\psi\right|_{\partial M}=0 .
$$

This boundary condition is precisely what is required to ensure that $\eta:=\eta_{0}+d \psi$ is an element of $\tilde{C}$, and conversely that every element of $\tilde{C}$ can be written as $\eta_{0}+d \psi$. So the convex min cut program written in the form (3.35) remains unchanged. The rest of the proof goes through as before.

\section{The homotopy and homology problems}

In this section we discuss minimal-area metrics on a compact Riemann surface $M$, possibly with boundary. After fixing some notation we state the closed string field theory minimal-area problem as a convex program. This program is not amenable to numerical solution as stated because it involves a functional set of nonlocal constraints: the lengths of an infinite number of homotopically nontrivial closed curves are constrained, and each length constraint imposes nonlocal conditions on the metric. The minimal area problem can be modified to consider constraints on curves in a set of homotopy classes, with different length conditions on the various classes.

We examine then a more significant modification where we work with homology classes of curves. This time we consider a collection of non-trivial integral homology 1-cycles $C_{\alpha} \in H_{1}(M, \mathbb{Z})$, with 
$\alpha \in J$ an index labeling the cycles. We aim to minimize the total area of $M$ subject to the constraint that, for each $\alpha$, all representatives of $C_{\alpha}$ have length at least $\ell_{\alpha}$, where $\ell_{\alpha}>0$ is a constant. In subsection 4.2 we write this as a convex program. This program, just like the homotopy one, is not very practical as it also involves a functional constraint set. Therefore, in subsection 4.4 we use calibrations and the max flow-min cut theorem to rewrite the problem as a convex program with a finite number of local constraints. This form of the program allows one to obtain rigorous upper bounds on the minimal area and is also practical for numerical minimization.

We restrict ourselves in this paper to homology classes of closed curves, both for the sake of concreteness and because this is the case relevant to closed string field theory. Our considerations, however, carry over almost without modification to open curves, with the homology class defined relative to the endpoints of the curve. More generally, one can consider classes defined relative to other sets, such as the boundary of $M$; this requires imposing appropriate boundary conditions on the calibrations $u^{\alpha}$ and scalar fields $\varphi^{\alpha}$ defined below and in the following section.

\subsection{Notation}

Let us fix some notation to get started. We denote by $g_{\mu \nu}^{0}$ a fiducial conformal metric on the Riemann surface $M$. This means that for any local complex coordinate $z=x^{1}+i x^{2}$ on the surface, the fiducial metric takes the form

$$
d s^{2}=2 g_{z \bar{z}}^{0} d z d \bar{z}=2 g_{z \bar{z}}^{0}\left(\left(d x^{1}\right)^{2}+\left(d x^{2}\right)^{2}\right) .
$$

The conformal metrics $g_{\mu \nu}$ on $M$ are in one-to-one correspondence with functions $\Omega \geq 0$ on $M$ via

$$
g_{\mu \nu}=\Omega g_{\mu \nu}^{0}
$$

We will also write

$$
\Omega=\rho^{2},
$$

where $\rho$ is a non-negative function on $M:^{7}$

$$
g_{\mu \nu}=\rho^{2} g_{\mu \nu}^{0} .
$$

Since the relation between $\Omega$ and $\rho$ is non-linear, it does not automatically preserve convexity properties. Given that convexity plays a central role in our analysis, we will take care in each instance to specify whether we are using $\Omega$ or $\rho$ as our variable.

The area of $M$ is

$$
\operatorname{area}(M)=\int_{M} d^{2} x \sqrt{g}=\int_{M} d^{2} x \sqrt{g^{0}} \Omega=\int_{M} d^{2} \sqrt{g^{0}} \rho^{2}
$$

We also write

$$
\operatorname{area}(M)=\int_{M} \omega=\int_{M} \omega_{0} \Omega=\int_{M} \omega_{0} \rho^{2}
$$

where we define the volume form $\omega$ and the fiducial volume form $\omega_{0}$ as

$$
\omega=d^{2} x \sqrt{g}, \quad \omega_{0}=d^{2} x \sqrt{g_{0}} .
$$

\footnotetext{
${ }^{7}$ In the literature on Riemann surfaces, the metric is often written $d s^{2}=\rho^{2} d z d \bar{z}$. The reader familiar with that literature should be aware that our use of $\rho$ is different.
} 
Consider now the lengths of curves on the surface. Let $\gamma$ be a closed curve defined by a map $x^{\mu}(t):[0,1] \rightarrow M$. We then have

$$
\begin{aligned}
\operatorname{length}(\gamma) & :=\int_{0}^{1} d t\left|\dot{x}_{i}(t)\right|=\int_{0}^{1} d t \sqrt{g_{\mu \nu} \dot{x}^{\mu} \dot{x}^{\nu}} \\
& =\int_{0}^{1} d t \sqrt{\Omega g_{\mu \nu}^{0} \dot{x}^{\mu} \dot{x}^{\nu}}=\int_{0}^{1} d t \sqrt{\Omega}|\dot{x}|_{0}=\int_{0}^{1} d t \rho|\dot{x}|_{0}
\end{aligned}
$$

with $t$ derivatives denoted by dots, $|\cdot|$ denoting norm in the metric $g$ and $|\cdot|_{0}$ denoting norm in the fiducial metric $g_{0}$ (see Appendix A for more details on notation). For brevity we will write, symbolically,

$$
\operatorname{length}(\gamma)=\int_{\gamma} \sqrt{\Omega}|\dot{x}|_{0} .
$$

We can also consider lengths of representatives of non-trivial homology 1-cycles. Such a representative $m$ is a set of $k$ closed oriented curves $\gamma_{i}(m)$, with $i=1, \ldots, k$. We parameterize each of them with $t \in[0,1]$ using maps $x_{i}^{\mu}(t):[0,1] \rightarrow M$. The length of $m$ is then defined as

$$
\operatorname{length}(m):=\sum_{i=1}^{k} \int_{0}^{1} d t\left|\dot{x}_{i}(t)\right|=\sum_{i=1}^{k} \int_{0}^{1} d t \sqrt{\Omega}\left|\dot{x}_{i}\right|_{0} .
$$

For brevity we will write, symbolically

$$
\text { length }(m)=\int_{m} \sqrt{\Omega}|\dot{x}|_{0} .
$$

Here we are adding lengths regardless of orientation of the curves. If a representative contains both a curve $\gamma$ and the oppositely oriented curve $-\gamma$, which cancel in homology, the length functional adds the two lengths.

\subsection{Homotopic minimal-area programs}

The closed string field theory minimal-area problem (MAP) stated in the introduction and reviewed in section 2 is now stated as a convex program. We let $\ell_{s}$ denote the systole and $\Gamma$ the set of non-contractible closed curves on $M$, and write

$$
\begin{array}{ll}
\text { Closed string field theory MAP: } & \text { Minimize } \int_{M} \omega_{0} \Omega \text { over } \Omega \geq 0 \text { (function) } \\
& \text { subject to } \quad \ell_{s}-\int_{\gamma} \sqrt{\Omega}|\dot{x}|_{0} \leq 0, \forall \gamma \in \Gamma .
\end{array}
$$

The notation here means that $\Omega \geq 0$ is an implicit constraint: it defines the domain on which the objective and other constraint functions are defined. We have allowed the scale factor $\Omega$ to vanish, since this may occur at points on $M$ on the minimal-area metric (e.g. if it has conical singularities). The other constraint is explicit:

$$
\ell_{s}-\int_{\gamma} \sqrt{\Omega}|\dot{x}|_{0} \leq 0
$$

In a well-defined convex program, the objective and all explicit constraint functions must be convex on the domain defined by the implicit constraints, which must itself be a convex subset of an affine space. We can check that (4.11) is indeed a convex program: The domain, consisting of non-negative 
functions $\Omega$ on $M$, is clearly convex. The objective is a linear functional of $\Omega$ and therefore convex. And, for each curve $\gamma$, the constraint function $\ell_{s}-\int_{\gamma} \sqrt{\Omega}|\dot{x}|_{0}$, is an affine functional, with positive coefficients, of $-\sqrt{\Omega}$, which is itself a convex function of $\Omega$ for $\Omega \geq 0$. This will be discussed more explicitly below in the context of the homology version.

We can also write the program in terms of $\rho$ :

$$
\begin{aligned}
& \text { Minimize } \int_{M} \omega_{0} \rho^{2} \quad \text { over } \rho \geq 0 \text { (function) } \\
& \text { subject to } \ell_{s}-\int_{\gamma} \rho|\dot{x}|_{0} \leq 0, \forall \gamma \in \Gamma .
\end{aligned}
$$

This is also a convex program: The domain is again the set of non-negative functions on $M$, the objective is a convex functional, and the constraint function is an affine, hence convex, functional. (See [3] for further discussion.) Since the objective in (4.13) is strictly convex, this way of writing the program has the advantage of showing that the solution $\rho^{*}$ is unique, which implies that the solution $\Omega^{*}$ of $(4.11)$ is also unique.

A generalized version of this problem imposes different conditions on different homotopy classes of closed curves. Let $D_{\beta} \in \pi_{1}(M)$, with $\beta \in K$ an index labeling the various homotopy classes. To the curves on $D_{\beta}$ we associate the length constraint $\ell_{\beta}$. We then have

$$
\begin{array}{ll}
\text { Homotopy MAP: } & \text { Minimize } \int_{M} \omega_{0} \Omega \text { over } \Omega \geq 0 \text { (function) } \\
& \text { subject to } \quad \ell_{\beta}-\int_{\gamma} \sqrt{\Omega}|\dot{x}|_{0} \leq 0, \quad \forall \gamma \in D_{\beta}, \quad \forall \beta \in K .
\end{array}
$$

\subsection{Homological minimal-area program}

Again, let $C_{\alpha}$ with $\alpha \in J$ be a set of non-trivial homology cycles on $M$. We look for the minimal area (conformal) metric under the condition that, for any $\alpha \in J$, the length of any representative of $C_{\alpha}$ be greater than or equal to $\ell_{\alpha}$. In analogy to the homotopy MAP above, we write

$$
\begin{array}{ll}
\text { Homology MAP: } & \text { Minimize } \int_{M} \omega_{0} \Omega \text { over } \Omega \geq 0 \text { (function) } \\
& \text { subject to } \ell_{\alpha}-\int_{m} \sqrt{\Omega}|\dot{x}|_{0} \leq 0, \forall m \in C_{\alpha}, \forall \alpha \in J .
\end{array}
$$

Let us make explicit the fact that we have a convex program by showing that by discretization we get a program of the form (3.3). Our variable $\Omega$ on the surface $M$ is the analog of the vector $y \in \mathbb{R}^{n}$ in (3.3). We think of $\Omega$ as a vector $\vec{\Omega} \in \mathbb{R}^{N}$, whose components are the values $\Omega[i]$ over a discretization of the surface $M$ into small plaquettes indexed by $i=1, \cdots N$, for some large value of $N$. The implicit constraint $\Omega[i] \geq 0$ for all $i$, indeed defines a convex subspace of the affine space $\mathbb{R}^{N}$ where $\vec{\Omega}$ lives.

The objective $f_{0}$ in (3.3) is now a sum of the discrete values $\Omega[i]$ multiplied by the quantity $\omega_{0}[i] \geq 0$ representing the value of $d^{2} x \sqrt{g^{0}}$ for the $i$-th plaquette:

$$
f_{0}(\vec{\Omega})=\sum_{i=1}^{N} \Omega(i) \omega_{0}(i) .
$$


Written this way, the objective is clearly an affine function of $\vec{\Omega}$, and thus it is convex. Consider now the constraint on the length. Take some fixed cycle $m$, parameterized as $y(t)$. The cycle will go through some plaquettes whose values of $i$ are in a set $S_{m}$. For each $i \in S_{m}$ there is positive number $\chi_{m}[i] \geq 0$ capturing the way the curve crosses the $i$-th plaquette so that the length constraint takes the form

$$
\ell_{\alpha}-\sum_{i \in S_{m}} \sqrt{\Omega[i]} \chi_{m}[i] \leq 0 .
$$

Since the function $(-\sqrt{x})$ is convex for $x \geq 0$, the left-side of this inequality, being a non-negatively weighted sum of convex functions, is a convex function of $\vec{\Omega}$ over the domain $\vec{\Omega} \geq 0$.

Note that even as we discretize the program (4.15), there are an infinite number of constraints, since there are an infinite number of curves whose length must be constrained. In the program each cycle is represented by a set $S_{m}$ and the values of the (continuous) coefficients $\chi_{m}[i]$. An infinite number of constraints is difficult to handle. Of course, one gets a finite number of constraints if one decides to constrain the length of a finite number of curves, but it is unclear when the chosen set of curves suffices to get a good approximation.

There is no need to discretize to ascertain convexity if we are willing to consider a generalized form of the standard program (3.3) where the variable $x$ is replaced by a function, both the objective and the inequality constraints are defined by convex functionals, and the equality constraints are affine functionals. Letting $\Omega$ denote the function variable such program reads

$$
\begin{array}{ll}
\text { Minimize } & F_{0}[\Omega] \quad \text { over } \Omega \in \mathcal{D} \\
\text { subject to } & F_{i}[\Omega] \leq 0 \quad i=1,2 \ldots, \\
& H_{j}[\Omega]=0 \quad j=1, \ldots .
\end{array}
$$

Here $F_{0}$ and $F_{i}$ are convex functionals over some domain $\mathcal{D}$ for the functions $\Omega$, where $\mathcal{D}$ is a convex subset of an affine space. Moreover, the $H_{j}$ are affine functionals. The affine space in our case is the space of all functions $\Omega$ and the convex subset is the set of all $\Omega \geq 0$. A functional $\Phi$ is said to be convex if for functions $\Omega_{1}$ and $\Omega_{2}$ in $\mathcal{D}$ we have

$$
\Phi\left[t \Omega_{1}+(1-t) \Omega_{2}\right] \leq t \Phi\left[\Omega_{1}\right]+(1-t) \Phi\left[\Omega_{2}\right], \quad t \in[0,1] .
$$

The area functional

$$
F_{0}[\Omega]=\int_{M} \omega_{0} \Omega,
$$

is manifestly an affine, and thus convex functional. We have no equality constraints. The inequality constraints are the length constraints. Applied to the cycle $m \in C_{\alpha}$ it takes the form

$$
F_{m}[\Omega]=\ell_{\alpha}-\int_{m} \sqrt{\Omega}|\dot{y}|_{0},
$$

and $F_{m}$ is quickly shown to be a convex functional using the convexity of $-\sqrt{x}$, for $x>0$. Each constraint is non-local on the surface $M$, involving the scale factor on curves that stretch along $M$. Moreover we really have a functional constraint set: each constraint is associated to a function, the embedding map that defines the cycle on the surface.

We can also write the homology program in terms of $\rho$ :

$$
\begin{aligned}
& \text { Minimize } \int_{M} \omega_{0} \rho^{2} \quad \text { over } \rho \geq 0 \text { (function) } \\
& \text { subject to } \ell_{\alpha}-\int_{m} \rho|\dot{x}|_{0} \leq 0, \forall m \in C_{\alpha}, \forall \alpha \in J .
\end{aligned}
$$


As for (4.13), in this form the objective is strictly convex, which shows that the solution $\rho^{*}$ is unique. This implies in turn that the program (4.15) also has a unique solution $\Omega^{*}$.

The advantage of the homology MAP over the homotopy one is that, as we will show in the next subsection, by using calibrations it can be converted into a program with a finite number of local constraints. Furthermore, as we will show in section 8 , the closed string field theory homotopy program can be written as a homology program by the trick of passing to a covering space.

\subsection{Reformulation as a local problem using calibrations}

In this subsection we will use the device of calibrations to reformulate the homological minimal-area problem (4.15) as a convex program with constraints that are almost entirely local on $M$. For this we will rely crucially on the max flow-min cut (MFMC) theorem. Calibrations and MFMC were described in subsection 3.3. Here we will specialize to the two-dimensional case and give further details.

We define a 1 -calibration on a manifold as a closed one-form $u$ obeying $|u| \leq 1$ everywhere:

$$
\text { Calibration: } d u=0,|u| \leq 1 \text {. }
$$

There is no boundary condition on $u$. Since $u$ is closed, its integral over a cycle in a homology class $C$ is independent of the representative on which it is integrated, so we write

$$
\int_{C} u
$$

for the period of the calibration. As is well known, by virtue of the constraint $|u| \leq 1$, this integral gives a lower bound for the length of any representative $m \in C$ :

$$
\left|\int_{C} u\right| \leq \text { length }(m) .
$$

To verify this we write $m$ as a set of curves $x_{i}^{\mu}(t)$. Since $m$ belongs to the homology class $C$ and the form $u$ is closed we have

$$
\int_{C} u=\int_{m} u=\sum_{i} \int_{0}^{1} d t u\left(\dot{x}_{i}(t)\right)=\sum_{i} \int_{0}^{1} d t\left\langle\hat{u}, \dot{x}_{i}(t)\right\rangle .
$$

Here the vector $\hat{u}$ is obtained by raising the index on the one-form $u$ with the help of the metric, and $\langle\cdot, \cdot\rangle$ is the inner product on the space of vectors (see Appendix A for notation and identities). Taking absolute values we have

$$
\left|\int_{C} u\right|=\left|\sum_{i} \int_{0}^{1} d t\left\langle\hat{u}, \dot{x}_{i}(t)\right\rangle\right| \leq \sum_{i}\left|\int_{0}^{1} d t\left\langle\hat{u}, \dot{x}_{i}(t)\right\rangle\right| \leq \sum_{i} \int_{0}^{1} d t\left|\left\langle\hat{u}, \dot{x}_{i}(t)\right\rangle\right| .
$$

The inequalities above are all saturated if the integrand $\left\langle\hat{u}, \dot{y}_{i}(t)\right\rangle$ is everywhere positive. Using the Schwarz inequality $\left|\left\langle v, v^{\prime}\right\rangle\right| \leq|v|\left|v^{\prime}\right|$ and $|\hat{u}|=|u| \leq 1$ we now get

$$
\left|\int_{C} u\right| \leq \sum_{i} \int_{0}^{1} d t|u|\left|\dot{x}_{i}(t)\right| \leq \int_{0}^{1} d t|\dot{x}(t)|=\text { length }(m),
$$


which is what we wanted to prove. It is also clear what we need for the length inequality to be saturated:

$$
\text { length }(m)=\left|\int_{C} u\right| \text { requires } \hat{u} \propto \dot{x} \text { and }|u|=1 \text { on } m \text {. }
$$

Furthermore, the sign of the proportionality between $\hat{u}$ and $\dot{x}$ should be constant on $m$.

We have seen that if there exists a calibration with period $\ell$ on $C$ then all representatives of $C$ have length at least $\ell$. Furthermore, if the minimal-length representative has length $\ell^{\prime} \geq \ell$, then, by the MFMC theorem, there exists a calibration $u^{\prime}$ with period $\ell^{\prime}$. Then $u:=\left(\ell / \ell^{\prime}\right) u^{\prime}$ is also a calibration, and has period $\ell$. In summary, there exists a calibration with period $\ell$ if and only if all representatives of $C$ have length at least $\ell$. In this way, we can replace the length condition on every representative of a cycle with the existence of a calibration of a given period. Except for the period condition, all conditions on a calibration $u$ are local on the surface.

For each cycle $C_{\alpha}, \alpha \in J$, on which we impose the length constraint, we demand the existence of a corresponding calibration $u^{\alpha}$. The constraints on $u^{\alpha}$ are:

$$
d u^{\alpha}=0, \quad\left|u^{\alpha}\right| \leq 1, \quad \int_{m_{\alpha}} u^{\alpha}=\ell_{\alpha},
$$

where $m_{\alpha}$ is an arbitrary representative of $C_{\alpha}$. It is necessary to choose a representative since the period $\int_{C_{\alpha}} u^{\alpha}$ is not well-defined before the constraint $d u^{\alpha}=0$ is imposed. The first and third constraints are affine in the variable $u^{\alpha}$ and don't depend on the scale factor $\Omega$. We can rewrite the second constraint as follows:

$$
\left|u^{\alpha}\right|_{0}^{2}-\Omega \leq 0
$$

As written, the constraint (4.31) is convex in $u^{\alpha}$ and affine in $\Omega$. Viewed as $\Omega \geq\left|u^{\alpha}\right|_{0}^{2}$, we see that the fiducial norm of the calibration "props up" the scale factor $\Omega$. We can now drop the implicit constraint $\Omega \geq 0$ that we had in our first program (4.15), which simplifies the subsequent analysis a bit. We now have the following convex program:

$$
\begin{aligned}
& \text { Primal MAP v1: Minimize } \int_{M} \omega_{0} \Omega \text { over } \Omega \text { (function), } u^{\alpha} \text { (one-forms) } \\
& \text { subject to } \quad\left|u^{\alpha}\right|_{0}^{2}-\Omega \leq 0 \text {, } \\
& d u^{\alpha}=0, \\
& \ell_{\alpha}-\int_{m_{\alpha}} u^{\alpha}=0, \forall \alpha \in J
\end{aligned}
$$

There is no boundary condition on $u^{\alpha}$. Any feasible $u^{\alpha}, \Omega$ provides a rigorous upper bound on the value of the minimum. We call this program "primal" because in the next section we will derive a second minimal-area program, related to this one by Lagrangian duality, which we will call the dual minimal-area program.

As for the homotopy and homology programs, we can make the change of variables from $\Omega$ to $\rho$ 
in (4.32), and the program remains convex:

$$
\begin{aligned}
& \text { Minimize } \int_{M} \omega_{0} \rho^{2} \text { over } \rho \text { (function), } u^{\alpha} \text { (one-forms) } \\
& \text { subject to } \quad\left|u^{\alpha}\right|_{0}-\rho \leq 0 \text {, } \\
& d u^{\alpha}=0, \\
& \ell_{\alpha}-\int_{m_{\alpha}} u^{\alpha}=0, \forall \alpha \in J
\end{aligned}
$$

Again, since the objective is strictly convex in $\rho$, it is clear that $\rho$ takes a unique value $\rho^{*}$ in the solution. On the other hand, the one-forms $u^{\alpha}$ do not appear in the objective, so they are not necessarily unique. To see the non-uniqueness we solve for $\rho$ :

$$
\rho=\max _{\alpha}\left|u^{\alpha}\right|_{0}, \quad \text { at every point on } M .
$$

If we use this solution, the program is now:

$$
\begin{array}{ll}
\text { Minimize } & \int_{M} \omega_{0} \max _{\alpha}\left|u^{\alpha}\right|_{0}^{2} \text { over } u^{\alpha} \text { (one-forms) } \\
\text { subject to } & d u^{\alpha}=0 \\
& \ell_{\alpha}-\int_{m_{\alpha}} u^{\alpha}=0, \forall \alpha \in J .
\end{array}
$$

The objective is convex in the space of $u$ 's: it is the pointwise maximum of a set of convex functions. While (4.35) is simpler-looking, we have found it easier in practice to work with the form (4.32). Returning to the non-uniqueness of $u^{\alpha}$, it is clear from (4.34) that we can change any calibration $u^{\alpha}$ over a region $R$ where $\left|u^{\alpha}\right|_{0}<\rho$ without changing the value of the objective while keeping $u^{\alpha}$ closed and its periods unchanged. For this we simply let $u^{\alpha} \rightarrow u^{\alpha}+d \chi$, where $\chi$ is a smooth function, chosen to be sufficiently small and supported only inside the region $R$.

The program (4.32) still contains a finite number of non-local constraints, namely the period conditions $\int_{m_{\alpha}} u^{\alpha}=\ell_{\alpha}$, as compared to the functional infinity of constraints appearing in (4.15). By using a basis of real closed one-forms, however, we can rewrite these constraints as algebraic conditions, eliminating the need to do integrals on $M$ to check that the constraints are satisfied. Moreover, the algebraic conditions can sometimes be solved directly. Let $\omega^{i}$ be a real basis for $H^{1}(M),{ }^{8}$ closed one-forms with periods:

$$
\omega_{\alpha}^{i}:=\int_{C_{\alpha}} \omega^{i}
$$

(Do not confuse the basis one forms $\omega^{i}$ with the volume forms $\omega$ and $\omega_{0}$.) We can then write $u^{\alpha}$ as as a linear combination of the basis one-forms plus an exact one-form $d \phi^{\alpha}$ where $\phi^{\alpha}$ is a function on $M$ :

$$
u^{\alpha}=\sum_{i} c_{i}^{\alpha} \omega^{i}+d \phi^{\alpha}
$$

The constants $c_{i}^{\alpha}$ are constrained by the period conditions $\int_{C_{\alpha}} u^{\alpha}=\ell_{\alpha}$ :

$$
\sum_{i} c_{i}^{\alpha} \omega_{\alpha}^{i}=\ell_{\alpha}
$$

\footnotetext{
${ }^{8}$ We work with real one-forms throughout, as the calibrations are defined to be real.
} 
If the number of constraints here (equal to the number of homology classes) is no more than the number of independent cycles, then by an appropriate choice of one-forms $\omega^{i}$ the constraint (4.38) can be solved directly. Having replaced the calibrations by the constants $c_{i}^{\alpha}$ and the functions $\phi^{\alpha}$, where the zero mode of $\phi^{\alpha}$ drops out, the program reads:

Primal MAP v2: Minimize $\int_{M} \omega_{0} \Omega$ over $c_{i}^{\alpha}$ (constants), $\Omega, \phi^{\alpha}$ (functions)

$$
\begin{aligned}
\text { subject to }\left|\sum_{i} c_{i}^{\alpha} \omega^{i}+d \phi^{\alpha}\right|_{0}^{2}-\Omega & \leq 0, \\
\ell_{\alpha}-\sum_{i} c_{i}^{\alpha} \omega_{\alpha}^{i} & =0, \quad \forall \alpha \in J .
\end{aligned}
$$

We will see in [22] that with a suitable discretization the program (4.39) is straightforward to solve numerically.

\section{An equivalent dual problem}

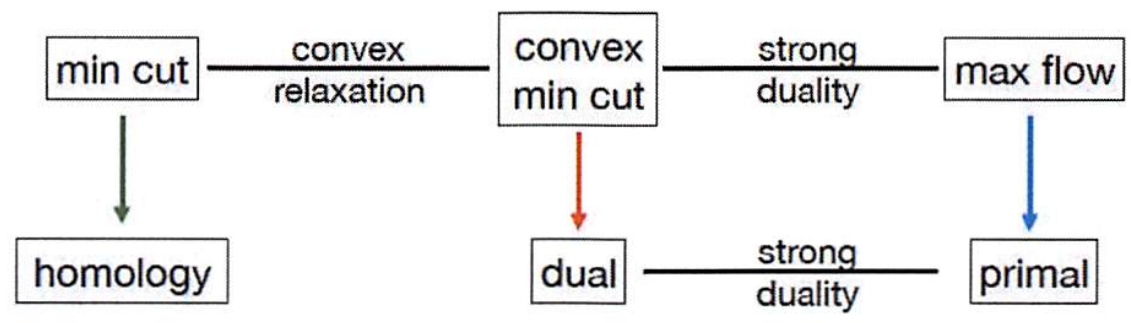

Figure 6: Illustration of the relations among the various programs in this paper. The top line gives the programs for finding the minimal length in a given homology class. Min cut is given in (3.36), convex min cut in (3.35), and max flow in (3.29). The bottom line gives the minimal-area programs. The homology minimal area program (MAP) is given in subsection 4.3 , the dual program in 5.1 , and the primal in 4.4. Each one is connected by an arrow to the minimal-length program employed in its derivation. The green arrow (left) is direct. The blue arrow (right) requires a short argument, appearing between (4.29) and (4.30). The derivations of the dual MAP using convex min cut (red arrow) are given in subsections 5.2.1 and 5.2.2.

In this section we will derive another convex program that solves the homological minimal-area problem described in subsection 4.3. Like the primal minimal-area program (4.32), it involves only local variables and constraints, and is amenable to numerical solution. Perhaps surprisingly, it is a maximization problem and does not involve the metric at all. It involves instead a function $\varphi^{\alpha}$ and a constant $\nu^{\alpha}$ for each homology class $C_{\alpha}$ constrained with length parameter $\ell_{\alpha}$. However, as we will explain in section 7.1, it is straightforward to use a solution to this program to read off both the minimal-area metric and the curves saturating the length constraint (if any) in each class. We will call this program, in its various simply related versions, the dual minimal-area program, since its derivation involves a dualization, starting either from the homology minimal-area program (4.15) or the so-called primal one (4.32). The program will be stated and briefly discussed in subsection 5.1, and then derived in three different ways in 5.2. Figure 6 illustrates the relations among the various programs in this paper. 


\subsection{Statement}

We start with the same data defining the homological minimal-area problem (4.15) described in the previous section: a Riemann surface $M$, possibly with boundary, equipped with a fiducial metric $g_{\mu \nu}^{0}$, a set of non-trivial integral homology classes $C_{\alpha} \in H_{1}(M, \mathbb{Z})$, and for each one a positive number $\ell_{\alpha}$. We then require that, for all $\alpha$, the length of every representative of $C_{\alpha}$ is at least $\ell_{\alpha}$, and we wish to find the smallest area among metrics in the Weyl class of $g_{\mu \nu}^{0}$ satisfying this constraint.

Let $\tilde{C}^{\alpha} \in H^{1}(M)$ be the 1-cohomology class that is Poincaré dual to $C_{\alpha}$ or, in the presence of a boundary, the Poincaré-Lefschetz dual (see above (3.49)). We will denote by $\eta^{\alpha}$ a general representative of $\tilde{C}^{\alpha}$. We claim that the above problem is solved by the following convex program, henceforth called the first dual version:

$$
\begin{aligned}
& \text { Dual MAP v1: Maximize } 2 \sum_{\alpha} \nu^{\alpha} \ell_{\alpha}-\int_{M} \omega_{0}\left(\sum_{\alpha} \nu^{\alpha}\left|\eta^{\alpha}\right|_{0}\right)^{2} \text { over } \nu^{\alpha} \text { (constants), } \eta^{\alpha} \in \tilde{C}^{\alpha} \\
& \text { subject to } \quad \nu^{\alpha} \geq 0, \quad \forall \alpha \in J \text {. }
\end{aligned}
$$

Here $\omega_{0}=\sqrt{g^{0}} d^{2} x$ and $|\cdot|_{0}$ are, respectively, the area form and norm with respect to the fiducial metric. The program in fact is independent of the choice of fiducial metric within a given Weyl class, since under a Weyl transformation $g_{\mu \nu}^{0} \rightarrow \hat{\rho}^{2} g_{\mu \nu}^{0}$, the area form and norm transform as $\omega_{0} \rightarrow \hat{\rho}^{2} \omega_{0}$ and $|\cdot|_{0} \rightarrow \hat{\rho}^{-1}|\cdot|_{0}$, so the objective is unchanged. It is a straightforward exercise to show that the objective is a concave functional of $\nu^{\alpha}$ and $\varphi^{\alpha}$, and the constraint functions are linear, hence concave, so (5.1) is indeed a convex program. We will derive this program by three different routes in subsection 5.2.

A solution to (5.1) gives us not only the value of the minimal area but also the minimizing metric and curves saturating the length constraint. Given a solution $\left(\nu^{\alpha *}, \eta^{\alpha *}\right)$, the metric is

$$
g_{\mu \nu}=\Omega^{*} g_{\mu \nu}^{0}, \quad \Omega^{*}=\left(\rho^{*}\right)^{2}, \quad \rho^{*}=\sum_{\alpha} \nu^{\alpha *}\left|\eta^{\alpha *}\right|_{0}
$$

Furthermore, for any $\alpha$ such that $\nu^{\alpha *}>0$, the level sets $m_{\eta^{\alpha *}}(t)$ defined in (3.44) saturate the length constraint. We will derive these facts in subsection 7.1.

We can write the program (5.1) in a more concrete form by choosing, for each $\alpha$, a fiducial representative $\eta_{0}^{\alpha}$ of $\tilde{C}^{\alpha}$. Then any $\eta \in \tilde{C}^{\alpha}$ can be written $\eta^{\alpha}=\eta_{0}^{\alpha}+d \psi^{\alpha}$ for some function $\psi^{\alpha}$ satisfying $\left.\psi^{\alpha}\right|_{\partial M}=0$. Defining $\varphi^{\alpha}$ by

$$
\varphi^{\alpha}:=\nu^{\alpha} \psi^{\alpha}
$$

and recalling that $\nu^{\alpha} \geq 0$, we have

$$
\nu^{\alpha}\left|\eta^{\alpha}\right|_{0}=\left|\nu^{\alpha} \eta_{0}^{\alpha}+d \varphi^{\alpha}\right|_{0}
$$

This change of variables puts the program into the following form:

Dual MAP v2:

$$
\text { Maximize } 2 \sum_{\alpha} \nu^{\alpha} \ell_{\alpha}-\int_{M} \omega_{0}\left(\sum_{\alpha}\left|\nu^{\alpha} \eta_{0}^{\alpha}+d \varphi^{\alpha}\right|_{0}\right)^{2} \text { over } \nu^{\alpha} \text { (constants), } \varphi^{\alpha} \text { (functions) }
$$

subject to

$$
\left.\varphi^{\alpha}\right|_{\partial M}=0 \quad \forall \alpha \in J .
$$


In going from (5.1) to (5.5), we actually dropped two constraints. First, according to (5.3), $\nu^{\alpha}=0$ implies $\varphi^{\alpha}=0$, but in (5.5) we do not impose this constraint. However, if $\nu^{\alpha}=0$, then the objective will in any case be maximized by setting $d \varphi^{\alpha}=0$. If $M$ has a boundary, the boundary condition then implies $\varphi^{\alpha}=0$. If not, the program (5.5) is invariant under $\varphi^{\alpha} \rightarrow \varphi^{\alpha}+$ constant, so we are free to set $\varphi^{\alpha}=0$. Second, we dropped the constraint $\nu^{\alpha} \geq 0$. However, if $\nu^{\alpha}<0$ then the objective will be increased by taking $\nu^{\alpha} \rightarrow-\nu^{\alpha}$ and $\varphi^{\alpha} \rightarrow-\varphi^{\alpha}$. So neither of these "relaxations" changes the solution, and (5.5) is indeed equivalent to (5.1).

We will now write (5.5) in an even more concrete third form by making a specific choice of fiducial one-forms $\eta_{0}^{\alpha}$, namely the delta-function "bump" forms defined in (3.38). Choose a representative $m_{\alpha}$ of each homology class $C_{\alpha}$ and set $\eta_{0}^{\alpha}=\eta_{m_{\alpha}}$, which equals $\delta\left(x^{2}\right) d x^{2}$ in a local coordinate system in which $m_{\alpha}$ is at $x^{2}=0$. The second term in the objective (5.5) contains in the integrand the square of a sum of norms of one-forms. It therefore contains the square of a delta function, giving a divergent integral, unless $d \varphi^{\alpha}$ has a compensating delta function,

$$
d \varphi^{\alpha}=-\nu^{\alpha} \delta\left(x^{2}\right) d x^{2}+\text { regular } .
$$

Thus the objective is finite only if $\varphi^{\alpha}$ jumps by $-\nu^{\alpha}$ along $m_{\alpha}$. We can make the constraint $\left.\Delta \varphi^{\alpha}\right|_{m_{\alpha}}=-\nu^{\alpha}$ (which is linear in $\varphi^{\alpha}$ and $\nu^{\alpha}$ ) explicit. Away from $m_{\alpha}, \eta_{m_{\alpha}}$ vanishes. In this form, the program becomes

\section{Dual MAP v3:}

$$
\begin{array}{ll}
\text { Maximize } & 2 \sum_{\alpha} \nu^{\alpha} \ell_{\alpha}-\int_{M^{\prime}} \omega_{0}\left(\sum_{\alpha}\left|d \varphi^{\alpha}\right|_{0}\right)^{2} \text { over } \nu^{\alpha} \text { (constants), } \varphi^{\alpha} \text { (functions) } \\
\text { subject to } & \left.\Delta \varphi^{\alpha}\right|_{m_{\alpha}}=-\nu^{\alpha}, \\
& \left.\varphi^{\alpha}\right|_{\partial M}=0, \quad \forall \alpha \in J .
\end{array}
$$

Here $M^{\prime}$ is the manifold $M$ with the chosen representative curves $m_{\alpha}$ removed:

$$
M^{\prime}=M \backslash \cup_{\alpha \in J} m_{\alpha} .
$$

This is just a way to tell us that in calculating the objective with our necessarily discontinuous $\varphi^{\alpha}$ there is no delta-function contribution in $d \varphi^{\alpha}$ on $m_{\alpha}$. Note also that we don't need to be careful about the orientation of $m_{\alpha}$ or the sign of the jump, since the objective is invariant under $\varphi^{\alpha} \rightarrow-\varphi^{\alpha}$.

The program (5.7) is the final form of our dual program. In trying to maximize the objective the first term tries to make $\nu^{\alpha}$ large; however, a non-zero jump forces $\varphi^{\alpha}$ to have a non-zero gradient somewhere, which makes the second term more negative. The former is linear while the latter is quadratic, so we expect a maximum to exist. Note that the second term in the objective is a little unusual: it is the square of a sum of norms rather than the more familiar (to physicists) sum of norm-squared terms. On the maximum not all homology classes may be active: $\nu^{\alpha}$ and $\varphi^{\alpha}$ may vanish for some $\alpha$.

An interesting special case occurs when, for some $\alpha$, a subset $m_{\alpha}^{\prime}$ of the full boundary $\partial M$ is a representative of the class $C_{\alpha}$. In this case the representative $m_{\alpha}$ where $\varphi^{\alpha}$ jumps can be pushed all the way until it reaches the curve $m_{\alpha}^{\prime}$ in the boundary. Since the value of $\varphi^{\alpha}$ at the boundary is supposed to be zero, placing the discontinuity at $m_{\alpha}^{\prime}$ effectively sets the value of $\varphi^{\alpha}$ on $m_{\alpha}^{\prime}$ equal to $\nu^{\alpha}$. The value of $\varphi^{\alpha}$ on the rest of the $\partial M$ remains zero. In other words, for that $\alpha$ we replace the 
constraints in (5.7) by the following boundary conditions:

$$
\left.\varphi^{\alpha}\right|_{\partial M \backslash m_{\alpha}^{\prime}}=0,\left.\quad \varphi^{\alpha}\right|_{m_{\alpha}^{\prime}}=\nu^{\alpha} .
$$

Like the primal (4.39), the dual program (5.7) is straightforward to solve numerically. Furthermore, any trial values for $\nu^{\alpha}, \varphi^{\alpha}$ give a rigorous lower bound on the maximum. Using both the primal (4.39) and the dual (5.7), we can thus bound the minimum area both above and below. As in program Dual MAP v1, a solution $\left(\nu^{\alpha *}, d \varphi^{\alpha *}\right)$ of Dual MAP v3 also gives us the minimizing metric $\rho^{*}$

$$
\rho^{*}=\sum_{\alpha}\left|d \varphi^{\alpha *}\right|_{0}
$$

We noted that the solution of the primal problem was not unique because the calibrations $u^{\alpha}$ are ambiguous in the regions where their fiducial norm squared does not equal the Weyl factor $\Omega$ and they do not appear explicitly in the objective. For the dual program (5.7), one can find fine-tuned examples for which the solution is not unique (such as the square torus; see section 6.2). We believe, however, that the solution is generically unique, up to shifts of the functions $\varphi^{\alpha}$ by constants. This intuition is supported by the identification of saturating geodesics from the functions $\varphi^{\alpha}$, to be discussed in section 7.1.

Solving for the $\nu$ 's: The dual programs presented above involve maximization over some nonnegative constants $\nu^{\alpha}$ and some one-forms, or functions, depending on the version. As mentioned below (5.2), on the solution, $\nu^{\alpha *}>0$ for any $\alpha$ such that the length constraint in that homology class is active, i.e. such that there exist representatives $m \in C_{\alpha}$ saturating the length constraint. If we happen to know which homology classes are active, then we can ignore the $\nu^{\alpha} \geq 0$ constraint for those classes, and simply drop the other ones altogether from the program. This makes it possible to perform the maximization over the $\nu$ 's. We will do so, although it is not clear if the resulting objective, while simple looking, is particularly useful. Take, for example, version 1 of the dual program (5.1), whose objective $O_{1}$ is

$$
O_{1}=2 \sum_{\alpha} \nu^{\alpha} \ell_{\alpha}-\sum_{\alpha, \beta} \nu^{\alpha} \nu^{\beta} M_{\alpha \beta}, \quad M_{\alpha \beta}:=\int_{M} \omega_{0}\left|\eta^{\alpha}\right|_{0}\left|\eta^{\alpha}\right|_{0} \geq 0
$$

Using vector notation $\nu=\left(\nu^{1}, \ldots\right), \ell=\left(\ell_{1}, \cdots\right)$ and letting $\mathbf{M}$ denote the symmetric matrix with components $M_{\alpha \beta} \geq 0$, the objective is

$$
O_{1}=2 \nu^{T} \ell-\nu^{T} \mathrm{M} \nu .
$$

Generically the matrix $\mathbf{M}$ is invertible (otherwise a slightly more involved treatment is required). Maximization over $\nu$ leads to $\ell=\mathbf{M} \nu$ and therefore $\nu=\mathbf{M}^{-1} \ell$. Putting it back into the objective we get the new objective

$$
O_{1}^{\prime}=\ell^{T} \mathbf{M}^{-1} \ell
$$

It remains, of course to maximize $O_{1}^{\prime}$ over the one-forms $\eta$ entering into the definition of the $\mathbf{M}$ matrix elements. It is noteworthy that the Schwarz inequality on the surface implies that

$$
M_{\alpha \beta} \leq \sqrt{M_{\alpha \alpha} M_{\beta \beta}},
$$

where repeated indices are not summed. Note that this result can easily be adapted for version 3 of the dual program. In this case one would define tilde functions $\varphi^{\alpha}=\nu^{\alpha} \tilde{\varphi}^{\alpha}$ with unit discontinuities $\Delta \tilde{\varphi}^{\alpha}=-1$ and a similar writing of the objective is possible. 


\subsection{Derivations}

In this subsection, we will derive the dual minimal-area program. We will in fact present three different derivations. Each of these derivations illuminates the relation between the dual program and the original homological minimal-area program (4.15). This is valuable given that, at first blush, these two problems appear to be totally unrelated. The derivations also give insight into the nature of the solutions. Lagrangian duality plays a central role in each one, justifying the name "dual minimal-area program". The first derivation is perhaps the most intuitive, the second is the shortest, and the last one highlights the relationship between the dual and the primal minimal-area program (4.32).

\subsubsection{Maximin}

In proving the MFMC theorem, we established the equality (3.48) of the min cut and convex min cut programs. In our present context, where $M$ is two-dimensional, we write

$$
\inf _{m \in C} \operatorname{length}(m)=\inf _{\eta \in \tilde{C}} \int_{M} \omega|\eta|
$$

Given the homological minimal area problem, a given metric $\rho$ is feasible if and only if, for all $\alpha$, every representative $m_{\alpha}$ of the class $C_{\alpha}$ has length at least $\ell_{\alpha}$. Using (5.15), this is equivalent to the condition that, for all $\alpha$ and all $\eta^{\alpha} \in \tilde{C}^{\alpha}$,

$$
\int_{M} \omega\left|\eta^{\alpha}\right| \geq \ell_{\alpha}
$$

Making the dependence on $\rho$ explicit by substituting $\omega=\omega_{0} \rho^{2}$ and $\left|\eta^{\alpha}\right|=\rho^{-1}\left|\eta^{\alpha}\right|_{0},(5.16)$ becomes

$$
\int_{M} \omega_{0} \rho\left|\eta^{\alpha}\right|_{0} \geq \ell_{\alpha}
$$

This provides an equivalent reformulation of the length conditions in the homological minimal area problem (4.15).

Any given $\eta^{\alpha} \in \tilde{C}^{\alpha}$ imposes, via (5.17), a constraint on $\rho$. Rather than considering all of these constraints at the same time, we will pick just one $\eta^{\alpha}$ for each $\alpha$, and minimize the area $\int_{M} \omega_{0} \rho^{2}$ subject only to the corresponding finite number of constraints. The resulting metric only gives a lower bound on the area, for (5.17) may be violated for some other choice of $\eta^{\alpha}$ s. However, we will argue that, among those choices, the metric giving the greatest lower bound in fact obeys all the constraints in (5.17). The proof of the last statement hinges crucially on the convexity of the space of $\eta^{\alpha}$; it would not work if we tried to follow the same procedure using instead the homology representatives $m_{\alpha}$, which do not form a convex set.

Let us then fix an $\eta^{\alpha}$ from each class $\tilde{C}^{\alpha}$. Denote this set $\eta=\left(\eta^{\alpha}\right)_{\alpha \in J}$. The following program minimizes the area over $\rho$ subject to the constraints (5.17):

$$
\begin{array}{ll}
\text { Minimize } & \int_{M} \omega_{0} \rho^{2} \text { over } \rho \text { (function) } \\
\text { subject to } & \ell_{\alpha}-\int_{M} \omega_{0} \rho\left|\eta^{\alpha}\right|_{0} \leq 0 \quad \forall \alpha \in J .
\end{array}
$$


Let $\rho[\eta]$ denote the minimizing metric and $A[\boldsymbol{\eta}]$ its area. (According to the notation of subsection 3.1 , we should write these as $\rho^{*}[\eta]$ and $A^{*}[\eta]$, but to avoid cluttering the notation we drop the stars.) We now dualize this program. Associated to the length constraints we introduce Lagrange multipliers $\nu^{\alpha} \geq 0$, assembled into a vector $\nu$. The Lagrangian functional is

$$
L=2 \sum_{\alpha} \nu^{\alpha} \ell_{\alpha}+\int_{M} \omega_{0}\left(\rho^{2}-2 \rho \sum_{\alpha} \nu^{\alpha}\left|\eta^{\alpha}\right|_{0}\right) .
$$

We can easily minimize $L$ pointwise with respect to $\rho$. The minimum is at

$$
\rho=\sum_{\alpha} \nu^{\alpha}\left|\eta^{\alpha}\right|_{0}
$$

and we are left with the following program:

$$
\begin{array}{ll}
\text { Maximize } & g_{\eta}[\nu]:=2 \sum_{\alpha} \nu^{\alpha} \ell_{\alpha}-\int_{M} \omega_{0}\left(\sum_{\alpha} \nu^{\alpha}\left|\eta^{\alpha}\right|_{0}\right)^{2} \text { over } \nu \text { (constants) } \\
\text { subject to } \quad \nu^{\alpha} \geq 0, \quad \forall \alpha \in J .
\end{array}
$$

Strong duality holds for the convex program (5.18): we can always make $\rho$ large enough that the constraint is strictly obeyed, so Slater's condition is satisfied. Let $\nu[\eta]$ be the maximizing value of $\nu$ in the program (5.21). According to (3.16), the solution to (5.18) is given by (5.20) with $\nu$ set to $\nu[\eta]$ :

$$
\rho[\eta]=\sum_{\alpha} \nu^{\alpha}[\eta]\left|\eta^{\alpha}\right|_{0}
$$

Let $A^{*}$ be the minimal area subject to the constraint (5.17) for all possible choices of $\eta$; this is the optimal value of the homology minimal-area program of section 4 . As we discussed above, for any given $\boldsymbol{\eta}$ we have

$$
A[\eta] \leq A^{*}
$$

so

$$
\sup _{\eta} A[\eta] \leq A^{*}
$$

Let $\eta^{*}$ be the maximizer of $A[\eta]$. We will show below that the corresponding metric $\rho\left[\eta^{*}\right]$ is feasible, i.e. obeys (5.17) for all $\eta$. Assuming this for the moment, we now find

$$
\sup _{\boldsymbol{\eta}} A[\eta]=A\left[\eta^{*}\right] \geq A^{*}
$$

because any feasible metric must have area greater than or equal to the minimum. Combining the last two inequalities gives

$$
\sup _{\eta} A[\eta]=A^{*}
$$

This shows that the primal minimal-area program, having $A^{*}$ as optimum, is equivalent to the program (5.21) supplemented by maximization over $\eta$. But such a program is in fact (5.1), thereby proven equivalent to the homological minimal-area program (4.15).

It remains to show that $\rho\left[\eta^{*}\right]$ is feasible. We will proceed by contradiction, showing that any $\eta$ for which $\rho[\eta]$ is infeasible does not maximize $A[\eta]$. Suppose that for some $\beta \in J$ and some $\hat{\eta}^{\beta} \in \tilde{C}^{\beta}$, the length condition (5.17) is violated:

$$
\int_{M} \omega_{0} \rho[\eta]\left|\hat{\eta}^{\beta}\right|_{0}<\ell_{\beta}
$$


We will construct an $\eta^{\prime}$ and a $\nu^{\prime}$ such that

$$
g_{\eta^{\prime}}\left[\nu^{\prime}\right]>g_{\eta}[\nu[\eta]]
$$

where $\nu[\eta]$ denotes the maximizing value of $\nu$ in the program (5.21). It will then follow that

$$
A\left[\boldsymbol{\eta}^{\prime}\right]=\sup _{\nu} g_{\boldsymbol{\eta}^{\prime}}[\boldsymbol{\nu}] \geq g_{\boldsymbol{\eta}^{\prime}}\left[\boldsymbol{\nu}^{\prime}\right]>g_{\boldsymbol{\eta}}[\boldsymbol{\nu}[\boldsymbol{\eta}]]=A[\boldsymbol{\eta}],
$$

showing that $\boldsymbol{\eta}$ is not maximal, and completing the proof.

It remains to construct an $\eta^{\prime}$ and a $\nu^{\prime}$ so that (5.28) holds. The two cases $\nu^{\beta}[\eta]>0$ and $\nu^{\beta}[\eta]=0$ require separate analyses. For $\nu^{\beta}[\eta]>0$, we know by complementary slackness (see (3.15)) that the corresponding constraint in (5.18) is saturated, i.e.

$$
\int_{M} \omega_{0} \rho[\eta]\left|\eta^{\beta}\right|_{0}=\ell_{\beta}
$$

We use the length-violating one-form $\hat{\eta}^{\beta} \in \tilde{C}$ to define $\boldsymbol{\eta}^{\prime}$ and $\boldsymbol{\nu}^{\prime}$ by

$$
\eta^{\alpha}=\eta^{\alpha} \quad \text { for } \alpha \neq \beta, \quad \eta^{\prime \beta}=(1-\epsilon) \eta^{\beta}+\epsilon \hat{\eta}^{\beta}, \quad \nu^{\prime \alpha}=\nu^{\alpha}[\eta] \text { for all } \alpha,
$$

where $0<\epsilon<1$. By the convexity of the norm we have

$$
\left|\eta^{\prime \beta}\right|_{0} \leq(1-\epsilon)\left|\eta^{\beta}\right|_{0}+\epsilon\left|\hat{\eta}^{\beta}\right|_{0}
$$

and therefore

$$
\sum_{\alpha} \nu^{\alpha}\left|\eta^{\alpha}\right|_{0} \leq \sum_{\alpha} \nu^{\alpha}[\eta]\left|\eta^{\alpha}\right|_{0}+\epsilon \nu^{\beta}[\eta]\left(\left|\hat{\eta}^{\beta}\right|_{0}-\left|\eta^{\beta}\right|_{0}\right)
$$

Squaring and integrating,

$$
\begin{aligned}
\int_{M} \omega_{0}\left(\sum_{\alpha} \nu^{\prime \alpha}\left|\eta^{\prime \alpha}\right|_{0}\right)^{2} & \leq \int_{M} \omega_{0}\left(\sum_{\alpha} \nu^{\alpha}[\eta]\left|\eta^{\alpha}\right|_{0}\right)^{2}+2 \epsilon \nu^{\beta}[\eta] \int_{M} \omega_{0} \rho[\eta]\left(\left|\hat{\eta}^{\beta}\right|_{0}-\left|\eta^{\beta}\right|_{0}\right)+O(\epsilon)^{2} \\
& <\int_{M} \omega_{0}\left(\sum_{\alpha} \nu^{\alpha}[\eta]\left|\eta^{\alpha}\right|_{0}\right)^{2}
\end{aligned}
$$

where in the second line we used (5.27) and (5.30) and we set $\epsilon$ to be small enough that the order $\epsilon^{2}$ term is negligible compared to the order $\epsilon$ term. Since $\nu^{\prime \alpha}=\nu^{\alpha}[\eta]$, it follows from the definition of $g_{\boldsymbol{\eta}}$ that $g_{\boldsymbol{\eta}^{\prime}}\left[\boldsymbol{\nu}^{\prime}\right]>g_{\boldsymbol{\eta}}[\nu[\boldsymbol{\eta}]]$.

For the case $\nu^{\beta}[\eta]=0$, we instead choose

$$
\begin{array}{ccc}
\eta^{\prime \alpha}=\eta^{\alpha} & \text { for } \alpha \neq \beta, & \eta^{\prime \beta}=\hat{\eta}^{\beta}, \\
\nu^{\prime \alpha}=\nu^{\alpha}[\eta] & \text { for } \alpha \neq \beta, & \nu^{\prime \beta}=\epsilon,
\end{array}
$$

where $\epsilon>0$. We have

$$
\sum_{\alpha} \nu^{\alpha \prime}\left|\eta^{\alpha \prime}\right|_{0}=\sum_{\alpha} \nu^{\alpha}[\eta]\left|\eta^{\alpha}\right|_{0}+\epsilon\left|\hat{\eta}^{\beta}\right|_{0}
$$

Expanding the definition of $g_{\eta}$ gives

$$
g_{\eta^{\prime}}\left[\nu^{\prime}\right]=g_{\eta}[\nu[\eta]]+2 \epsilon\left(\ell_{\beta}-\int_{M} \omega_{0} \rho[\eta]\left|\hat{\eta}^{\beta}\right|_{0}\right)-O(\epsilon)^{2} .
$$

Again, given (5.27), for sufficiently small $\epsilon, g_{\eta^{\prime}}\left[\nu^{\prime}\right]>g_{\eta}[\nu[\eta]]$. 


\subsubsection{From homology MAP}

The second derivation we present is the shortest one (although it contains a step that we will not fully justify). We start with the original homology minimal-area program written in terms of $\rho$ (4.22), which we reproduce here:

$$
\begin{aligned}
& \text { Minimize } \int_{M} \omega_{0} \rho^{2} \text { over } \rho \geq 0 \text { (function) } \\
& \text { subject to } \ell_{\alpha}-\int_{m} \rho|\dot{x}|_{0} \leq 0, \forall m \in C_{\alpha}, \forall \alpha \in J .
\end{aligned}
$$

We can formally combine the constraints from all the representatives of a given class $C_{\alpha}$ into one constraint:

$$
\ell_{\alpha}-\inf _{m \in C_{\alpha}} \int_{m} \rho|\dot{x}|_{0} \leq 0
$$

Notice that the second term on the left-hand side, being the infimum over a set of concave (in fact linear) functionals of $\rho$, is itself a concave functional of $\rho$, so the left-hand side is indeed a convex functional. It will be useful momentarily to have the minimization here over a convex domain, so we appeal to the equivalence of the min cut and convex min cut programs (3.48) to write (5.38) as

$$
\ell_{\alpha}-\inf _{\eta^{\alpha} \in \tilde{C}^{\alpha}} \int_{M} \omega_{0} \rho\left|\eta^{\alpha}\right|_{0} \leq 0
$$

We now have

$$
\begin{aligned}
& \text { Minimize } \int_{M} \omega_{0} \rho^{2} \text { over } \rho \text { (function) } \\
& \text { subject to } \ell_{\alpha}-\inf _{\eta^{\alpha} \in \tilde{C}^{\alpha}} \int_{M} \omega_{0} \rho\left|\eta^{\alpha}\right|_{0} \leq 0, \quad \forall \alpha \in J .
\end{aligned}
$$

We now dualize (5.40) using Lagrange multipliers $\nu^{\alpha} \geq 0$. As in the previous derivation, for sufficiently large $\rho$ the inequality constraints are strictly satisfied, so Slater's condition and therefore strong duality hold. The Lagrangian is

$$
\int_{M} \omega_{0} \rho^{2}+2 \sum_{\alpha} \nu^{\alpha}\left(\ell_{\alpha}-\inf _{\eta^{\alpha} \in \tilde{C}^{\alpha}} \int_{M} \omega_{0} \rho\left|\eta^{\alpha}\right|_{0}\right)=2 \sum_{\alpha} \nu^{\alpha} \ell_{\alpha}+\sup _{\eta \in \tilde{\mathbf{C}}} \int_{M} \omega_{0}\left(\rho^{2}-2 \rho \sum_{\alpha} \nu^{\alpha}\left|\eta^{\alpha}\right|_{0}\right)
$$

where, as in the previous derivation, $\boldsymbol{\eta}$ represents a set $\left(\eta^{\alpha}\right)_{\alpha \in J}$, and $\tilde{\mathbf{C}}$ represents the corresponding product of classes $\tilde{C}^{\alpha}$. We now minimize over $\rho$, focusing on the second term:

$$
\begin{aligned}
\inf _{\rho} \sup _{\boldsymbol{\eta} \in \tilde{\mathrm{C}}} \int_{M} \omega_{0}\left(\rho^{2}-2 \rho \sum_{\alpha} \nu^{\alpha}\left|\eta^{\alpha}\right|_{0}\right) & =\sup _{\boldsymbol{\eta} \in \tilde{\mathrm{C}}} \inf _{\rho} \int_{M} \omega_{0}\left(\rho^{2}-2 \rho \sum_{\alpha} \nu^{\alpha}\left|\eta^{\alpha}\right|_{0}\right) \\
& =\sup _{\boldsymbol{\eta} \in \tilde{\mathrm{C}}} \int_{M}-\omega_{0}\left(\sum_{\alpha} \nu^{\alpha}\left|\eta^{\alpha}\right|_{0}\right)^{2},
\end{aligned}
$$

with the minimum over $\rho$ given by (5.20). Adding the first term in the Lagrangian, $2 \sum_{\alpha} \nu^{\alpha} \ell_{\alpha}$, and maximizing over the $\nu^{\alpha}$ s gives the dual program (5.1).

In the first equality of (5.42), we switched the minimization over $\rho$ and the maximization over $\eta$. This is justified as long as the functional admits a saddle point, i.e. a configuration that is 
simultaneously a minimum with respect to $\rho$ and a maximum with respect to $\eta$; in that case, convexity with respect to $\rho$ and concavity with respect to $\eta$ guarantee that both the minimax and the maximin equal the functional's value at the saddle. We would indeed expect, but will not attempt to prove, that a saddle point exists. Alternatively, one could perhaps justify the exchange of the minimization and maximization by appealing to Sion's minimax theorem [37].

\subsubsection{From primal MAP}

We will now derive the (version 2) dual program (5.5) in a different way, by applying Lagrangian duality to the program (4.32), the primal whose dynamical variables are the scale factor $\Omega$ of the metric and a set of calibrations $u^{\alpha}$. As reviewed in subsection 3.2, this involves introducing Lagrange multipliers to enforce the constraints and then solving for the original variables, leaving a convex program expressed in terms of the Lagrange multipliers. This justifies the name "dual minimal-area program" for (5.5). We also note that one can dualize instead the program (4.33), which is written in terms of $\rho$ rather than $\Omega$. The derivation is very similar and the resulting dual program is the same.

If the optimum of the dual program is equal to the optimum of the primal we have strong duality. As reviewed earlier, a sufficient condition for strong duality is Slater's condition, which requires the existence of a feasible point at which all the inequality constraints are strictly obeyed (i.e. none are saturated). This clearly applies to the program (4.32), since closed one forms $u^{\alpha}$ with correct periods can be found and then $\Omega$ can be chosen arbitrarily large to make the inequalities strictly obeyed.

Before dualizing (4.32), we wish to write the period $\int_{m_{\alpha}} u^{\alpha}$ as an integral over the whole manifold, which we can do using the "bump form" $\eta_{m_{\alpha}}$ defined in (3.38):

$$
\int_{m_{\alpha}} u^{\alpha}=\int_{M} u^{\alpha} \wedge \eta_{m_{\alpha}}
$$

We can in fact choose an arbitrary representative $\eta_{0}^{\alpha}$ of the Poincaré(-Lefschetz) dual cohomology class $\tilde{C}^{\alpha}$, and the same result will be obtained if $u$ is closed. In terms of $\eta_{0}^{\alpha}$, the program (4.32) becomes

$$
\begin{aligned}
\text { Minimize } \int_{M} \omega_{0} \Omega \quad \text { over } & \Omega \text { (function), } u^{\alpha} \text { (one-forms) } \\
\text { subject to: } \quad\left|u^{\alpha}\right|_{0}^{2}-\Omega & \leq 0 \\
d u^{\alpha} & =0 \\
\ell_{\alpha}-\int_{M} u^{\alpha} \wedge \eta_{0}^{\alpha} & =0, \forall \alpha \in J
\end{aligned}
$$

We now introduce three Lagrange multipliers to enforce the three constraints in (5.44): a function $\lambda^{\alpha}$ required to obey $\lambda^{\alpha} \geq 0$ since it enforces the inequality constraint, and a function $\varphi^{\alpha}$ and a constant $\nu^{\alpha}$ to enforce the equality constraints:

$$
\begin{aligned}
\lambda^{\alpha} \geq 0 & \text { for }\left|u^{\alpha}\right|_{0}^{2}-\Omega \leq 0 \\
\varphi^{\alpha} & \text { for } d u^{\alpha}=0 \\
\nu^{\alpha} & \text { for } \ell_{\alpha}-\int_{M} u^{\alpha} \wedge \eta_{0}^{\alpha}=0
\end{aligned}
$$


Adding the Lagrange multiplier terms to the objective, and including some factors of 2 and signs for later convenience, we obtain the following Lagrangian functional:

$$
L=\int_{M} \omega_{0}\left[\Omega+\sum_{\alpha} \lambda^{\alpha}\left(\left|u^{\alpha}\right|_{0}^{2}-\Omega\right)\right]-2 \sum_{\alpha} \int_{M} \varphi^{\alpha} d u^{\alpha}+2 \sum_{\alpha} \nu^{\alpha}\left(\ell_{\alpha}-\int_{M} u^{\alpha} \wedge \eta_{0}^{\alpha}\right)
$$

To find the dual objective, we now minimize $L$ with respect to the variables $\Omega$ and $u^{\alpha}$ of the primal. We start by integrating by parts and reorganizing terms:

$$
\begin{aligned}
L= & 2 \sum_{\alpha} \nu^{\alpha} \ell_{\alpha}+\int_{M} \omega_{0} \Omega\left(1-\sum_{\alpha} \lambda^{\alpha}\right) \\
& +\sum_{\alpha}\left(\int_{M} \omega_{0} \lambda^{\alpha}\left|u^{\alpha}\right|_{0}^{2}-2 \int_{M} u^{\alpha} \wedge\left(\nu^{\alpha} \eta_{0}^{\alpha}+d \varphi^{\alpha}\right)-2 \int_{\partial M} \varphi^{\alpha} u^{\alpha}\right) .
\end{aligned}
$$

With the derivatives off of $u^{\alpha}$, we can now minimize the functional pointwise. All the dependence on $u^{\alpha}$ is on the second line of (5.47). Using (A.14), the terms in the bulk integrand involving $u^{\alpha}$ can be written as

$$
\lambda^{\alpha}\left\langle u^{\alpha}, u^{\alpha}\right\rangle_{0}+2\left\langle *\left(\nu^{\alpha} \eta_{0}^{\alpha}+d \varphi^{\alpha}\right), u^{\alpha}\right\rangle_{0}
$$

where $*$ is the Hodge star with respect to any metric in the Weyl class of $g^{0}$. This has a minimum with respect to $u^{\alpha}$ as it is the sum of a quadratic function of $u^{\alpha}$ with positive coefficient and a linear function of $u^{\alpha}$. At the minimum,

$$
u^{\alpha}=-\frac{1}{\lambda^{\alpha}} *\left(\nu^{\alpha} \eta_{0}^{\alpha}+d \varphi^{\alpha}\right)
$$

The boundary integrand in (5.47) is $\varphi^{\alpha} u^{\alpha}$; this has a minimum only if

$$
\left.\varphi^{\alpha}\right|_{\partial M}=0,
$$

in which case it vanishes. This boundary condition is a constraint on the dual variable $\varphi^{\alpha}$. With these results, the Lagrangian is now

$$
\inf _{u^{\alpha}} L=2 \sum_{\alpha} \nu^{\alpha} \ell_{\alpha}+\int_{M} \omega_{0} \Omega\left(1-\sum_{\alpha} \lambda^{\alpha}\right)-\int_{M} \omega_{0} \sum_{\alpha} \frac{1}{\lambda^{\alpha}}\left|\nu^{\alpha} \eta_{0}^{\alpha}+d \varphi^{\alpha}\right|_{0}^{2}
$$

We still have to minimize with respect to $\Omega$, which appears linearly in $L$. If we think of $\Omega$ as an unconstrained variable, the answer is clear: if the factor multiplying $\Omega$ is non-zero the minimum does not exist. So the existence of a minimum requires that this factor vanishes, leaving $\Omega$ undetermined:

$$
\sum_{\alpha} \lambda^{\alpha}=1
$$

Using this we finally obtain the dual objective:

$$
2 \sum_{\alpha} \nu^{\alpha} \ell_{\alpha}-\int_{M} \omega_{0} \sum_{\alpha} \frac{1}{\lambda^{\alpha}}\left|\nu^{\alpha} \eta_{0}^{\alpha}+d \varphi^{\alpha}\right|_{0}^{2}
$$


The dual program is therefore

$$
\begin{aligned}
& \text { Maximize }\left[2 \sum_{\alpha} \nu^{\alpha} \ell_{\alpha}-\int_{M} \omega_{0} \sum_{\alpha} \frac{1}{\lambda^{\alpha}}\left|\nu^{\alpha} \eta_{0}^{\alpha}+d \varphi^{\alpha}\right|_{0}^{2}\right] \\
& \text { over } \lambda^{\alpha}, \varphi^{\alpha} \text { (functions), } \nu^{\alpha} \text { (constants) } \\
& \text { subject to: } \quad \lambda^{\alpha} \geq 0, \\
& -1+\sum_{\alpha} \lambda^{\alpha}=0, \\
& \qquad\left.\varphi^{\alpha}\right|_{\partial M}=0, \quad \forall \alpha \in J .
\end{aligned}
$$

Since there are no derivatives acting on $\lambda^{\alpha}$, we might as well go ahead and solve for it. To maximize over $\lambda^{\alpha} \geq 0$, we only need to consider the second term in the objective since $\lambda^{\alpha}$ does not appear in the first. Since the second term is the sum of non-positive terms we must minimize the second term with its sign flipped. Since the integrand is manifestly positive, this amounts to solving, pointwise and for fixed $\alpha$, the following mini-program:

$$
\begin{gathered}
\text { Minimize } \sum_{\alpha} \frac{c_{\alpha}^{2}}{\lambda^{\alpha}} \text { over } \lambda^{\alpha} \\
\text { subject to: } \quad \lambda^{\alpha} \geq 0, \\
-1+\sum_{\alpha} \lambda^{\alpha}=0 .
\end{gathered}
$$

Here the $c_{\alpha}$ 's are defined by

$$
c_{\alpha}:=\left|\nu^{\alpha} \eta_{0}^{\alpha}+d \varphi^{\alpha}\right|_{0}
$$

The $c_{\alpha}$ 's and the $\lambda^{\alpha}$ 's are functions on the surface, but for the mini-program which is defined pointwise, they are just some non-negative constants. We can quickly solve this program but a slightly more general version is useful:

$$
\begin{gathered}
\text { Minimize } \sum_{\alpha} \frac{c_{\alpha}^{2}}{\lambda^{\alpha}} \text { over } \lambda^{\alpha} \\
\text { subject to: } \quad \lambda^{\alpha} \geq 0, \\
-1+\sum_{\alpha} \lambda^{\alpha} \leq 0 .
\end{gathered}
$$

In here we replaced the equality setting the sum of $\lambda^{\alpha}$ 's equal to one by an inequality. In fact we will show that at the minimum the equality holds. This modified program would arise had we treated $\Omega$ as a variable satisfying the implicit constraint $\Omega \geq 0$. In this case the earlier minimization over $\Omega$ in (5.47) is only possible when the coefficient multiplying $\Omega$ is positive or zero, i.e.

$$
\sum_{\alpha} \lambda^{\alpha} \leq 1
$$

Note that if this inequality is not saturated then we must have $\Omega=0$ at the minimum. This indeed suggests that the inequality must be saturated. It is now a short exercise to show that the minimum of the objective occurs for

$$
\lambda^{\alpha *}=\frac{c_{\alpha}}{\sum_{\beta} c_{\beta}}
$$


For this value of $\lambda$ the objective is

$$
\sum_{\alpha} \frac{c_{\alpha}^{2}}{\lambda^{\alpha *}}=\left(\sum_{\alpha} c_{\alpha}\right)^{2}=\left(\sum_{\alpha}\left|\nu^{\alpha} \eta_{0}^{\alpha}+d \varphi^{\alpha}\right|_{0}\right)^{2} .
$$

Substituting this optimum back into the program (5.54) gives (5.5).

\section{Simple examples}

In order to gain some intuition for the primal and dual programs, in this section we apply them to two exactly solvable cases where the minimal-area metric is known, namely the cylinder (or annulus) and the torus.

\subsection{Cylinder}

An annulus, or finite cylinder or ring domain, $R$ is a planar connected Riemann surface with two homotopic disjoint boundary components, each of which is a circle. A natural minimal-area problem asks for the conformal metric of least area such that all curves homotopic to the boundaries are longer than or equal to $\ell_{s}$. The solution of this problem is well-known and will be briefly explained below. We will also solve the problem using the primal program as well as using the dual program.
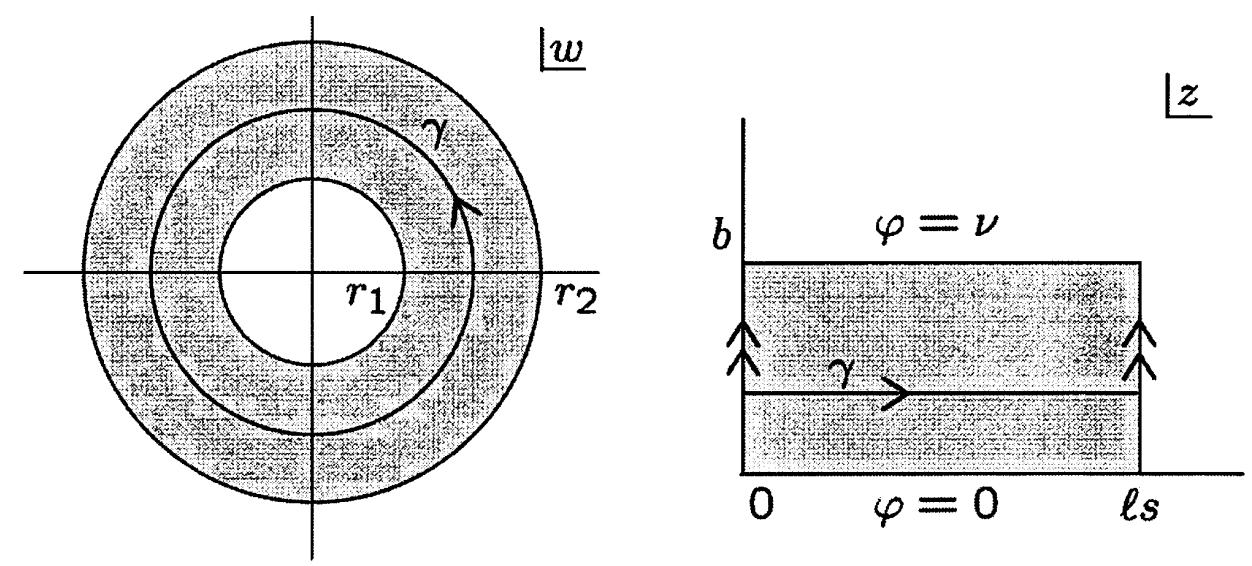

Figure 7: An annulus $r_{1} \leq|w| \leq r_{2}$, with a core curve $\gamma$. This annulus is mapped to a rectangle in the $z$ plane, with its vertical edges identified. The map $z=\frac{\ell_{s}}{2 \pi i} \ln \frac{w}{r_{2}}$.

It is a familiar result of conformal mappings that any annulus can be presented as a canonical annulus $r_{1} \leq|w| \leq r_{2}$ on the complex $w$ plane, for some fixed value of the ratio $r_{2} / r_{1}$, as shown in Figure 7. This presentation is unique up to a constant scaling of $w$. The modulus $M$ of the annulus is defined by

$$
M \equiv \frac{1}{2 \pi} \ln \frac{r_{2}}{r_{1}} .
$$

The $w$-plane annulus can be mapped to a $z$-plane rectangle with its vertical edges identified:

$$
z=\frac{\ell_{s}}{2 \pi i} \ln \frac{w}{r_{2}} .
$$


With $z=x+i y$, the rectangle extends over $x \in\left[0, \ell_{s}\right]$ and $y \in[0, b]$, where $b$ is

$$
b=\frac{\ell_{s}}{2 \pi} \ln \frac{r_{2}}{r_{1}} .
$$

$b$ is the height of the cylinder form by the identification of the vertical edges of the rectangle. In the rectangle picture the modulus $M$ is defined as the ratio of the height $b$ and the circumference $\ell_{s}$ :

$$
M=\frac{b}{\ell_{s}},
$$

which on account of the expression for $b$ is manifestly equal to $M$ as given in (6.1). It is simplest to think of the annulus as the identified rectangular region in the $z$ plane and we will do that in the following.

The minimal area problem would then state that all closed curves beginning on the left vertical edge and ending on the right vertical edge should be longer than or equal to $\ell_{s}$. Those are the curves homologous to the core curve $\gamma$ of the annulus, shown in Figure 7. Let $A$ be the minimal area metric. The metric $d s=\rho|d z|$ with $\rho=1$ is admissible and has area $\ell_{s} b$. Therefore,

$$
A \leq \ell_{s} b \text {. }
$$

On the other hand, the constraint that any closed curve with fixed $y$ be long enough is

$$
\int_{0}^{\ell_{s}} d x \rho(x, y) \geq \ell_{s}
$$

Integrating this equation over $y$ to get a full two-dimensional integral over the annulus $R$,

$$
\int_{R} d x d y \rho \geq \ell_{s} b
$$

By Schwarz's inequality with $\rho=\rho \cdot 1$, the left-hand side above, which is manifestly positive, satisfies

$$
\int_{R} d x d y \rho \leq \sqrt{\int_{R} d x d y \rho^{2} \int_{R} d x d y}=\sqrt{A(\rho) \ell_{s} b},
$$

where $A(\rho)$ is the area of the metric $\rho$. Squaring this relation we get

$$
A(\rho) \geq \frac{1}{\ell_{s} b}\left(\int_{R} d x d y \rho\right)^{2} \geq \ell_{s} b
$$

making use of (6.7). This implies that

$$
A \geq \ell_{s} b .
$$

Together with (6.5) this proves that $\rho=1$ is the extremal metric and the extremal area is $\ell_{s} b$ :

$$
A=\ell_{s} b=\ell_{s}^{2} M=\frac{b^{2}}{M} .
$$

Let us now consider the solution via the primal program in its version 2 (4.39). We have a single homology $C$ represented by closed curves, like $\gamma$, stretching from the left to the right boundaries of the identified rectangle. Let us call the corresponding calibration $u$. This calibration takes the form

$$
u=d x+d \phi .
$$


Here the piece $d x$ is required for the calibration to have the right period: $\int_{C} u=\int_{C} d x=\ell_{s}$. The function $\phi$ on the surface generates the trivial part of the calibration. We do not include a $d y$ component to the calibration because the surface is symmetric under reflections about a horizontal line $y=b / 2$ and $d y$ would not be invariant under such reflection while $u$ must be because it contains $d x$. The constraint $\Omega \geq|u|_{0}^{2}$ with fiducial metric $\rho_{0}=1$ gives

$$
\Omega \geq\left(1+\partial_{x} \phi\right)^{2}+\left(\partial_{y} \phi\right)^{2} .
$$

We can impose the symmetry $(x, y) \rightarrow(x+c, y)$ for any real constant $c$, this is the rotational symmetry of the annulus. Applied to our calibration this requires that $\phi$ be constant along $x$. The above condition then gives

$$
\Omega \geq 1+\left(\partial_{y} \phi\right)^{2}
$$

Since we are trying to minimize $\Omega$ pointwise, we find $\partial_{y} \phi=0$ and conclude that $\Omega=1$. The minimum of the primal is therefore $\int_{R} \Omega d x d y=\int_{R} d x d y=\ell_{s} b$, as found before.

Now consider the maximization of the dual objective in (5.7), called here $\mathcal{F}$, applied to the annulus $R$ :

$$
\mathcal{F}=2 \nu \ell_{s}-\int_{R} \omega_{0}|d \varphi|_{0}^{2}
$$

Here we take the fiducial metric to be the constant unit metric on the identified rectangle in the $z$ plane, so $\omega_{0}=d x d y$. We want to show that maximizing $\mathcal{F}$ over $\varphi$ and $\nu$ leads to an optimum where $\mathcal{F}$ equals the previously determined minimal area.

We fix the value $\varphi=0$ at the outer radius of the annulus, namely the horizontal segment $x \in\left[0, \ell_{s}\right], y=0$ (see Figure 7). This is sensible as the dual requires the value of $\varphi$ at a boundary to be zero. The value $\varphi=\nu$ is fixed at the inner circle of the annulus or the horizontal segment $x \in$ $\left[0, \ell_{s}\right], y=b$. As explained before (see (5.9)), this takes into account the discontinuity requirement on $\varphi$. Considering the rotational symmetry of the boundary conditions and fiducial metric, we could impose a rotational symmetry on $\varphi$; however, it turns out to be just as easy to solve for $\varphi$ without imposing this symmetry. Consider now the variation of $\varphi$ with these boundary conditions, taking $\nu$ to be fixed. We then have

$$
\begin{aligned}
\delta \mathcal{F} & =-\delta \int_{R} d^{2} x \nabla \varphi \cdot \nabla \varphi=-2 \int_{R} d^{2} x \nabla \delta \varphi \cdot \nabla \varphi \\
& =-2 \int_{\partial R} \delta \varphi \nabla \varphi \cdot \hat{n}+2 \int_{R} \delta \varphi \nabla^{2} \varphi .
\end{aligned}
$$

Since $\delta \varphi$ vanishes at the boundary, the equation that fixes $\varphi$ is $\nabla^{2} \varphi=0$. The solution satisfying the boundary conditions is thus unique and takes the form

$$
\varphi=\nu \frac{y}{b} \rightarrow \nabla \varphi=\frac{\nu}{b} \hat{y} \quad \rightarrow \quad|\nabla \varphi|^{2}=\frac{\nu^{2}}{b^{2}}=\frac{\nu^{2}}{\ell_{s}^{2} M^{2}}
$$

recalling that $b=M \ell_{s}$. We now evaluate the objective, finding

$$
\mathcal{F}=2 \nu \ell_{s}-\frac{\nu^{2}}{\ell_{s}^{2} M^{2}} \int_{R} d x d y=2 \nu \ell_{s}-\frac{\nu^{2}}{\ell_{s}^{2} M^{2}} \ell_{s} b=2 \nu \ell_{s}-\frac{\nu^{2}}{M}
$$

We must now maximize over $\nu$. The critical point is $\nu=\ell_{s} M$ and this gives, as desired

$$
\mathcal{F}=\ell_{s}^{2} M=A .
$$




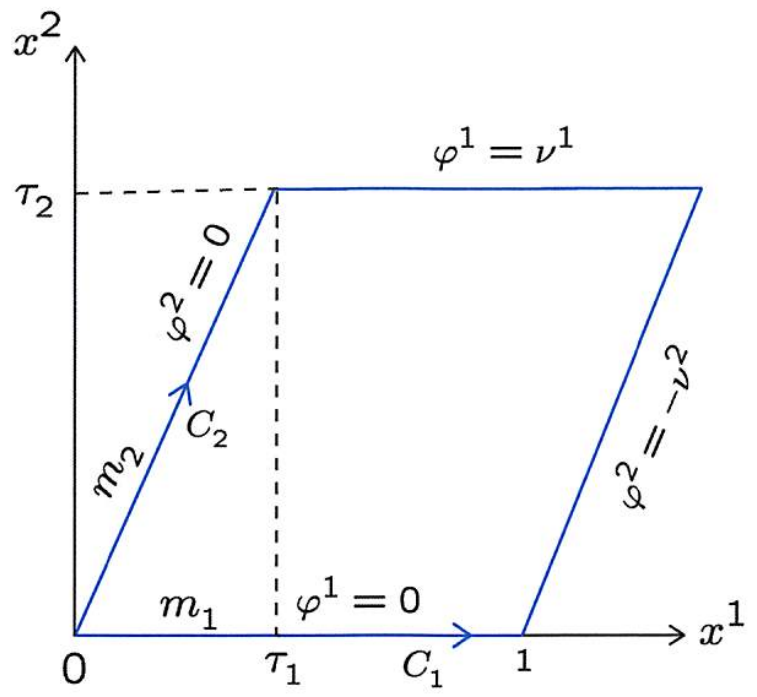

Figure 8: Torus with modular parameter $\tau=\tau_{1}+i \tau_{2}$. Indicated are the homology cycle $C_{1}$, along the bottom side, and $C_{2}$ along the left side the parallelogram. In the presentation (6.26) of the dual configuration, the function $\varphi^{1}$ is discontinuous on the representative $m_{1}$ (bottom or top side) and the function $\varphi^{2}$ is discontinuous on the representative $m_{2}$ (left or right side).

\subsection{Torus}

We now consider the torus with length constraints on two intersecting cycles. We present the torus in the usual form, with modular parameter $\tau=\tau_{1}+i \tau_{2}$, coordinates $x^{1}, x^{2}$ with periodicities $\left(x^{1}, x^{2}\right) \sim\left(x^{1}+1, x^{2}\right) \sim\left(x^{1}+\tau_{1}, x_{2}+\tau_{2}\right)$, and constant fiducial metric $g_{\mu \nu}^{0}=\delta_{\mu \nu}$. The torus is shown in Figure 8. Letting the cycles $C_{1}$ and $C_{2}$ be the bottom and left sides of the parallelogramshaped fundamental domain of the torus, we require that all representatives of these two cycles have length at least 1 . Note that this is not a modular-invariant condition, and does not imply that representatives of all non-trivial cycles have length at least 1.

We start with the original form (4.15) of the minimal-area problem. The torus with the fiducial metric has a 2-parameter family of continuous isometries. As explained in subsection 3.1, we can impose this symmetry on the configuration, in other words we can set the Weyl factor $\Omega$ to be constant. This also follows from the fact that the minimal-area metric $\Omega^{*} g_{\mu \nu}^{0}$ is unique, as explained in section 4.3 .

The closed geodesics in the class $C_{1}$ are the curves of constant $x^{2}$, which have length $\sqrt{\Omega}$. The ones in the class $C_{2}$ are the curves of constant $\tau_{1} x^{2}-\tau_{2} x^{1}$, which have length $\sqrt{\Omega\left(\tau_{1}^{2}+\tau_{2}^{2}\right)}$. The length conditions are therefore:

$$
\Omega \geq 1, \quad \text { and } \quad \Omega \geq \frac{1}{\tau_{1}^{2}+\tau_{2}^{2}} .
$$

The coordinate area is $\tau_{2}$, so the proper area is $\tau_{2} \Omega$. The minimal area $A_{*}$ arises from the minimum possible $\Omega$ satisfying the above constraints. We therefore have

$$
A_{*}=\tau_{2} \max \left\{1, \frac{1}{\tau_{1}^{2}+\tau_{2}^{2}}\right\} \text {. }
$$

If the first argument of the max function is the largest, the curves saturating the length condition 
are in the class $C_{1}$; if the second argument of the max function is the largest, they are in the class $C_{2}$.

We now consider the program (4.39) where we work with calibrations $u^{1}$ and $u^{2}$ written in terms of basis one-forms. Again, we can assume that these are invariant under the continuous isometries of the fiducial metric on the torus. A basis of closed one-forms is given by $d x^{1}$ and $d x^{2}$, which have the following periods:

$$
\int_{C_{1}} d x^{1}=1, \quad \int_{C_{1}} d x^{2}=0, \quad \int_{C_{2}} d x^{1}=\tau_{1}, \quad \int_{C_{2}} d x^{2}=\tau_{2} .
$$

We now write $u^{1}$ and $u^{2}$ as general linear combinations of $d x^{1}$ and $d x^{2}$ with unknown coefficients; invariance under the torus isometries implies the scalars $\phi^{1}$ and $\phi^{2}$ are constant and can be dropped. Applying the constraints $\int_{C_{1}} u^{1}=\int_{C_{2}} u^{2}=1$, we find

$$
u^{1}=d x^{1}+c_{1} d x^{2}, \quad u^{2}=\frac{1}{\tau_{2}} d x^{2}+c_{2}\left(d x^{1}-\frac{\tau_{1}}{\tau_{2}} d x^{2}\right),
$$

where $c_{1}$ and $c_{2}$ are to be determined. We then have

$$
\Omega \geq\left|u^{1}\right|_{0}^{2}=1+c_{1}^{2}, \quad \Omega \geq\left|u^{2}\right|_{0}^{2}=c_{2}^{2}+\frac{\left(1-c_{2} \tau_{1}\right)^{2}}{\tau_{2}^{2}} \bigcirc
$$

The minimum of $\left|u^{1}\right|_{0}^{2}$ over $c_{1}$ is clearly 1 , while the minimum of $\left|u^{2}\right|_{0}^{2}$ over $c_{2}$ is easily computed to equal $1 /\left(\tau_{1}^{2}+\tau_{2}^{2}\right)$. The minimum allowed value of $\Omega$ is the larger of these two, so the result agrees with (6.21). The tangent to the saturating curves is along the vector $\hat{u}$ of the calibration that saturates the inequality with the scale factor.

Finally, we solve the dual program. In the form (5.7), the program requires choosing representatives $m_{\alpha}$, which necessarily break the torus's continuous isometries. Therefore we will work instead with the dual in the form (5.1), where the variables are the one-forms $\eta^{\alpha}$. The requirement $\eta^{\alpha} \in \tilde{C}^{\alpha}$, which implies $\int_{M} u \wedge \eta^{\alpha}=\int_{C_{\alpha}} u$ for any closed one-form $u$, together with invariance under the isometries, completely fixes the $\eta^{\alpha}$ :

$$
\eta^{1}=\frac{1}{\tau_{2}} d x^{2}, \quad \eta^{2}=-d x^{1}+\frac{\tau_{1}}{\tau_{2}} d x^{2} .
$$

Having obtained the $\eta^{\alpha}$, we can write them in the form of the program (5.7). That is, we can write $\nu^{\alpha} \eta^{\alpha}=\nu^{\alpha} \eta_{m_{\alpha}}+d \varphi^{\alpha}$ for some representatives $m_{\alpha}$ and functions $\varphi^{\alpha}$. Choosing $m_{1}$ along the bottom (or top) edge of the parallelogram and $m_{2}$ along the left (or right) edge, the required functions $\varphi^{\alpha}$ are

$$
\varphi^{1}=\nu^{1} \frac{x^{2}}{\tau_{2}}, \quad \varphi^{2}=\nu^{2}\left(-x^{1}+\frac{\tau_{1}}{\tau_{2}} x^{2}\right) .
$$

These are functions on the fundamental domain of the torus, shown in Figure 8. On the full torus, they are discontinuous.

We now have

$$
\nu^{1}\left|\eta^{1}\right|_{0}=\frac{\nu^{1}}{\tau_{2}}, \quad \nu^{2}\left|\eta^{2}\right|_{0}=\frac{\nu^{2}}{\tau_{2}} \sqrt{\tau_{1}^{2}+\tau_{2}^{2}},
$$

and, in either form of the dual program, the following value for the objective:

$$
\text { Objective }=2\left(\nu^{1}+\nu^{2}\right)-\frac{1}{\tau_{2}}\left(\nu^{1}+\nu^{2} \sqrt{\tau_{1}^{2}+\tau_{2}^{2}}\right)^{2} .
$$

We now need to maximize over $\nu^{1}$ and $\nu^{2}$. There are three possible cases for the maximum: 

1. $\nu^{1}>0$ and $\nu^{2}>0$,
2. $\nu^{1}>0$ and $\nu^{2}=0$,
3. $\nu^{1}=0$ and $\nu^{2}>0$.

The first case is ruled out by calculating the gradient of the objective and showing that it doesn't vanish anywhere (except if $\tau_{1}^{2}+\tau_{2}^{2}=1$, in which case there is a line of critical points at $\nu^{1}+\nu^{2}=\tau_{2}$ ). The maximum on the $\nu^{1}>0, \nu^{2}=0$ half-line is at $\nu^{1}=\tau_{2}$ and has value $\tau_{2}$. The maximum on the $\nu^{1}=0, \nu^{2}>0$ half-line is at $\nu^{2}=\tau_{2} /\left(\tau_{1}^{2}+\tau_{2}^{2}\right)$ and has value $\tau_{2} /\left(\tau_{1}^{2}+\tau_{2}^{2}\right)$. The maximum of (6.28) thus agrees with (6.21), consistent with strong duality. As we will see in subsection 7.1 , in the presentation (6.26) the level sets for $\varphi^{\alpha}$ with non-zero $\nu^{\alpha}$ are saturating geodesics in the class $C_{\alpha}$.

\section{Properties of the minimal-area metric}

We now have two convex programs for the (homological) minimal-area problem: a primal (4.32), in which the variables are the Weyl factor $\Omega$ of the metric and a set of one-forms $u^{\alpha}$, and a dual (5.1) or (5.7) where the variables are a set of one-forms $\eta^{\alpha}$ or functions $\varphi^{\alpha}$ and a set of constants $\nu^{\alpha}$. In this section, we will explore properties of the solutions of these programs, their relation to each other, and what they tell us about the geometry of the minimal-area metric. In subsection 7.1, we explain how to extract the saturating geodesics in each homology class from primal and dual solutions. In subsection 7.2, we derive simple formulas relating the dual solution to the primal solution and to its total area, and in subsection 7.3 we use them to study properties of bands of geodesics. In subsection 7.4, we get more intuition for the dual by understanding how it implements non-trivial properties that we know are obeyed by the primal solution. Finally, in 7.5, we explain how the dual program can be understood as a generalization of a formula by Jenkins and Strebel for the minimal-area metric for the homotopy MAP problem (4.14) where the constrained homotopy classes are represented by non-intersecting curves.

In subsections 7.1-7.3 we consider only optimal field configurations, so to avoid cluttering the notation we drop the superscript $*$; thus $\Omega$ means $\Omega^{*}$, etc.

\subsection{Saturating geodesics}

Consider a representative of $C_{\alpha}$ that has length $\ell_{\alpha}$ and therefore saturates the length condition. Such a curve must be locally length-minimizing, else there exist representatives that violate the length condition. Except where it touches a singularity or boundary of $M$, the curve must therefore be a closed geodesic (or a collection of closed geodesics, if the curve has multiple components). With a slight abuse of language we will call a $C_{\alpha}$ representative of length $\ell_{\alpha}$ an $\alpha$-geodesic. In the original minimal-area problem, in which $\ell_{\alpha}=\ell_{s}$ for all $\alpha$, all $\alpha$-geodesics are in fact systolic geodesics.

As we will now show, the location of the $\alpha$-geodesics can be readily extracted from any solution to either the primal or the dual program.

We begin with the primal. An $\alpha$-geodesic is calibrated, in the metric $\Omega g^{0}$, by the calibration form $u^{\alpha}$ : the constraint $\left|u^{\alpha}\right|_{0}^{2} \leq \Omega$ is saturated on that curve and furthermore the vector $\hat{u}^{\alpha}$ is tangent to the curve (see (4.29)). The converse is also true: Any closed integral curve of $\hat{u}^{\alpha}$ on 
which $\left|u^{\alpha}\right|_{0}^{2}=\Omega$ everywhere is an $\alpha$-geodesic. This allows one to easily identify the $\alpha$-geodesics from a solution to the primal program (4.32).

The $\alpha$-geodesics are the closed integral curves of $u^{\alpha}$ on which $\left|u^{\alpha}\right|_{0}^{2}=\Omega$ everywhere.

We should point out that it is possible to have $\left|u^{\alpha}\right|_{0}^{2}=\Omega$ at a point that is not on an $\alpha$-geodesic. To confirm the existence of an $\alpha$ geodesic, the full integral curve of $u^{\alpha}$ must be examined.

The $\alpha$-geodesics can also easily be extracted from the solution to the dual program, in any of the forms given in subsection 5.1. Specifically, we will show below that, given a solution $\left(\nu^{\alpha}, \eta^{\alpha}\right)$ to (5.1), if $\nu^{\alpha}>0$ for a given $\alpha$ then the level sets $m_{\eta^{\alpha}}(t)(t \in \mathbb{R} / \mathbb{Z})$ defined in (3.44) are $\alpha$-geodesics. By construction, these are the curves orthogonal to $\eta^{\alpha}$ in the region of $M$ where $\eta^{\alpha} \neq 0$. We can also write this in terms of the variable $\varphi^{\alpha}$ appearing in (5.7). Away from the fiducial representative $m_{\alpha}$ on which $\varphi^{\alpha}$ jumps, $\eta^{\alpha}=d \varphi^{\alpha} / \nu^{\alpha}$, so the level sets $m_{\eta^{\alpha}}(t)$ are the curves of constant $\varphi^{\alpha}$ in the region where $d \varphi^{\alpha} \neq 0$ :

Wherever $d \varphi^{\alpha} \neq 0$, the curves of constant $\varphi^{\alpha}$ are $\alpha$-geodesics.

The converse to this statement does not quite always hold; it is possible for $d \varphi^{\alpha}$ to vanish at a point through which an $\alpha$-geodesic passes. ${ }^{9}$ However, we expect the converse to hold generically.

The statement that the level sets $m_{\eta^{\alpha}}(t)$ are $\alpha$-geodesics can be deduced from any of the three derivations of the dual program in subsection 5.2. For concreteness consider the derivation in subsection 5.2.1. Let $\left(\nu^{\alpha}, \eta^{\alpha}\right)$ be a solution to the dual MAP (5.1). Apply complementary slackness to the duality relating (5.18) and (5.21). If, for some $\alpha, \nu^{\alpha}>0$, then the corresponding constraint in (5.18) is saturated, i.e.

$$
\int_{M} \omega_{0} \rho\left|\eta^{\alpha}\right|_{0}=\ell_{\alpha}
$$

where $\rho$ is given by $(5.20)$ :

$$
\rho=\sum_{\alpha} \nu^{\alpha}\left|\eta^{\alpha}\right|_{0}
$$

As argued in the derivation, this is the solution to the homology MAP (4.22). The left-hand side of (7.3) equals $\int_{M} \omega\left|\eta^{\alpha}\right|$, which by the coarea formula (3.45) is the average length of the level sets $m_{\eta^{\alpha}}(t)$, so

$$
\int_{0}^{1} d t \text { length }\left(m_{\eta^{\alpha}}(t)\right)=\ell_{\alpha}
$$

Since $\rho$ is feasible, every representative of $C_{\alpha}$ has length at least $\ell_{\alpha}$. The only way the average can equal $\ell_{\alpha}$ is then if every level set has length equal to $\ell_{\alpha}$. So the level sets are indeed $\alpha$-geodesics.

In the language of the Dual MAP v3, for a solution $\left(\nu^{\alpha}, \varphi^{\alpha}\right)$ we have:

$$
\rho=\sum_{\alpha}\left|d \varphi^{\alpha}\right|_{0}
$$

\footnotetext{
${ }^{9}$ For example, if the $\alpha$-geodesics do not foliate the entire surface, then the boundary of the region they foliate is itself an $\alpha$-geodesic. But if $d \varphi^{\alpha}$ is continuous, then it vanishes on that boundary. This situation will be seen on the Swiss-cross/torus-with-a-boundary surface studied in [22]. A more extreme example is provided by the square torus $\tau_{1}=0, \tau_{2}=1$, using the analysis of the dual problem in section 6.2. In this case the dual objective is maximized for $\nu^{1}+\nu^{2}=\tau_{2}=1$. We can choose to take $\nu^{1}=1$ and $\nu^{2}=0$, in which case $\varphi^{2}$ is constant and $d \varphi^{2}=0$. Despite this vanishing gradient, the torus has vertical 2-geodesics, in addition to the horizontal 1-geodesics.
} 


\subsection{The metric and the primal and dual solutions}

In the previous subsection, we noted that the solutions to the primal and dual MAPs tell us both the minimal-area metric and the location of the saturating geodesics. Here we will explore further these solutions and their relationship to each other.

Denote by $A$ the area of the minimal-area metric, which is the optimal value of the homology, primal, and dual MAPs. Given the solution $\rho$ to the homology MAP and $\left(\nu^{\alpha}, \eta^{\alpha}\right)$ to the dual MAP, we have

$$
\int_{M} \omega_{0} \rho^{2}=A=2 \sum_{\alpha} \nu^{\alpha} \ell_{\alpha}-\int_{M} \omega_{0}\left(\sum_{\alpha} \nu^{\alpha}\left|\eta^{\alpha}\right|_{0}\right)^{2} .
$$

However, from (7.4), the second term on the right-hand side is minus the left-hand side. This implies the following simple relation between the $\nu^{\alpha}$ s and the area:

$$
A=\sum_{\alpha} \nu^{\alpha} \ell_{\alpha}, \longrightarrow
$$

This result gives an intuitive interpretation for the value of the parameters $\nu^{\alpha}$ in the solution. The total area for the extremal metric equals the sum of areas of flat rectangles of height $\nu^{\alpha}$ and length $\ell_{\alpha}$. We get a contribution from each band of $\alpha$-geodesics. While these bands can cross and the extremal metric is neither flat nor simple, the area is indeed given by a simple formula, as if the surface were built with flat rectangles. We will rederive the result (7.8) from a local point of view in the next subsection.

The relation (7.4) is very useful. Dividing both sides by $\rho$, and noting that $|\cdot|=\rho^{-1}|\cdot|_{0}$, yields a simple "sum rule":

$$
\sum_{\alpha} \nu^{\alpha}\left|\eta^{\alpha}\right|=1
$$

In terms of the variables of $(5.7)$,

$$
\eta^{\alpha}=\eta_{m_{\alpha}}^{\alpha}+\frac{1}{\nu^{\alpha}} d \varphi^{\alpha}
$$

with $m_{\alpha}$ the fiducial representative of $C_{\alpha}$ on which $\varphi^{\alpha}$ is required to jump by $-\nu^{\alpha}$. Away from the $m_{\alpha}$ 's, (7.9) thus becomes

$$
\sum_{\alpha}\left|d \varphi^{\alpha}\right|=1 \quad \text { at every point on } M
$$

This "sum rule" implies that at every point $d \varphi^{\alpha}$ must be non-zero for at least one $\alpha$, recovering the fact that at least one saturating curve passes through every point in $M$. For a given $\alpha$, the $\alpha$-geodesics foliate the region through which they pass. The fact that a region is foliated by such curves does not itself constrain the geometry, but (7.11) provides extra information.

Finally, we use the derivation of the dual MAP in subsection 5.2.3 to relate the solutions of the primal and dual MAPs. Recall that (3.16) says that the primal solution minimizes the Lagrangian function with the dual variables set to the dual solution. Using this fact, and combining (5.49), (5.56), and (5.59), we find:

$$
u^{\alpha}=-\frac{*\left(\nu^{\alpha} \eta_{0}^{\alpha}+d \varphi^{\alpha}\right)}{\left|\nu^{\alpha} \eta_{0}^{\alpha}+d \varphi^{\alpha}\right|_{0}} \sum_{\beta}\left|\nu^{\beta} \eta_{0}^{\beta}+d \varphi^{\beta}\right|_{0}
$$


Using (7.4), we recognize the sum on the right-hand side as $\rho$, which we can use to replace the fiducial norm $|\cdot|_{0}$ in the denominator by $|\cdot|$ :

$$
u^{\alpha}=-\frac{*\left(\nu^{\alpha} \eta_{0}^{\alpha}+d \varphi^{\alpha}\right)}{\left|\nu^{\alpha} \eta_{0}^{\alpha}+d \varphi^{\alpha}\right|}
$$

If we choose the fiducial one-form $\eta_{0}^{\alpha}$ to be the bump form $\eta_{m_{\alpha}}$, then away from $m_{\alpha}$ we have simply

$$
u^{\alpha}=-\frac{* d \varphi^{\alpha}}{\left|d \varphi^{\alpha}\right|}
$$

This is consistent with (7.1) and (7.2). Equation (7.14) does not determine $u^{\alpha}$ where $d \varphi^{\alpha}=0$, that is, away from the $\alpha$-geodesics. We might have anticipated this since $u^{\alpha}$ is highly underdetermined away from these curves. The condition $d u^{\alpha}=0$ is in fact the Euler-Lagrange equation for $\varphi^{\alpha}$ following from the dual objective in (5.7).

\subsection{Normal coordinates for bands of geodesics}

Fix a value of $\alpha$. As discussed in section 7.1, $\varphi^{\alpha}$ is constant along any $\alpha$-geodesic and (generically) has non-zero gradient normal to it. We can therefore use $\varphi^{\alpha}$ as a local coordinate for the region foliated by a band of $\alpha$-geodesics. In fact, we can construct a Gaussian normal coordinate system using such a band. Consider the tangent vector $\hat{u}^{\alpha}$ to the geodesics and the orthogonal vector field $\widehat{d \varphi^{\alpha}}$. Now use the orthogonal vector field to build integral curves orthogonal to the geodesics. By Gauss's lemma the distance along the geodesics between two orthogonal integral curves is a constant. ${ }^{10}$ Thus there is a function $x^{\alpha}$ that provides a parameterization of the $\alpha$-geodesics by length and is constant along the orthogonal integral curves. The function $x^{\alpha}$ will be our first coordinate. The calibration $u^{\alpha}$ can be identified locally with the exterior derivative of the function $x^{\alpha}$ :

$$
u^{\alpha}=d x^{\alpha}
$$

Since the period of $u^{\alpha}$ is $\ell_{\alpha}, x^{\alpha}$ ranges from 0 to $\ell_{\alpha}$. The second coordinate is $\varphi^{\alpha}$ which, as required, is constant along the systolic geodesics (see Figure 9). The form of the cotangent space metric $g^{-1}$ is constrained by the conditions $\left|d x^{\alpha}\right|=1$ (since $\left|u^{\alpha}\right|=1$ ) and the orthogonality $\left\langle d x^{\alpha}, d \varphi^{\alpha}\right\rangle=0$. This determines $g^{-1}$ up to one unknown function $h_{\alpha}$ :

$$
g^{-1}=\left(\begin{array}{cc}
1 & 0 \\
0 & h_{\alpha}^{2}\left(x^{\alpha}, \varphi^{\alpha}\right)
\end{array}\right) \text {. }
$$

The interpretation of $h_{\alpha}$ arises from the length condition

$$
\left|d \varphi^{\alpha}\right|=\left|h_{\alpha}\right| .
$$

Given the above form of $g^{-1}$ the metric $g$ follows immediately and gives

$$
d s^{2}=\left(d x^{\alpha}\right)^{2}+\frac{1}{h_{\alpha}^{2}}\left(d \varphi^{\alpha}\right)^{2}
$$

\footnotetext{
${ }^{10}$ As explained in [34] p.137: If segments of equal length are laid off along geodesics orthogonal to a univalent curve, their endpoints determine an orthogonal trajectory to the family of geodesics. For our case, the univalent curve is any integral curve of $\widehat{d \varphi}^{\alpha}$.
} 


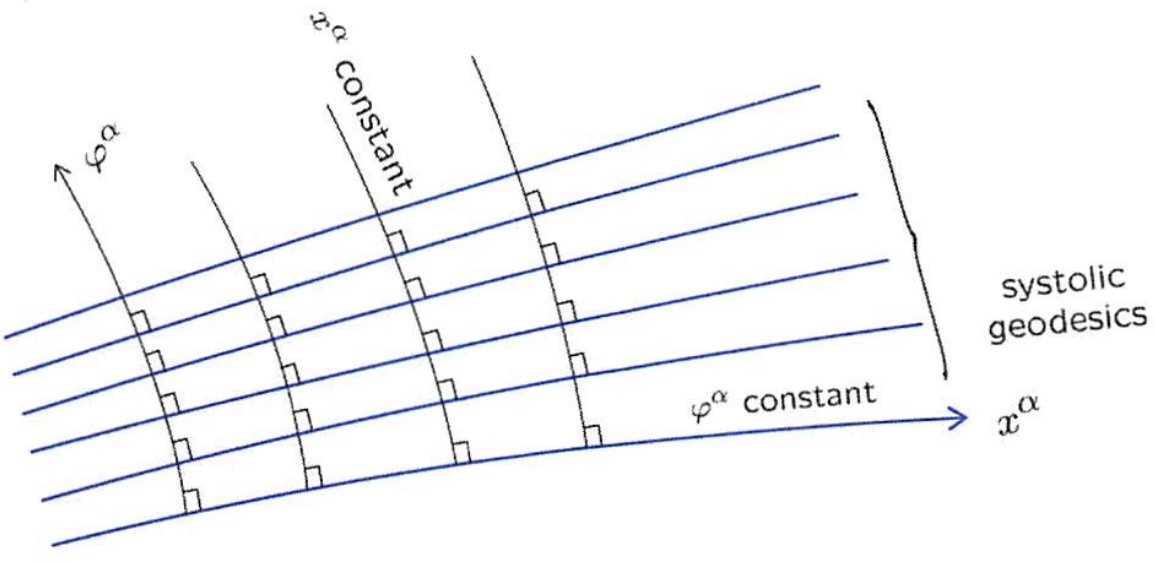

Figure 9: Normal coordinates associated with a collection of $\alpha$-geodesics (in blue). The coordinate $x^{\alpha}$ is a length parameter along the geodesics and the second coordinate $\varphi^{\alpha}$ runs normal to them. The geodesics are curves of constant $\varphi^{\alpha}$ while the curves orthogonal to them are curves of constant $x^{\alpha}$.

For this metric the Gaussian curvature $K$ can be calculated using equation (A.16) and the result is:

$$
K=-h_{\alpha} \frac{\partial^{2}}{\left(\partial x^{\alpha}\right)^{2}} \frac{1}{h_{\alpha}} .
$$

As a consistency check, the relation (7.14) between $u^{\alpha}$ and $\varphi^{\alpha}$,

$$
u^{\alpha}=-\frac{* d \varphi^{\alpha}}{\left|d \varphi^{\alpha}\right|}
$$

is satisfied in our construction. Indeed, a short calculation using (A.6) gives $* d \varphi^{\alpha}=-\left|h_{\alpha}\right| d x^{\alpha}$ and, given that $\left|d \varphi^{\alpha}\right|=\left|h_{\alpha}\right|$, the equality holds.

The expression (7.18) holds at a point $P$ for each class $C_{\alpha}$ of systolic geodesics that goes through $P$, and for each class one has $\left|d \varphi^{\alpha}\right|=\left|h_{\alpha}\right|$. Using these coordinates, our earlier constraint (7.11) on the sum of $d \varphi$ norms constrains the metric components in a simple way:

$$
\sum_{\alpha: d \varphi^{\alpha} \neq 0}\left|h_{\alpha}\right|=1
$$

This is a nontrivial constraint on the extremal metric.

The function $h_{\alpha}$ captures the density $\rho_{\alpha}$ of $\alpha$-geodesics. Consider two geodesics corresponding to $\varphi^{\alpha}=0$ and $\varphi^{\alpha}=\epsilon$, with $\epsilon$ infinitesimal. The distance between them is $\epsilon /\left|h_{\alpha}\right|$. We can use an arbitrarily small $\epsilon$ as a fixed $\varphi^{\alpha}$ interval that allows us to pick geodesics from the continuum and enumerate them. In particular with $\varphi^{\alpha} \in\left[0, \nu^{\alpha}\right]$ we get a total of $\nu^{\alpha} / \epsilon \alpha$-geodesics. It follows that the density $\rho_{\alpha}$ of $\alpha$-geodesics, defined as the number of geodesics per unit transverse length, is given by

$$
\rho_{\alpha}=\text { density of } \alpha \text {-geodesics }=\frac{1}{\epsilon /\left|h_{\alpha}\right|}=\frac{1}{\epsilon}\left|h_{\alpha}\right| .
$$

The constraint (7.21) now translates into

$$
\sum_{\alpha} \rho_{\alpha}=\frac{1}{\epsilon},
$$


meaning that at any point on the surface the sum of geodesic densities over all the classes that go through that point is the same.

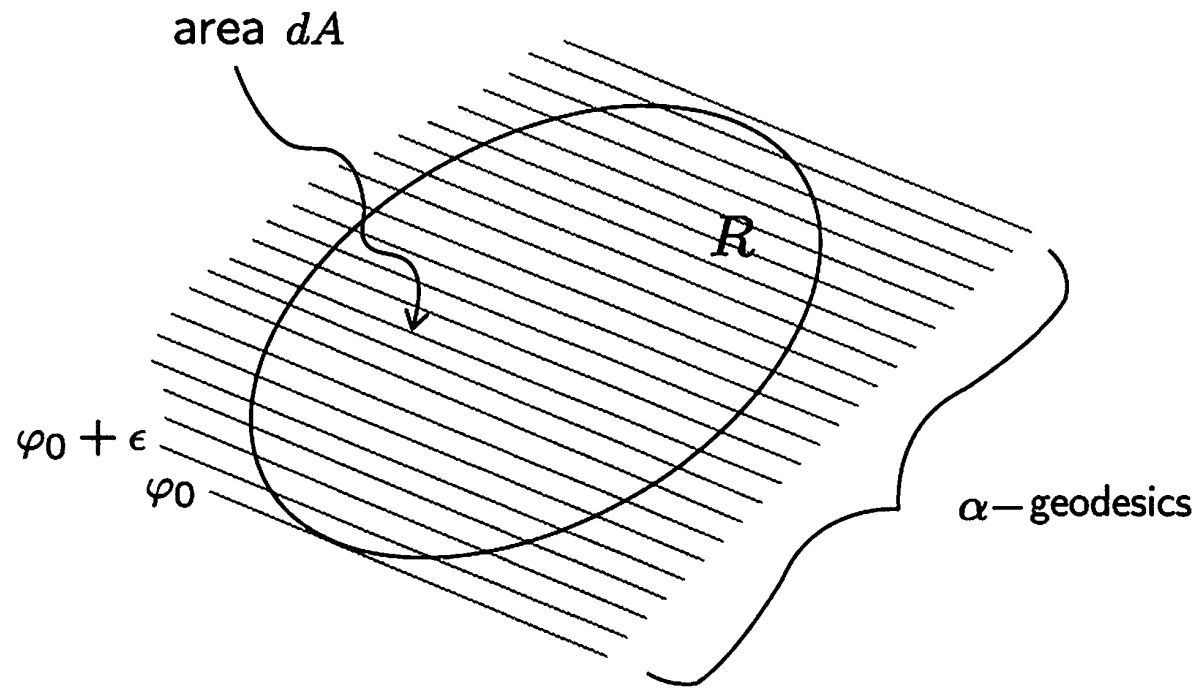

Figure 10: A set of $\alpha$-geodesics going through a tiny region $R$ of area $d A$. The geodesics are lines of constant $\varphi^{\alpha}$ values that differ by $\epsilon$. The area $d A$ can be expressed in terms of the length of all $\alpha$-geodesic segments going through the region.

We now explain that Eq. (7.23) stating that the sum of densities $\rho_{\alpha}$ is a constant over the surface is the local version of the formula (7.8) giving the area of the extremal metric as a sum of products $\nu^{\alpha} \ell_{\alpha}$. Indeed, we can relate the two formulas by computing the total length of all the (denumerable) $\alpha$-geodesics on the surface in two different ways. Consider first a small region $R$ of area $d A$, small enough that all metric components are accurately constant over $R$. By picking $\epsilon$ sufficiently small we can have a large number of $\alpha$-geodesics going through $R$. The area $d A$ is given by the total length $d L_{\alpha}(R)$ of the $\alpha$-geodesic segments within the region $R$, times the separation $\epsilon /\left|h_{\alpha}\right|$ between the geodesics (see figure 10):

$$
d A=d L_{\alpha}(R) \frac{\epsilon}{\left|h_{\alpha}\right|} \quad \Rightarrow \quad d L_{\alpha}(R)=\rho_{\alpha} d A .
$$

Let $d L(R) \equiv \sum_{\alpha} d L_{\alpha}(R)$ denote the total length of all geodesics in the small region $R$. Summing over $\alpha$ in the above equation and using (7.23) we find

$$
d L(R)=\frac{1}{\epsilon} d A .
$$

The total length of the geodesics contained within a region depends only on its area! Integrating now over the whole surface $M$, we find that the total length $L(M)$ of all the geodesics is the total area of the surface divided by $\epsilon$ :

$$
L(M)=\frac{1}{\epsilon} A .
$$

On the other hand, the number of $\alpha$-geodesics is $\nu^{\alpha} / \epsilon$, since $\varphi^{\alpha}$ changes monotonically by $\nu^{\alpha}$ over the full set of $\alpha$-geodesics. Each $\alpha$-geodesic has length $\ell_{\alpha}$, so their total length is $\ell_{\alpha} \nu^{\alpha} / \epsilon$. Summing over $\alpha$ :

$$
L(M)=\sum_{\alpha} \ell_{\alpha} \nu^{\alpha} / \epsilon=\frac{1}{\epsilon} \sum_{\alpha} \ell_{\alpha} \nu^{\alpha} .
$$


Equating the right-hand sides of the two equations above we recover the area formula (7.8).

Consider now the case when there is only a single set of systolic geodesics going through the points in a given region of the surface $M$. Then the constraint (7.21), having just one term, fixes $h_{\alpha}=1$. The metric is thus completely determined, and in fact is flat:

$$
d s^{2}=\left(d x^{\alpha}\right)^{2}+\left(d \varphi^{\alpha}\right)^{2} .
$$

This is a simple derivation of a previously known result that had rather intricate proofs $[25,26]$. In a region containing more than one set of systoles, the sum rule (7.21) still provides a strong constraint on the geometry, but it is less obvious what the general solution is.

Suppose we have exactly two types of systolic geodesics, arising from calibrations $u^{\alpha}$ and $u^{\beta}$ $(\beta \neq \alpha)$, going through the points in a given region of the surface. We then have

$$
d s^{2}=\left(d x^{\alpha}\right)^{2}+\frac{1}{h_{\alpha}^{2}}\left(d \varphi^{\alpha}\right)^{2}, \quad \text { and } \quad d s^{2}=\left(d x^{\beta}\right)^{2}+\frac{1}{h_{\beta}^{2}}\left(d \varphi^{\beta}\right)^{2} .
$$

We also have sum rule constraint:

$$
\left|h_{\alpha}\right|+\left|h_{\beta}\right|=1 \text {. }
$$

Another coordinate system gives additional insight. Using coordinates $x^{\alpha}$ and $x^{\beta}$ and recalling that $\left|d x^{\alpha}\right|=\left|d x^{\beta}\right|=1$ the inverse metric takes the form

$$
g^{-1}=\left(\begin{array}{ll}
1 & f \\
f & 1
\end{array}\right), \quad f=\left\langle d x^{\alpha}, d x^{\beta}\right\rangle=\left\langle\hat{u}^{\alpha}, \hat{u}^{\beta}\right\rangle .
$$

Here we learn something interesting. If the angle between the $\alpha$ and $\beta$ systoles is a constant, $g^{-1}$ is a constant matrix and the metric is flat. In $\left(x^{\alpha}, x^{\beta}\right)$ coordinates the metric reads

$$
d s^{2}=\frac{1}{1-f^{2}}\left[\left(d x^{\alpha}\right)^{2}-2 f d x^{\alpha} d x^{\beta}+\left(d x^{\beta}\right)^{2}\right] .
$$

In this oblique coordinate system, the vector $\frac{\partial}{\partial x^{\alpha}}$ is orthogonal to the $\beta$ systoles and the vector $\frac{\partial}{\partial x^{\beta}}$ is orthogonal to the $\alpha$ systoles. We have not been able to 'solve' the constraint (7.30) to find a parameterization of the allowed metrics in a two-band region. Our results in [22] indicate that both positive and negative Gaussian curvature are possible.

We conclude this section with some remarks on geodesic bands, namely bands formed by a continuous collection of $\alpha$-geodesics for some $\alpha$. Since these geodesics are closed curves of constant $\varphi^{\alpha}$, a geodesic band can be described as collection of homotopic geodesics in some range $\varphi^{\alpha} \in[a, b]$. A geodesic band is thus an annulus whose two boundaries are geodesics. An annulus has Euler number $\chi=2-b=0$, since the number $b$ of boundaries is equal to two. Since the boundary curves of the geodesic band are geodesics, the Gauss-Bonnet formula does not receive contributions from the boundary, and we conclude that

The integral of the Gaussian curvature over any geodesic band vanishes.

We can readily show that our earlier results confirm this explicitly. Over a geodesic band $\mathcal{B}$ with $\varphi^{\alpha} \in[a, b]$ the metric takes the form (7.18) with Gaussian curvature (7.19). We therefore have

$$
\int_{\mathcal{B}} K \sqrt{g} d^{2} x=\int_{a}^{b} d \varphi^{\alpha} \int_{0}^{\ell_{s}} d x^{\alpha} \frac{1}{h_{\alpha}} K=-\int_{a}^{b} d \varphi^{\alpha} \int_{0}^{\ell_{s}} d x^{\alpha} \frac{\partial^{2}}{\partial x^{\alpha 2}} \frac{1}{h_{\alpha}}=0
$$


since the geodesics are closed curves and $h_{\alpha}$ is single-valued.

For a band that does not intersect any other bands, the metric is flat (see (7.28)), so the above claim is trivially satisfied. For example, on a genus $g$ Riemann surface where the minimal-area metric arises from a quadratic differential, there is a single systolic geodesic almost everywhere on the surface, so the metric is flat almost everywhere. The negative curvature needed from the topology is given by delta functions at the zeroes of the quadratic differential. In general, however, the metric will not arise from a quadratic differential and we expect regions with one systolic band, regions with two systolic bands, and so on and so forth. There may be curvature over regions with two or more bands, but then on each band there must be both positive and negative curvature so that integrated curvature vanishes.

Focusing on a single band $R_{\alpha}$ of geodesics in the class $C_{\alpha}$ we can use the metric (7.18) to define the height $b^{\alpha}(x)$ of the band as a function of the position $x^{\alpha}$ along the band:

$$
b^{\alpha}(x) \equiv \int_{0}^{\nu^{\alpha}} \frac{d \varphi^{\alpha}}{\left|h_{\alpha}(x, \varphi)\right|} .
$$

The height $b_{\alpha}(x)$ is the distance between the boundary geodesics of the band at $x^{\alpha}$ measured along the segment $I\left(x^{\alpha}\right)=\left(x^{\alpha}, \varphi^{\alpha} \in\left[0, \nu^{\alpha}\right]\right)$ perpendicular to the geodesics at $x^{\alpha}$. Assume now there is a value $x_{0}^{\alpha}$ of $x^{\alpha}$ for which $I\left(x_{0}^{\alpha}\right) \subset U_{1}$, that is it lies the region where $R_{\alpha}$ is the only systolic band. Then $\left|h_{\alpha}\right|=1$ along this segment and

$$
b_{\alpha}\left(x_{0}^{\alpha}\right)=\nu^{\alpha}
$$

This gives simple characterization of $\nu^{\alpha}$ : it is the height of the band at a region where it is the only band on the surface. It is also clear that due to the sum rule (7.21) in general we have

$$
b_{\alpha}(x) \geq \nu_{\alpha}
$$

If we call $A_{\alpha}$ the area of the band $R_{\alpha}$ we have

$$
A_{\alpha}=\int_{0}^{\ell_{\alpha}} \int_{0}^{\nu^{\alpha}} \frac{d x^{\alpha} d \varphi^{\alpha}}{\left|h_{\alpha}\right|} \geq \nu^{\alpha} \ell_{\alpha}
$$

This provides a lower bound for $A_{\alpha}$. Let $M_{\alpha}$ denote the modulus of the band, or ring domain, $R_{\alpha}$. As noted in (6.11), the minimal area of a ring domain whose core curves are longer than or equal to $\ell$ is $\ell^{2} M$, where $M$ is the modulus of the ring domain. This gives us a second lower bound for $A_{\alpha}$ :

$$
A_{\alpha} \geq \ell_{\alpha}^{2} M_{\alpha}
$$

It would be interesting to see how the two lower bounds above compare. In other words: How does $M_{\alpha}$ compare with the ratio $\nu^{\alpha} / \ell_{\alpha}$ ?

\subsection{Properties of the solution from the dual}

Our aim in this subsection is to get more intuition for the dual program by showing how it enforces certain properties of the solution. These properties, having to do with interactions between bands of level sets, can also be understood from the primal.

Unlike in subsections $7.1-7.3$, here a configuration $\left(\nu^{\alpha}, \varphi^{\alpha}\right)$ is not necessarily a solution. 


\subsubsection{Segregation of level sets}

As we've seen, on a solution the level sets for the function $\varphi^{\alpha}$ are the $\alpha$-geodesics. The first lemma we prove essentially says that geodesic bands do not overlap unless they are forced to do so topologically.

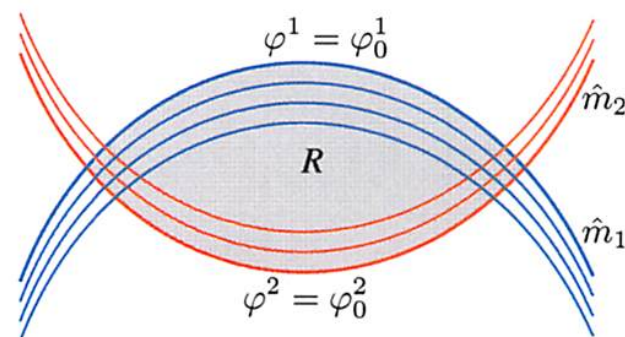

(a)

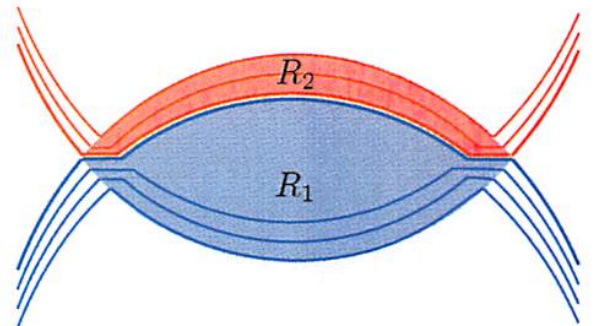

(b)

Figure 11: Illustration of the segregation lemma. (a) The region $R$ (gray) is bounded by the $\varphi^{1}$ level set $\hat{m}_{1}$ on which $\varphi^{1}=\varphi_{0}^{1}$ and the $\varphi^{2}$ level set $\hat{m}_{2}$ on which $\varphi^{2}=\varphi_{0}^{2}$, and contains overlapping bands of level sets. The blue curves are $\varphi^{1}$ level sets and the red curves are $\varphi^{2}$ level sets. (b) The level sets of the new configuration $\varphi^{1,2 \prime}$ and regions $R_{1}$ (light blue) and $R_{2}$ (pink). On $R_{2}$, $\varphi^{1 \prime}(x)=\varphi_{0}^{1}$, so there are no $\varphi^{1 \prime}$ level sets in $R_{2}$, and similarly with 1 and 2 switched. Note how the blue and red level sets that cross in (a) segregate and do not cross in (b).

Given a configuration in which a band of level sets of $\varphi^{1}$ and $\varphi^{2}$ overlap within some region $R$ bounded by a level set of $\varphi^{1}$ and a level set of $\varphi^{2}$ (see Figure 11(a)), we will show that there exists a configuration which is the same outside of $R$, but in which they don't overlap in $R$, and in which the dual objective,

$$
2 \sum_{\alpha} \nu^{\alpha} \ell_{\alpha}-\int_{M} \omega_{0}\left(\sum_{\alpha}\left|d \varphi^{\alpha}\right|_{0}\right)^{2},
$$

is at least as large as in the original configuration (see Figure 11(b)). (This holds even if level sets of other $\varphi^{\alpha}$ s also pass through $R$.) Therefore, in solving the dual program, we can restrict ourselves to configurations without such overlaps, in other words configurations where the level sets segregate. (However, it should be noted that imposing this constraint makes the dual program non-convex.) Bands of geodesics can, however, meet along a mutual boundary, which coincides with the last geodesic of each band.

Lemma 1 (Segregation). We consider the dual program in the form (5.7), with $\alpha$ taking at least two values, including 1 and 2 . Let $\left(\varphi^{\alpha}\right),\left(\nu^{\alpha}\right)$ be a feasible configuration, $R$ a region of $M$ (with no restriction on its topology), and $\varphi_{0}^{1}, \varphi_{0}^{2}$ constants such that:

(1) $R$ does not intersect the curves $m_{1}, m_{2}$ on which $\varphi^{1}, \varphi^{2}$ jump;

(2) at every point of $\partial R, \varphi^{1}=\varphi_{0}^{1}$ or $\varphi^{2}=\varphi_{0}^{2}$ (or both).

Then there exist regions $R_{1}, R_{2} \subseteq R$ and functions $\varphi^{1 \prime}, \varphi^{2 \prime}$ on $R$ such that

(a) $R_{1} \cup R_{2}=R$;

(b) $\varphi^{1 \prime}=\varphi_{0}^{1}$ on $R_{2}$ and $\varphi^{2 \prime}=\varphi_{0}^{2}$ on $R_{1}$; 
(c) $\varphi^{1 \prime}=\varphi^{1}$ and $\varphi^{2 \prime}=\varphi^{2}$ on $\partial R$;

(d) the dual objective with $\varphi^{1}$ replaced by $\varphi^{1 \prime}$ and $\varphi^{2}$ replaced by $\varphi^{2 \prime}$ within $R$ (and other $\varphi^{\alpha} \mathrm{S}$ and all $\nu^{\alpha}$ s unchanged) is at least as large as for the original configuration.

Note that, since $\varphi^{1,2 \prime}$ agrees with $\varphi^{1,2}$ on $\partial R$, the new configuration does not introduce any discontinuities on $\partial R$ and obeys the boundary conditions for $\varphi^{\alpha}$ on $\partial M$, and is therefore feasible.

Proof: It is easy to check that the function $f: \mathbb{R}^{2} \rightarrow \mathbb{R}$ defined by

$$
f\left(y_{1}, y_{2}\right):= \begin{cases}y_{1}-\operatorname{sgn}\left(y_{1} y_{2}\right) y_{2}, & \left|y_{1}\right|>\left|y_{2}\right| \\ 0, & \left|y_{1}\right| \leq\left|y_{2}\right|\end{cases}
$$

is continuous and has gradient either 0 or $(1, \pm 1)$ everywhere. Define $R_{1,2}$ as follows,

$$
\begin{aligned}
& R_{1}=\left\{x \in R:\left|\varphi^{1}(x)-\varphi_{0}^{1}\right| \geq\left|\varphi^{2}(x)-\varphi_{0}^{2}\right|\right\} \\
& R_{2}=\left\{x \in R:\left|\varphi^{1}(x)-\varphi_{0}^{1}\right| \leq\left|\varphi^{2}(x)-\varphi_{0}^{2}\right|\right\}
\end{aligned}
$$

and set

$$
\begin{aligned}
& \varphi^{1 \prime}(x)=\varphi_{0}^{1}+f\left(\varphi^{1}(x)-\varphi_{0}^{1}, \varphi^{2}(x)-\varphi_{0}^{2}\right), \\
& \varphi^{2 \prime}(x)=\varphi_{0}^{2}+f\left(\varphi^{2}(x)-\varphi_{0}^{2}, \varphi^{1}(x)-\varphi_{0}\right.
\end{aligned}
$$

Properties (a), (b), (c) are easy to check. We now consider the effect on the objective of replacing $\varphi^{1,2}$ with $\varphi^{1,2 \prime}$. On $R_{1}$, we have

$$
\varphi^{\prime 1}(x)=\varphi^{1}(x) \pm\left(\varphi^{2}(x)-\varphi_{0}^{2}\right), \quad \varphi^{2 \prime}(x)=\varphi_{0}^{2},
$$

and therefore,

$$
d \varphi^{\prime 1}=d \varphi^{1} \pm d \varphi^{2}, \quad d \varphi^{2 \prime}=0
$$

so by the triangle inequality

$$
\left|d \varphi^{\prime 1}\right|_{0}+\left|d \varphi^{\prime 2}\right|_{0}=\left|d \varphi^{1} \pm d \varphi^{2}\right|_{0} \leq\left|d \varphi^{1}\right|_{0}+\left|d \varphi^{2}\right|_{0} .
$$

By the same reasoning with 1 and 2 switched, (7.45) applies also in $R_{2}$. Therefore the integrand in the second term of the dual objective is not increased:

$$
\left(\left|d \varphi^{1 \prime}\right|_{0}+\left|d \varphi^{2 \prime}\right|_{0}+\sum_{\alpha \neq 1,2}\left|d \varphi^{\alpha}\right|_{0}\right)^{2} \leq\left(\sum_{\alpha}\left|d \varphi^{\alpha}\right|_{0}\right)^{2} .
$$

Since the $\varphi^{\alpha}$ s outside of $R$ and the $\nu^{\alpha}$ s are unchanged, the dual objective is not decreased. This establishes property (d).

The objective will in fact increase under the substitution $\varphi^{1,2} \rightarrow \varphi^{1,2 \prime}$, unless everywhere in $R$ the triangle inequality in (7.45) is saturated, which requires that $d \varphi^{1}$ and $d \varphi^{2}$ be parallel (or antiparallel) wherever they are both non-zero. Since the tangent vector of a level set of $\varphi^{1,2}$ is given by $\epsilon^{\mu \nu} \partial_{\nu} \varphi^{1,2}$, this in turn requires that any $\varphi^{1}$ level set and any $\varphi^{2}$ level set must fully coincide within $R$. (More precisely, connected components within $R$ must fully coincide.) Thus in a situation like the one shown in Figure 11(a), in which the level sets of $\varphi^{1}$ and $\varphi^{2}$ intersect transversely, the objective can be increased, so such a situation cannot occur in a solution. 
The fact that a configuration like the one in Figure 11(a) cannot occur in a solution can also be understood from the primal program. Specifically, we can easily show that a region $R$ cannot be bounded by the union of a segment $\gamma_{1}$ of a 1-geodesic $\hat{m}_{1}$ and a segment $\gamma_{2}$ of a 2-geodesic $\hat{m}_{2}$. Since together they bound a region, $\gamma_{1}$ and $\gamma_{2}$ are homologous (relative to their intersection points). Therefore, replacing $\gamma_{1}$ with $\gamma_{2}$ on $\hat{m}_{1}$ and $\gamma_{2}$ with $\gamma_{1}$ on $\hat{m}_{2}$ gives an element $\tilde{m}_{1}$ of the class $C_{1}$, that must therefore have length at least $\ell_{1}$, and an element $\tilde{m}_{2}$ of the class $C_{2}$ that must therefore have length at least $\ell_{2}$. On the other hand, since we traded pieces of curves, the total length is unchanged,

$$
\operatorname{length}\left(\tilde{m}_{1}\right)+\operatorname{length}\left(\tilde{m}_{2}\right)=\operatorname{length}\left(\hat{m}_{1}\right)+\operatorname{length}\left(\hat{m}_{2}\right)=\ell_{1}+\ell_{2},
$$

so $\tilde{m}_{1}$ and $\tilde{m}_{2}$ must have length equal to $\ell_{1}$ and $\ell_{2}$, respectively. They must therefore be 1,2 -geodesics respectively. But this is impossible since they have corners.

\subsubsection{Cylinder versus pants}

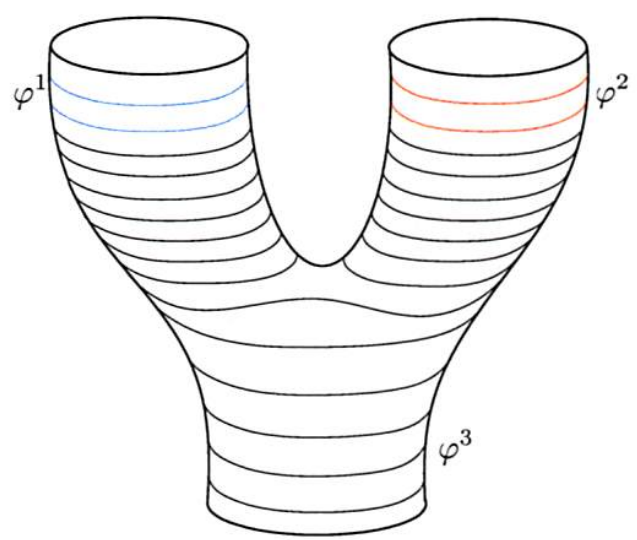

(a)

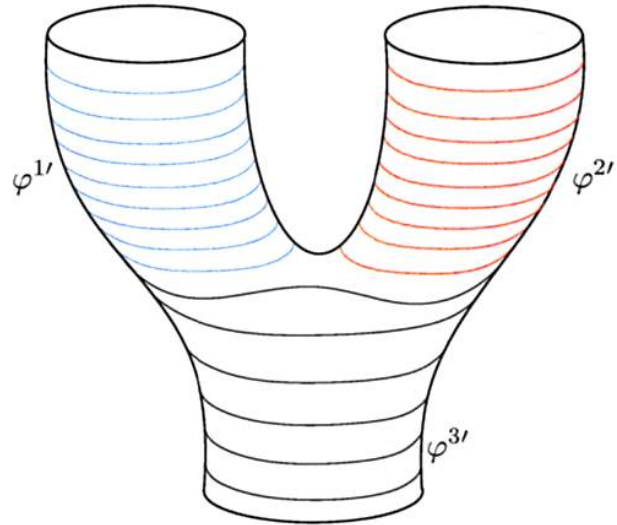

(b)

Figure 12: (a) Pants diagram with three types of level sets, corresponding to $\varphi^{1}, \varphi^{2}$, and $\varphi^{3}$. Above the crotch the $\varphi^{3}$ level set is the union of representatives of $C_{1}$ and $C_{2}$. (b) The same diagram, where the $\varphi^{3}$ level sets above the crotch now belong to the $\varphi^{1}$ and $\varphi^{2}$ level sets. This rearrangement of curves leads to a larger objective in the dual program when the length parameters satisfy $\ell_{1}+\ell_{2}>\ell_{3}$.

Even without overlapping, bands of level sets can still interact, in a sense we will now explain. Consider a situation like that illustrated in Figure 12, which shows a "pair of pants" surface; this could be part of a larger surface. We consider three homology cycles, $C_{1,2,3}$, represented by the top left, top right, and bottom boundaries respectively. In Figure 12(a), we have a band of level sets in each class, and these bands don't overlap. Above the crotch, each $\varphi^{3}$ level set is the union of a representative of $C_{1}$ and a representative of $C_{2}$. In Figure 12(b), the configuration has been changed so that those curves are level sets of $\varphi^{1 \prime}$ and $\varphi^{2 \prime}$, and not of $\varphi^{3 \prime}$. Correspondingly, in order for the new configuration to obey the boundary and jump conditions, we change the $\nu^{\alpha}$ values:

$$
\nu^{1 \prime}=\nu^{1}+\Delta \nu, \quad \nu^{2 \prime}=\nu^{2}+\Delta \nu, \quad \nu^{3 \prime}=\nu^{3}-\Delta \nu,
$$


where $\Delta \nu$ is the "number" of level sets that have been transferred. We have

$$
\sum_{\alpha=1}^{3}\left|d \varphi^{\alpha \prime}\right|=\sum_{\alpha=1}^{3}\left|d \varphi^{\alpha}\right|,
$$

so the second term in the dual objective (7.39) is unchanged (even if there are level sets of other $\varphi^{\alpha}$ s passing through this region). However, by (7.48), the first term changes by

$$
2\left(\ell_{1}+\ell_{2}-\ell_{3}\right) \Delta \nu .
$$

So, if the $\ell_{\alpha}$ obey the strict triangle inequality

$$
\ell_{3}<\ell_{1}+\ell_{2},
$$

then the objective increases. In effect, the $\varphi^{1}$ and $\varphi^{2}$ bands "eat up" the $\varphi^{3}$ band, until no $\varphi^{3}$ level sets remain that are the union of an element of $C_{1}$ and of $C_{2}$. So, in solving the dual, we can restrict our attention to bands that are cylindrical and not pairs of pants. (However, imposing this constraint makes the dual non-convex.)

If the length constraints obey the inequality (7.51), the fact that no 3-geodesic is a union of a $C_{1}$ representative and a $C_{2}$ representative also follows directly from the primal. Indeed, any 3 -geodesic must have length $\ell_{3}$, but by the length conditions on $C_{1}$ and $C_{2}$ representatives, the composite one will be longer, at least of length $\ell_{1}+\ell_{2}$, showing this is not possible. Our purpose here was to show how the dual program enforces this fact.

\subsection{Quadratic differentials and the dual objective}

Jenkins and Strebel proved the following result (Theorem 21.10 [4]): Let $M$ be a Riemann surface with a set of admissible curves $\gamma_{1}, \ldots, \gamma_{k}$, that is, a set of nontrivial-homotopy, non-homotopic, simple closed Jordan curves that have non-intersecting representatives. Now let $R_{i}$ with $i=1, \ldots, k$, be disjoint ring domains (annuli) of homotopy type $\gamma_{i}$ and let $M_{i}$ be the modulus of $R_{i}$. To each curve $\gamma_{i}$ we assign a positive number $\ell_{i}$. Consider now the functional $\mathcal{F}$ defined by

$$
\mathcal{F}=\ell_{1}^{2} M_{1}+\ldots \ell_{k}^{2} M_{k}=\sum_{i=1}^{k} \ell_{i}^{2} M_{i} .
$$

Maximizing $\mathcal{F}$ over the shape of the disjoint ring domains gives

$$
\operatorname{Max}_{R_{i}} \mathcal{F}=\|\Phi\|,
$$

that is, the norm of a Jenkins-Strebel quadratic differential $\Phi(z) d z^{2}$ on the surface $M$. The $R_{i}$ are the characteristic ring domains of the quadratic differential $\Phi$ which has closed trajectories. Strebel and Jenkins also show that the norm of the quadratic differential is equal to the minimal area of a metric in $M$ such that any curve homotopic to $\gamma_{i}$ is longer than or equal to $\ell_{i}$. Thus the maximum of the objective $\mathcal{F}$ coincides with the minimum of the objective in the homotopy minimal area problem.

We want to understand how the objective $\mathcal{F}$ arises in the context of the dual program. Of course it will not arise in general situations when one constrains classes of curves that have intersections; the objective in the dual program is a subtle generalization of $\mathcal{F}$ when the curves do not form 
an admissible set. When they do, the dual program objective can be related to $\mathcal{F}$. To discuss the relation consider a setup where the homology program is equivalent to the homotopy problem above.

In this setup we choose an admissible set of curves $\gamma_{1}, \cdots, \gamma_{k}$ all of which are also nontrivial curves in homology classes $C_{1}, \cdots C_{k}$. We then impose the length conditions $\ell_{1}, \cdots, \ell_{k}$ on the respective classes of curves. Since all curves homotopic to $\gamma_{i}$ are also homologous to $\gamma_{i}$ the dual program imposes all the conditions of the homotopy program. Moreover, by suitably choosing the length parameters $\ell_{i}$ it is possible to guarantee that, at least in some cases, the homology program does not impose additional conditions absent in the homotopy program.

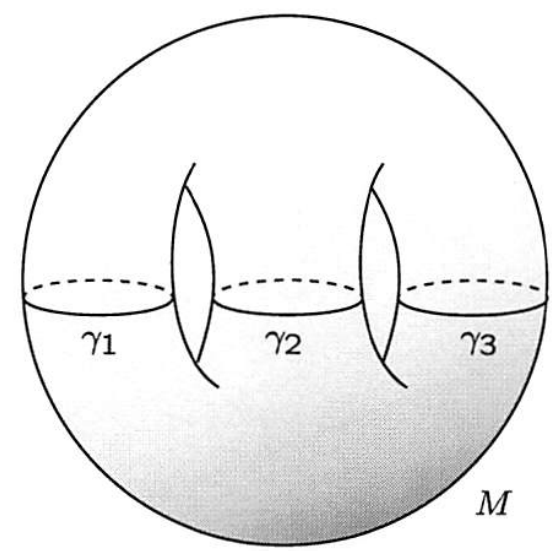

Figure 13: A genus two surface with a maximal admissible set of curves $\gamma_{1}, \gamma_{2}, \gamma_{3}$ of nontrivial homotopy. The curves are also of nontrivial homology, and with suitable length conditions the dual program and the homotopy minimal area problem are equivalent.

This is illustrated in the genus-two surface $M$ shown in Figure 13, and equipped with an admissible set of curves $\gamma_{1}, \gamma_{2}, \gamma_{3}$. The set is maximal: any curve on a different homotopy class would intersect at least one of the curves in the set. The curves are also nontrivial in homology, belonging to classes denoted as $C_{1}, C_{2}$, and $C_{3}$. The curves are related in homology. Up to signs determined by the choice of orientations, we have relations of the form

$$
\gamma_{i} \sim \pm \gamma_{j} \pm \gamma_{k}, \quad i \neq j \neq k,
$$

for all values of $i, j, k$ in the set $1,2,3$. As a result, for example, the calibration that guarantees that the length of any curve homologous to $\gamma_{1}$ exceeds or equals $\ell_{1}$, will also imply that for curves $\gamma_{2}^{\prime}$ and $\gamma_{3}^{\prime}$ homotopic to $\gamma_{2}$ and $\gamma_{3}$, respectively, we have

$$
\text { length }\left(\gamma_{2}^{\prime}\right)+\operatorname{length}\left(\gamma_{3}^{\prime}\right) \geq \ell_{1} .
$$

This will impose no new condition if $\ell_{2}+\ell_{3} \geq \ell_{1}$. Considering the other homology relations, we need the length parameters to satisfy

$$
\ell_{i}+\ell_{j} \geq \ell_{k}, \quad \forall i \neq j \neq k .
$$

We can argue that these inequalities, which are easily satisfied, guarantee that no new condition is imposed by the homology problem. Indeed, consider a general representative $m_{1}$ of $C_{1}$. Being a sum of closed curves, $m_{1}$ may include a curve in $C_{2}$ and/or a curve in $C_{3}$, as considered above, but it may contain other curves. It cannot contain a single curve in a new homotopy class, because our 
set is maximal and any new homotopy class must necessarily intersect one of the representatives in the set. This, however, cannot happen for a curve representing $C_{1}$.

Consider, therefore, any setup as above, where the homotopy and the homology programs are equivalent. Then the maximum of the dual objective is the minimum of the homotopy problem. As we know from the homotopy problem, the bands of saturating geodesics at the optimum form nonintersecting ring domains, or cylinders. This is consistent with the segregation lemma considered in the previous subsection as a property of the dual program.

It now follows that for any set of fixed non-overlapping ring domains $R_{1}, \ldots R_{k}$ of homotopy type $\gamma_{1}, \ldots \gamma_{k}$ and length parameters $\ell_{1}, \ldots, \ell_{k}$, the objective of the dual program will have an optimum that equals the sum $\sum_{i} \ell_{i}^{2} M_{i}$ where $M_{i}$ is the modulus of $R_{i}$. This follows from the calculation in section 6.1, that proved that the optimum of the dual objective in an annulus of modulus $M$ and length parameter $\ell$ is indeed $\ell^{2} M$. Constrained by the condition of non-overlapping ring domains, further maximization of the dual objective over the shape of the ring domains clearly becomes maximization of $\mathcal{F}$.

\section{Back to the homotopy problem}

We now return to the original minimal area problem of closed string field theory, which constrains all curves of nontrivial homotopy, and explain how it can be solved using the ideas considered in this paper. As a first step we describe the natural homological problem associated with the problem in homotopy. We then show that the homology problem does not impose extraneous length conditions. The main complication is that there usually are nontrivial homotopy closed curves $\gamma$ that are trivial in homology and thus their lengths cannot be constrained by calibrations. To deal with this we take the original surface $M$ and construct a covering space $\tilde{M}$ where the curve $\gamma$ becomes homologically nontrivial. In principle a double cover suffices and much of our discussion is couched in this language. We explain that a calibration $\tilde{u}$ on $\tilde{M}$, antisymmetric under the exchange of the two sheets, together with a metric symmetric under the exchange, correctly constrains the length of all curves homotopic to $\gamma$ in the original surface. By introducing such a double cover for each homotopy class of curves that is trivial in homology, the minimal area homotopy problem is solved in terms of homology and calibrations. The same double cover can also be used for the dual program by imposing suitable conditions on the functions $\varphi^{\alpha}$ or the one-forms $\eta^{\alpha}$.

\subsection{The homotopy problem reduced to homology}

As discussed in the introduction, the minimal area problem for closed string field theory $[7,8]$ asks for the conformal metric of least area on a Riemann surface under the condition that all non-contractible closed curves have length greater than or equal to 1 . We recall the formulation in (4.11):

$$
\begin{aligned}
& \text { Minimize } \int_{M} \omega_{0} \Omega \quad \text { over } \Omega \geq 0 \text { (function) } \\
& \text { subject to } \quad \ell_{s}-\int_{\gamma} \sqrt{\Omega}|\dot{x}|_{0} \leq 0, \forall \gamma \in \Gamma .
\end{aligned}
$$

Here $\Gamma$ is the set of all homotopically nontrivial simple closed curves $\gamma$ on $M$. It is known that this problem is not changed if we reduce the constraint space from $\Gamma$ down to a smaller set of nontrivial 
homotopy classes $S \subset \pi_{1}(M)$, where any class in $S$ has a Jordan closed curve representative. Given a class $D \in S$, the class $D^{-1}$, representing oppositely oriented curves, is not included in $S$. The minimal-area problem is now written as

$$
\begin{aligned}
& \text { Minimize } \int_{M} \omega_{0} \Omega \quad \text { over } \Omega \geq 0 \text { (function) } \\
& \text { subject to } \ell_{s}-\int_{\gamma} \sqrt{\Omega}|\dot{x}|_{0} \leq 0, \forall \gamma \in S .
\end{aligned}
$$

Consider the usual map $\Pi$ from homotopy to homology classes, $\Pi: \pi_{1}(M) \rightarrow H_{1}(M)$. The map is not one-to-one and has a kernel $S_{0}$. We can now split $S$ into two non-overlapping subsets

$$
S=S_{1} \cup S_{0}, \quad S_{0}:=\Pi^{-1}(0) .
$$

We write the elements of $S_{0}$ as $D_{0}^{k}$ (where $k$ is an index):

$$
\Pi\left(D_{0}^{k}\right)=0, \forall k .
$$

The representatives of $D_{0}^{k}$ are homotopically non-trivial closed curves that cut $M$ into two separate pieces, at least one of which has no other boundary. The elements of $S_{1}$, on the other hand, are homotopy classes $D_{\alpha}^{i}$ that are mapped by $\Pi$ to a nontrivial homology class $C_{\alpha}$ :

$$
\Pi\left(D_{\alpha}^{i}\right)=C_{\alpha} \neq 0
$$

The index $i$ on the homotopy class is needed because curves in different homotopy classes may be homologous. We call $W$ the image of $S_{1}$ under the map $\Pi$

$$
W=\Pi\left(S_{1}\right)=\left\{C_{\alpha}, \alpha \in J_{0}\right\}
$$

where we introduced the index set $J_{0}$ that labels all the homology classes in $W$. By definition, the homology classes in $W$ all have representatives that are simple closed Jordan curves.

The natural homological problem associated to the homotopy problem constrains the homology classes $C_{\alpha} \in W$. Using calibrations, the program is just the primal MAP, version 1, given in (4.32), for the appropriate set of classes and with length constraint $\ell_{\alpha}=\ell_{s}$ for all classes:

$$
\begin{aligned}
& \text { Minimize } \int_{M} \sqrt{g^{0}} \Omega \text { over } \Omega \text { (function), } u^{\alpha} \text { (one-forms) } \\
& \text { subject to: } \quad\left|u^{\alpha}\right|_{0}^{2}-\Omega \leq 0 \text {, } \\
& d u^{\alpha}=0, \\
& \ell_{s}-\int_{m_{\alpha}} u^{\alpha}=0, \forall \alpha \in J_{0} \text {. }
\end{aligned}
$$

This program will have to be supplemented to solve the original homotopy problem (8.1). The requisite program will be written at the end of the section.

This homology program, we claim, gives an optimum in which all curves in each homotopy class in $S_{1}$ satisfy the length condition. Indeed, any curve in a class in $S_{1}$ is itself a representative of a class in $W$, and its length is properly constrained by the homology problem. In fact, each class in $W$ effectively constraints all the classes in $S_{1}$ that map to it under $\Pi$. 
We now explain why the homology problem does not impose extraneous length conditions. Any class $C_{\alpha} \in W$, in addition to having representatives with a single closed curve that are properly constrained, also contains representatives with multiple closed curves, and the sum of their lengths is also constrained to be greater than or equal to $\ell_{s}$. Consider a $C_{\alpha}$ representative $m_{1}+\cdots+m_{k}$, with $k \geq 2$, where each $m_{i}(i=1, \ldots, k)$ is a simple closed curve. Since the original class $C_{\alpha}$ is nontrivial, not all of the $m$ 's can be trivial in homology. At least one $m_{i}$ must be a representative of a nontrivial homology class. That $m_{i}$ must then be a homotopically nontrivial simple closed curve. It follows that $m_{i}$ belongs to $S_{1}$ therefore has length greater than or equal to $\ell_{s}$. Since lengths add, the representative $m_{1}+\cdots+m_{k}$ is automatically guaranteed to satisfy the length condition. It does not impose a new constraint.

Note that the set $W$ contains an infinite number of homology classes. Constraining all of its members therefore requires an infinite number of calibrations $u^{\alpha}$. In practice, one would only constrain a finite number of cycles and check by hand that the rest of the cycles in $W$ do not contain any representatives with length less than $\ell_{s}$. If any cycles did, then the program would have to be re-solved including the offending cycles.

Since the curves in $S_{1}$ are properly constrained by the homology problem, this leaves the problem of the curves in $S_{0}$, homotopy nontrivial curves that are homologically trivial. The next subsection will address this issue.

\subsection{Homologically trivial curves}

One can only use calibrations to constrain the lengths of curves that are homologically non-trivial. The reason is that a calibration $u$ constrains the length of a curve $\gamma$ using the inequality

$$
\operatorname{length}(\gamma) \geq\left|\int_{\gamma} u\right|
$$

For this to work the period $\int_{\gamma} u$ must be non-zero. For a homologically trivial curve $\gamma$, however, we have $\gamma=\partial R$ and therefore

$$
\int_{\gamma} u=\int_{R} d u=0
$$

because calibrations are closed forms. We can get around this problem, however, by observing that, on a suitable multiple cover of the surface, a homologically trivial curve lifts to a homologically nontrivial one. Here we will explain how to construct a double cover with this property and thereby constrain the lengths of such curves by calibrations. By a straightforward generalization, it is also possible to construct a higher-multiplicity cover, and in certain cases this may be convenient, for example to preserve symmetries of the surface; we will see an example in [22].

\subsubsection{Defining a suitable covering space}

Let $D$ be a non-trivial homotopy class in the set $S_{0}$; this means that it has a representative $\gamma_{0}$ that is a homotopically non-trivial but homologically trivial Jordan closed curve on the surface $M$ (see Figure 14). Our goal now is to cut $M$ appropriately and glue the cut surface to another copy of itself to produce a covering surface $\tilde{M}$ on which $\gamma_{0}$ lifts to a homologically nontrivial curve $\tilde{\gamma}_{0}$. We do this cut as follows. 
Since $\gamma_{0}$ is homologically trivial, it must be the boundary of a region $R$ of $M: \partial R=\gamma_{0}$. The region $R$ has genus at least one, otherwise $\gamma_{0}$ would not be homotopically nontrivial. Let $\gamma_{R}$ be a Jordan curve that wraps one of the handles of $R$ and does not intersect $\gamma$ (see Figure 14). The surface $M$ is then cut along $\gamma_{R}$ without falling into two pieces. On the cut surface $M_{\text {cut }}$, denote by $\gamma_{R}^{+}$and $\gamma_{R}^{-}$the two boundaries generated by the cut along $\gamma_{R}$. We orient the curves $\gamma_{R}^{+}$and $\gamma_{R}^{-}$such that their sum is homologous to $\gamma_{0}$ :

$$
\gamma_{0} \sim \gamma_{R}^{+}+\gamma_{R}^{-} .
$$

On the other side of $\gamma_{0}$ we have the complement $R^{c}$ of $R$ in $M$. If the surface $M$ has a boundary component within $R^{c}$, as it is the case in Figure 14, then $\gamma_{0}$ is not homologically trivial within $R^{c}$ and we need not do any further cut. If $M$ has no boundary component, $\partial R^{c}=-\gamma$ and we must also cut $R^{c}$, which must have genus at least one. Just like we did for $R$, we select a handle and a Jordan curve $\gamma_{R^{c}}$ that wraps the handle and does not intersect $\gamma$. The surface is then cut along $\gamma_{R^{c}}$. We denote by $\gamma_{R^{c}}^{+}$and $\gamma_{R^{c}}^{-}$the two new boundaries generated by the cut along $\gamma_{R^{c}}$. The result is now the cut surface $M_{\text {cut }}$.
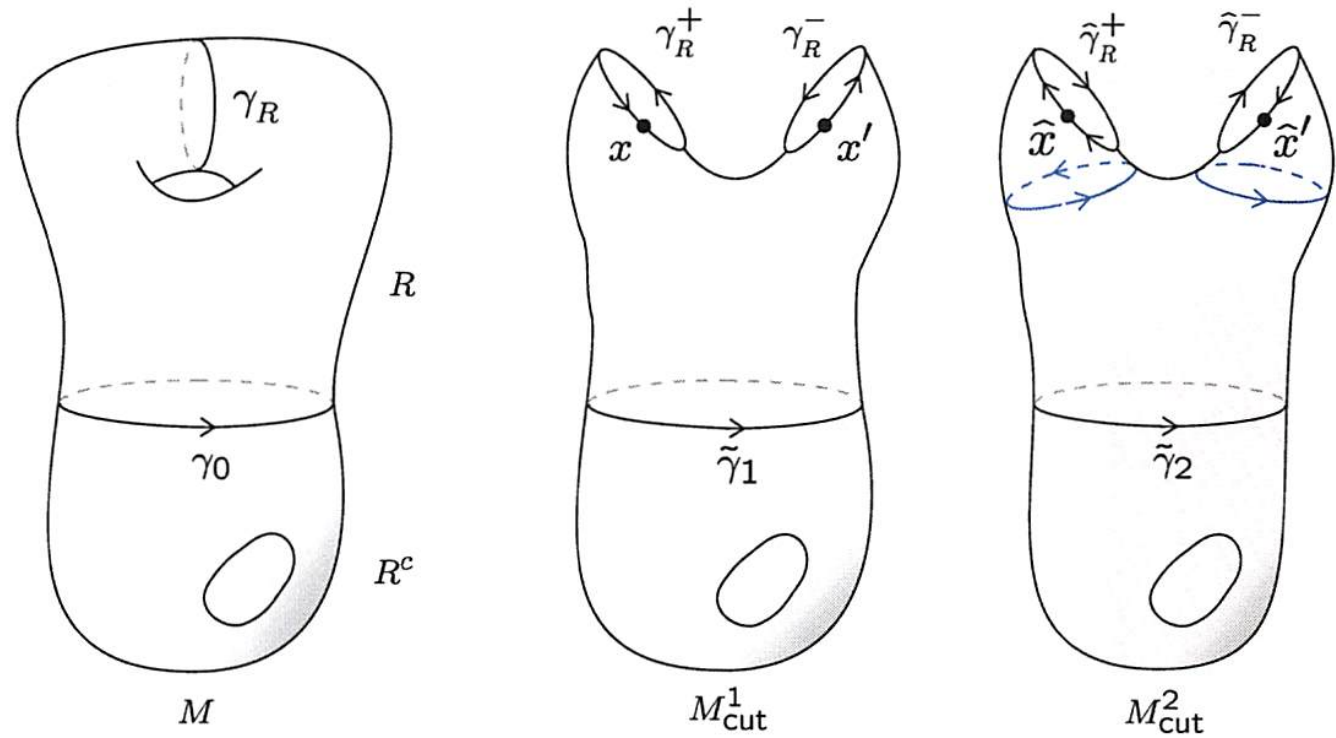

Figure 14: Left: The surface $M$ with a curve $\gamma_{0}$ in the nontrivial homotopy class $D$. The curve $\gamma_{0}$ is homologically trivial and divides $M$ into $R$ and $R^{c}$. We choose a curve $\gamma_{R}$ along the handle in $R$. Right: The surface $M$ is cut open along $\gamma_{R}$ and and it is then doubled to form $M_{\text {cut }}^{1}$ and $M_{\text {cut }}^{2}$. These two are glued to form $\tilde{M}$, with $\gamma_{R}^{+}$attached to $\hat{\gamma}_{R}^{-}$and $\gamma_{R}^{-}$attached to $\gamma_{R}^{+}$. The curves $\tilde{\gamma}_{1}$ and $\tilde{\gamma}_{2}$ are the images of $\gamma_{0}$ in the double surface $\tilde{M}$, and the blue curves (homologous to $\tilde{\gamma}_{2}$ ) help show that $\tilde{\gamma}_{1}=-\tilde{\gamma}_{2}$ in $H_{1}(\tilde{M})$. The curve $\tilde{\gamma}_{1}$ is homologically nontrivial in $\tilde{M}$.

Now consider two copies $M_{\text {cut }}^{1}$ and $M_{\text {cut }}^{2}$ of the cut surface $M_{\text {cut }}$. On $M_{\text {cut }}^{2}$ we define the analogous curves $\hat{\gamma}_{R}^{+}$and $\hat{\gamma}_{R}^{-}$but with opposite orientations as compared with $M_{\text {cut }}^{1}$.

We define $\tilde{M}$ as the double cover obtained as follows: $\gamma_{R}^{+}$on $M_{\text {cut }}^{1}$ is glued to $\hat{\gamma}_{R}^{-}$on $M_{\text {cut }}^{2}$ and $\gamma_{R}^{-}$on $M_{\text {cut }}^{1}$ is glued to $\hat{\gamma}_{R}^{+}$on $M_{\text {cut }}^{2}$ (see Figure 14 ):

$$
\gamma_{R}^{+} \sim \hat{\gamma}_{R}^{-}, \text {and } \gamma_{R}^{-} \sim \hat{\gamma}_{R}^{+} .
$$

With the gluing done consistent with the orientations shown in the figure, the above are relations in $H_{1}(\tilde{M})$. An analogous gluing is done for the cuts in the region $R^{c}$ if they exist. The projection 
map $p$ taking the double cover to the original surface,

$$
p: \tilde{M} \rightarrow M,
$$

is obvious from the construction. ${ }^{11}$

Since $\gamma_{0}$ does not intersect the cuts, its images $\tilde{\gamma}_{1}, \tilde{\gamma}_{2}$ on the first and second sheets of $\tilde{M}$ respectively are both closed curves. The objective of the construction is then achieved: $\tilde{\gamma}_{1}$ and $\tilde{\gamma}_{2}$ are nontrivial in $H_{1}(\tilde{M})$. Indeed, if we cut $\tilde{M}$ along $\tilde{\gamma}_{1}$, it is clear from the figure that $\tilde{M}$ will break into two pieces and, in each piece, $\tilde{\gamma}_{1}$ will be homologous to a boundary component and thus not trivial. If $R^{c}$ did not have a boundary, a cut and glue construction similar to that for $R$ would have been needed to build $\tilde{M}$, and a cut along $\tilde{\gamma}_{1}$ would have not split $\tilde{M}$ into two surfaces, again showing that $\tilde{\gamma}_{1}$ has nontrivial homology in $\tilde{M}$. The same line of argument holds for $\tilde{\gamma}_{2}$, which is also of nontrivial homology.

Noting that on $M_{\text {cut }}^{1}$ we have

$$
\tilde{\gamma}_{1} \sim \gamma_{R}^{+}+\gamma_{R}^{-}
$$

and on $M_{\text {cut }}^{2}$ we have

$$
\tilde{\gamma}_{2} \sim-\hat{\gamma}_{R}^{+}-\hat{\gamma}_{R}^{-}
$$

we conclude that

$$
\tilde{\gamma}_{1} \sim-\tilde{\gamma}_{2} .
$$

We define $\tilde{C} \in H_{1}(\tilde{M})$ to be the homology class of $\tilde{\gamma}_{1}$ :

$$
\tilde{\gamma}_{1} \in \tilde{C} \in H_{1}(\tilde{M}) .
$$

\subsubsection{Defining the calibration on $\tilde{M}$}

We can now apply our methods to the nontrivial cycle $\tilde{C}$ in $\tilde{M}$. By the same reasoning as in subsection 4.4 , there exists a calibration $\tilde{u}$ on $\tilde{M}$ such that $\int_{\tilde{C}} \tilde{u}=1$ if and only if every representative of $\tilde{C}_{1}$ has length at least 1 .

Given a metric on $M$, there is a unique way to lift it to one on $\tilde{M}$ that is invariant under exchange of the two sheets: the metric on $M$ is copied onto each of the cut surfaces $M_{\text {cut }}^{1}$ and $M_{\text {cut }}^{2}$ before they are glued. We also impose a symmetry condition on the calibration. Since $\tilde{\gamma}_{1}+\tilde{\gamma}_{2} \sim 0$ in homology, we have $\int_{\tilde{\gamma}_{2}} \tilde{u}=-\int_{\tilde{\gamma}_{1}} \tilde{u}$. Since $\tilde{\gamma}_{1}$ and $\tilde{\gamma}_{2}$ are exchanged under exchange of the sheets, we demand that $\tilde{u}$ is antisymmetric under sheet exchange. In fact, a slight generalization of the max flow-min cut theorem establishes the following statement: given a symmetric metric on $\tilde{M}$, there exists an antisymmetric calibration $\tilde{u}$ such that $\int_{\tilde{C}} \tilde{u}=1$ if and only if every representative of $\tilde{C}$ has length at least 1.

We now show that the antisymmetry of $\tilde{u}$ under exchange of the two sheets implies that $\tilde{u}$ on $M_{\text {cut }}^{1}$ is anti-continuous across the cut: it takes opposite values on points immediately across the

\footnotetext{
${ }^{11}$ We can characterize $\tilde{M}$ recalling that (see [35] Ch. 3, Theorem 4) covering spaces can be associated to subgroups of the first homotopy group: For $X$ a connected space and $x \in X$, let $H$ be a subgroup of $\pi_{1}(X, x)$. Then there is a covering space $(\tilde{X}, p)$ and a point $\tilde{x} \in \tilde{X}$ such that $p(\tilde{x})=x$ and $\pi_{1}(\tilde{X}, \tilde{x})$, when pulled back to $X$, equals $H$. In our case, $\tilde{M}$ is associated to the subgroup $H$ of $\pi_{1}(M, x)$ consisting of homotopy classes of curves that intersect $\gamma_{R}$ an even number of times. These are precisely the curves in $M$ that descend from closed curves on $\tilde{M}$.
} 
cut. Because of the antisymmetry of the calibration, we have the following relations among the calibration $\tilde{u}$ at points on the two sheets that map to the same point on $M$ (see Figure 14):

$$
\tilde{u}(x)=-\tilde{u}(\hat{x}), \quad \tilde{u}\left(x^{\prime}\right)=-\tilde{u}\left(\hat{x}^{\prime}\right) .
$$

On the other hand, because the gluing conditions that create $\tilde{M}$ must be consistent with the calibration and we glue $x$ to $\hat{x}^{\prime}$ and $x^{\prime}$ to $\hat{x}$, we have

$$
\tilde{u}(x)=\tilde{u}\left(\hat{x}^{\prime}\right), \quad \tilde{u}\left(x^{\prime}\right)=\tilde{u}(\hat{x}) .
$$

From the above equations we immediately get

$$
\tilde{u}(x)=-\tilde{u}\left(x^{\prime}\right) .
$$

Since $x$ and $x^{\prime}$ are the same point on the curve $\gamma_{R} \in M$, we have shown that the antisymmetric calibration $\tilde{u}$ in $\tilde{M}$ is an anti-continuous calibration on $M_{\text {cut }}^{1}$ across the curve $\gamma_{R}$. Thus we can work entirely on $M_{\text {cut }}^{1}$, supplementing the program (4.32) with the existence of a calibration satisfying, in addition to the usual constraints,

$$
\left.u\right|_{\gamma_{R}^{+}}=-\left.u\right|_{\gamma_{R}^{-}}
$$

where $\gamma_{R}^{ \pm}$are the two curves created by the cut along $\gamma_{R}$ (and similarly for $\gamma_{R^{c}}$ ).

In terms of the dual flows, the boundary condition at the cut effectively makes the cut into a source for a flow that eventually reaches the curve $\gamma$, solving the problem that in the original surface there could be no flux across $\gamma$. We also wish to emphasize that the construction of the double surface $\tilde{M}$ is not unique. If $R$, for example, was of genus two, we could produce the double by cutting either of two handles. We would expect that at the optimum either cut would yield calibrations that are equal on the band of saturating geodesics homotopic to $\gamma$, but away from these geodesics the two calibrations would be different, realizing the ambiguity pointed out below equation (4.39).

It is straightforward to see, by dualizing either on $\tilde{M}$ using the antisymmetry of $\tilde{u}$ or dualizing on $M$ using the boundary condition (8.20), that the corresponding dual scalar $\varphi$ is also anti-continous across the cut(s):

$$
\left.\varphi\right|_{\gamma_{R}^{+}}=-\left.\varphi\right|_{\gamma_{R}^{-}}
$$

This is also consistent with the relation (7.14) between the $u$ 's and the $\varphi$ 's. The dual objective will tend to make the values of $\varphi$ small at the sides of the cut. Indeed, if we consider a cycle orthogonal to $\gamma_{R}$ on the handle, the discontinuity at the cut requires an expensive gradient of $\varphi$ along that cycle. (Recall that $|d \varphi|$ enters negatively in the dual objective, which is being maximized.) The program will make the values of $\varphi$ small at the cut, almost as if the cuts were boundaries, where we know that $\varphi$ must vanish.

For each homotopy class in $S_{0}$ we must include in our programs a calibration $u$ or a scalar $\varphi$ subject to these anti-continuous boundary conditions across the cuts necessary to produce a double cover rendering the homotopy class nontrivial in homology.

\subsubsection{Analysis of curves on $\tilde{M}$}

Of course, our original intent was not to constrain members of the homology class $\tilde{C} \in H_{1}(\tilde{M})$ where $\tilde{\gamma}_{1}$ belongs, but rather members of the homotopy class $D \in \pi_{1}(M)$. Given that the metric on 
$\tilde{M}$ is obtained by lifting the metric on $M$ symmetrically, any curve on $\tilde{M}$ descends to a curve on $M$ with the same length. Let $C$ be the image under descent of $\tilde{C}$ :

$$
C:=\left\{m=p\left(m^{\prime}\right), m^{\prime} \in \tilde{C}\right\} .
$$

Each element $m$ of $C$ is a curve or a sum of curves on $M$; however, $C$ is a priori neither a homotopy nor a homology class. Since $\tilde{\gamma}_{1} \in \tilde{C}$ and $\tilde{\gamma}_{1}$ descends to $\gamma_{0}$, we have

$$
\gamma_{0} \in C \text {. }
$$

The calibration $\tilde{u}$ constrains the length of every element in $\tilde{C}$ and thus of every element in $C$. First we prove that every curve in the homotopy class $D$ of $\gamma_{0}$ is an element of $C$, showing we have succeeded in constraining all curves in $D$. Then we show that the constraints on any other elements of $C$ are not new.

Lemma 2. $C$ contains entire homotopy classes: given homotopic curves $\gamma, \gamma^{\prime}$ on $M$, if $\gamma \in C$ then $\gamma^{\prime} \in C$.

Proof: Since $\gamma \in C$ there exists an element $\tilde{\gamma}$ of $\tilde{C}$ that descends to $\gamma$. The homotopy deformation from $\gamma$ to $\gamma^{\prime}$ can be lifted to a homotopy on $\tilde{M}$ from $\tilde{\gamma}$ to a curve $\tilde{\gamma}^{\prime}$ that descends to $\gamma^{\prime}$. This fact follows from the "covering homotopy theorem" (see [35], Chapter 3, Theorem 3). Homotopic implies homologous, so $\tilde{\gamma}^{\prime}$ is in $\tilde{C}$, therefore $\gamma^{\prime}$ is in $C$.

Since $\gamma_{0} \in C$, the lemma shows that, as claimed, the full homotopy class $D \in S_{0}$ is contained in $C$.

We now argue that the constraint on all curves in $C$ does not impose constraints that go beyond those of the homotopy minimal-area problem. If a curve in $C$ is a sum of curves in which at least one is homotopically nontrivial, this is acceptable because we aim to constrain all homotopically nontrivial curves, even those not in $D$. We now show that none of the elements of $C$ are homotopically trivial curves, or sums of homotopically trivial curves. This follows from Lemma 1: if homotopically trivial curves were contained in $C$, all of them including the constant curve must be included in $C$. But a constant curve lifts to a constant curve on $\tilde{M}$, and sums of constant curves lift to sums of constant curves on $\tilde{M}$. But neither a constant curve nor sums of them are elements of $\tilde{C}$.

This concludes our proof that the calibration $\tilde{u}$ on $M$ will properly constrain the homotopy class $D$ without imposing unacceptable additional constraints.

\subsubsection{Final form of primal program}

We now give the form of the full convex program for the minimal-area homotopy problem for $M$. We assume that $S_{0}$ contains a set of homotopy classes $D_{0}^{k}$ with $k \in K_{0}$ (with $K_{0}$ a set), with Jordan representatives $\gamma_{k}$ that require cutting curves $\gamma_{R k}$ (with \pm labels for the resulting curves) and double covers to render them homologically nontrivial. We also have calibrations $u^{k}$ anti-continuous across 
the cutting curves $\gamma_{R k}$. The program is

$$
\begin{aligned}
& \text { Minimize } \int_{M} \sqrt{g^{0}} \Omega \text { over } \Omega \text { (function), } u^{\alpha}, u^{k} \text { (one-forms) } \\
& \text { subject to: } \quad\left|u^{\alpha}\right|_{0}^{2}-\Omega \leq 0 \text {, } \\
& d u^{\alpha}=0 \\
& 1-\int_{m_{\alpha}} u^{\alpha}=0, \forall \alpha \in J_{0}, \\
& \left|u^{k}\right|_{0}^{2}-\Omega \leq 0 \text {, } \\
& d u^{k}=0 \text {, } \\
& \left.u^{k}\right|_{\gamma_{R k}^{+}}+\left.u^{k}\right|_{\gamma_{\bar{R} k}^{-}}=0 \text {, } \\
& 1-\int_{\gamma_{k}} u^{k}=0, \quad \forall k \in K_{0} .
\end{aligned}
$$

An analogous expression could be written for the dual program involving the scalars $\varphi$ and the discontinuities $\nu$.

\section{Acknowledgments}

Barton Zwiebach would like to thank Larry Guth and Yevgeny Liokumovich for many instructive discussions on systolic geometry and on the challenges of proving existence of solutions. The work of M.H is supported by the National Science Foundation through Career Award No. PHY-1053842, by the U.S. Department of Energy under grant DE-SC0009987, and by the Simons Foundation through a Simons Fellowship in Theoretical Physics. The work of B.Z. is supported by the U.S. Department of Energy under grant Contract Number DE-SC0012567. M.H. would also like to thank MIT's Center for Theoretical Physics for hospitality and a stimulating research environment during his sabbatical year.

\section{A Notation and some useful formulae}

Consider a two-dimensional surface $M$ with a metric $g_{\mu \nu}$. For vectors $v_{1}, v_{2}$ on the surface the inner product $(\cdot, \cdot)$ and the norm $|\cdot|$ are defined by

$$
\left\langle v_{1}, v_{2}\right\rangle \equiv g_{\mu \nu} v_{1}^{\mu} v_{2}^{\nu}, \quad|v|^{2} \equiv\langle v, v\rangle
$$

For one-forms $u$, with components $u_{\mu}$ we take

$$
\left\langle u_{1}, u_{2}\right\rangle \equiv g^{\mu \nu} u_{1 \mu} u_{2 \nu}, \quad|u|^{2} \equiv\langle u, u\rangle
$$

Given a one-form $u$ with components $u_{\mu}$ we define the associated vector $\hat{u}$ with components

$$
\hat{u}^{\mu} \equiv g^{\mu \nu} u_{\nu} .
$$

A one form $u$ acting on a vector $v$ gives $u(v)$ with

$$
u(v)=u_{\mu} v^{\mu}=\langle\hat{u}, v\rangle .
$$


It follows also that

$$
|u|^{2}=|\hat{u}|^{2}=|u(\hat{u})|
$$

The Hodge dual $* u$ of a one-form $u$ is a one form with components

$$
(* u)_{\mu} \equiv u_{\rho} \varepsilon^{\rho \nu} g_{\nu \mu}, \quad \varepsilon^{\mu \nu} \equiv \frac{\epsilon^{\mu \nu}}{\sqrt{g}}, \quad \epsilon^{12}=1 .
$$

Additional useful relations are

$$
\epsilon^{\mu \nu}=g g^{\alpha \mu} g^{\beta \nu} \epsilon_{\alpha \beta}, \quad \epsilon_{12}=1 .
$$

The Hodge dual operation depends only on the Weyl class of the metric. This is clear because

$$
(* u)_{\mu} \equiv u_{\rho} \epsilon^{\rho \nu} \frac{g_{\nu \mu}}{\sqrt{g}}
$$

and the rightmost ratio is Weyl invariant. One can also check that

$$
(* u)_{\mu}=u_{\rho} \sqrt{g} g^{\rho \alpha} \epsilon_{\alpha \mu}, \quad * d x^{\mu}=\sqrt{g} g^{\mu \alpha} \epsilon_{\alpha \rho} d x^{\rho} .
$$

For a conformal metric one has $(* u)_{1}=-u_{2},(* u)_{2}=u_{1}$. In general, one has

$$
*(* u)=-u, \quad \text { and }\left\langle * u_{1}, * u_{2}\right\rangle=\left\langle u_{1}, u_{2}\right\rangle .
$$

It follows that the one forms $u$ and $* u$ are orthogonal

$$
\langle u, * u\rangle=0
$$

and so are the vectors associated to $u$ and $* u$. We define the volume form $\omega$

$$
\omega \equiv \sqrt{g} d^{2} x \equiv \sqrt{g} d x^{1} \wedge d x^{2} .
$$

We then have

$$
d x^{\mu} \wedge d x^{\nu}=\epsilon^{\mu \nu} d x^{1} \wedge d x^{2}=\epsilon^{\mu \nu} d^{2} x=\varepsilon^{\mu \nu} \sqrt{g} d^{2} x=\varepsilon^{\mu \nu} \omega .
$$

It is a simple calculation to verify that:

$$
u_{1} \wedge u_{2}=\left\langle * u_{1}, u_{2}\right\rangle \omega
$$

Given a closed one-form $u$, the vector $v$ associated with the dual form $* u$ is divergenceless:

$$
d u=0 \text { then } v \equiv \widehat{* u} \text { satisfies } \nabla_{\mu} v^{\mu}=0 \text {, and }|u|=|v| \text {. }
$$

These identities establish the equivalence of calibrations and flows.

For the metric $d s^{2}=E d x_{1}^{2}+G d x_{2}^{2}$ the Gaussian curvature $K(x)$ is given by

$$
K(x)=-\frac{1}{2 \sqrt{E G}}\left[\partial_{1}\left(\frac{\partial_{1} G}{\sqrt{E G}}\right)+\partial_{2}\left(\frac{\partial_{2} E}{\sqrt{E G}}\right)\right] .
$$

The scalar curvature $R$ is twice the Gaussian curvature: $R=2 K$. 
References

[1] E. Witten, "Noncommutative Geometry and String Field Theory," Nucl. Phys. B 268, 253 (1986). doi:10.1016/0550-3213(86)90155-0

\section{Please revise}

citations

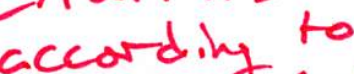


[19] H Federer, Real flat chains, cochains and variational problems, Indiana Univ. Math. J. 24 (1974/75) 351-407.

G. Strang, Maximal flow through a domain, Math. Programming 26 (1983), no. 2 123-143;

R. Nozawa, Max-flow min-cut theorem in an anisotropic network, Osaka J. Math. 27 (1990), no. 4 805-842;

J. M. Sullivan, A crystalline approximation theorem for hypersurfaces. ProQuest LLC, Ann Arbor, MI, 1990. Thesis (Ph.D.) Princeton University.

[20] M. Headrick and V. E. Hubeny, "Riemannian and Lorentzian flow-cut theorems," arXiv:1710.09516 [hep-th].

[21] M. Freedman and M. Headrick, "Bit threads and holographic entanglement," Commun. Math. Phys. 352, no. 1, 407 (2017) doi:10.1007/s00220-016-2796-3 [arXiv:1604.00354 [hep-th]].

[22] M. Headrick and B. Zwiebach, "Minimal-area metrics on the Swiss cross and punctured torus," arXiv:1806.00450 [hep-th].

[23] M. Headrick and B. Zwiebach, "String diagrams from minimal area metrics of non-positive curvature", in preparation.

[24] M. G. Katz and S. Sabourau, "Systolically extremal nonpositively curved surfaces are flat with finitely many singularities," to appear in Journal of Topology and Analysis. DOI: 10.1142/S1793525320500144 [arXiv:1904.00730]

[25] K. Ranganathan, "A Criterion for flatness in minimal area metrics that define string diagrams," Commun. Math. Phys. 146, 429 (1992). doi:10.1007/BF02097012

[26] M. Wolf and B. Zwiebach, "The Plumbing of minimal area surfaces," Journal of Geometry and Physics 15 (1994) 23-56. [hep-th/9202062].

[27] E. D'Hoker and S. B. Giddings, "Unitary of the Closed Bosonic Polyakov String," Nucl. Phys. B 291, 90 (1987). doi:10.1016/0550-3213(87)90466-4

[28] G. Zemba and B. Zwiebach, J. Math. Phys. 30, 2388 (1989). doi:10.1063/1.528569

[29] H. Sonoda and B. Zwiebach, "Closed String Field Theory Loops With Symmetric Factorizable Quadratic Differentials," Nucl. Phys. B 331, 592 (1990). doi:10.1016/0550-3213(90)90086-S

[30] S. F. Moosavian and R. Pius, "Hyperbolic Geometry and Closed Bosonic String Field Theory I: The String Vertices Via Hyperbolic Riemann Surfaces," arXiv:1706.07366 [hep-th].

[31] M. Berger, "A l'ombre de Loewner," Ann. Sci. Ecole Norm. Sup. Paris 5 (1972) 241-260.

[32] B. Zwiebach, Commun. Math. Phys. 141, 577 (1991). doi:10.1007/BF02102817

[33] R. Bott and L. Tu, Differential Forms in Algebraic Topology, Springer Verlag, New York (1982).

[34] N. J. Hicks, Notes on differential geometry, Van Nostrand (1965).

[35] I. M. Singer and J. A. Thorpe, Lecture notes on elementary topology and geometry, Undergraduate Texts in Mathematics, Springer-Verlag New York - Heidelberg - Berlin (1976).

[36] R. Harvey and B.H. Lawson, Jr., "Calibrated geometries", Acta Math., 148 (1982) 47-157.

[37] M. Sion, "On general minimax theorems", Pacific J. Math., 8 (1958), 171-176. 